\title{
Calcareous nannofossils from the upper Campanian-Maastrichtian (Upper Cretaceous) in the Kladorub Formation (Kula tectonic zone, NW Bulgaria)
}

\author{
Georgi Granchovski \\ Geological Institute, Bulgarian Academy of Sciences, Acad. G. Bonchev Street, Bl. 24, 1113 Sofia, Bulgaria; \\ e-mail: georgi2801@geology.bas.bg
}

(Accepted in revised form: April 2019)

\begin{abstract}
A detailed investigation into the calcareous nannofossils from the upper Campanian-Maastrichtian deposits of the Kladorub Formation (NW Bulgaria) has been carried out in order to examine their taxonomic content and test the applicability of cosmopolitan zonation schemes for this stratigraphic interval in the country. The Kladorub Formation is composed of silty to fine-sandy marlstones and rare marly limestones, occasionally interbedded with sandstone layers. The recovered nannofloras are abundant, taxonomically diverse and exhibit predominantly moderate preservation, which allowed precise taxonomic identifications and biostratigraphic analysis to be made. As a result, the presence of two previously undocumented, biostratigraphically significant taxa has been recorded (i.e., Eiffellithus parallelus and Ceratolithoides kamptneri). Consequently, the studied Upper Cretaceous sediments have been assigned to subzone UC15d ${ }^{\mathrm{TP}}$ (pars.)-subzone UC20d ${ }^{\mathrm{TP}}$; in the uppermost $2 \mathrm{~m}$ of the section, the presence of zone NP1 has also been indicated, which is in concordance with previous authors' data. Due to the lack of proper chronostratigraphic framework for the Kladorub Formation, top Uniplanarius trifidus and base Lithraphidites quadratus have, respectively, been used to approximate the Campanian/Maastrichtian and lower/upper Maastrichtian boundaries. The Cretaceous/Palaeogene boundary, however, could not be drawn with precision, because it falls within a 6.5-m interval of non-exposure. The resulting biostratigraphic framework offers higher stratigraphic resolution than previously used local zonation schemes and allows correlation with coeval sedimentary successions from other parts of the Tethyan and Boreal realms.
\end{abstract}

Granchovski, G. 2019. Calcareous nannofossils from the upper Campanian-Maastrichtian (Upper Cretaceous) in the Kladorub Formation (Kula tectonic zone, NW Bulgaria). Geologica Balcanica 48 (1), 73-101.

Keywords: calcareous nannofossils, biostratigraphy, upper Campanian-Maastrichtian, Kula tectonic zone, NW Bulgaria.

\section{INTRODUCTION}

The Upper Cretaceous Series in the Kula tectonic zone (NW Bulgaria; Fig. 1) has long posed a challenge to researchers due to the almost ubiquitous lack of exposures. To date, only a small number of limited outcrops are known, mainly nigh the town of Kula and in the vicinity of Lake Rabisha. Information about the distribution, lithology and stratigraphy of Upper Cretaceous strata has come primarily from subsurface data obtained through numerous exploratory oil-wells drilled between the 1960s and the 1980s. In these boreholes, however,
Upper Cretaceous deposits were drilled almost entirely without core recovery and, in addition, recovered microfossils were largely rare and too poorly preserved to allow precise bio- and/or chronostratigraphic subdivisions (e.g., Valkov, 1977; Monova and Ivanov, 1982; Peycheva and Parvanova, 1989). Thus, age assignments have often been based solely on lithologic comparisons with adjacent boreholes.

In current Bulgarian literature, the Upper Cretaceous in this part of the country is associated with Albian-Cenomanian clay-carbonate deposits transgressively overlain by 'Turonian-Senonian' thick, flysch-like successions (V. Tzankov et al., 1960, 


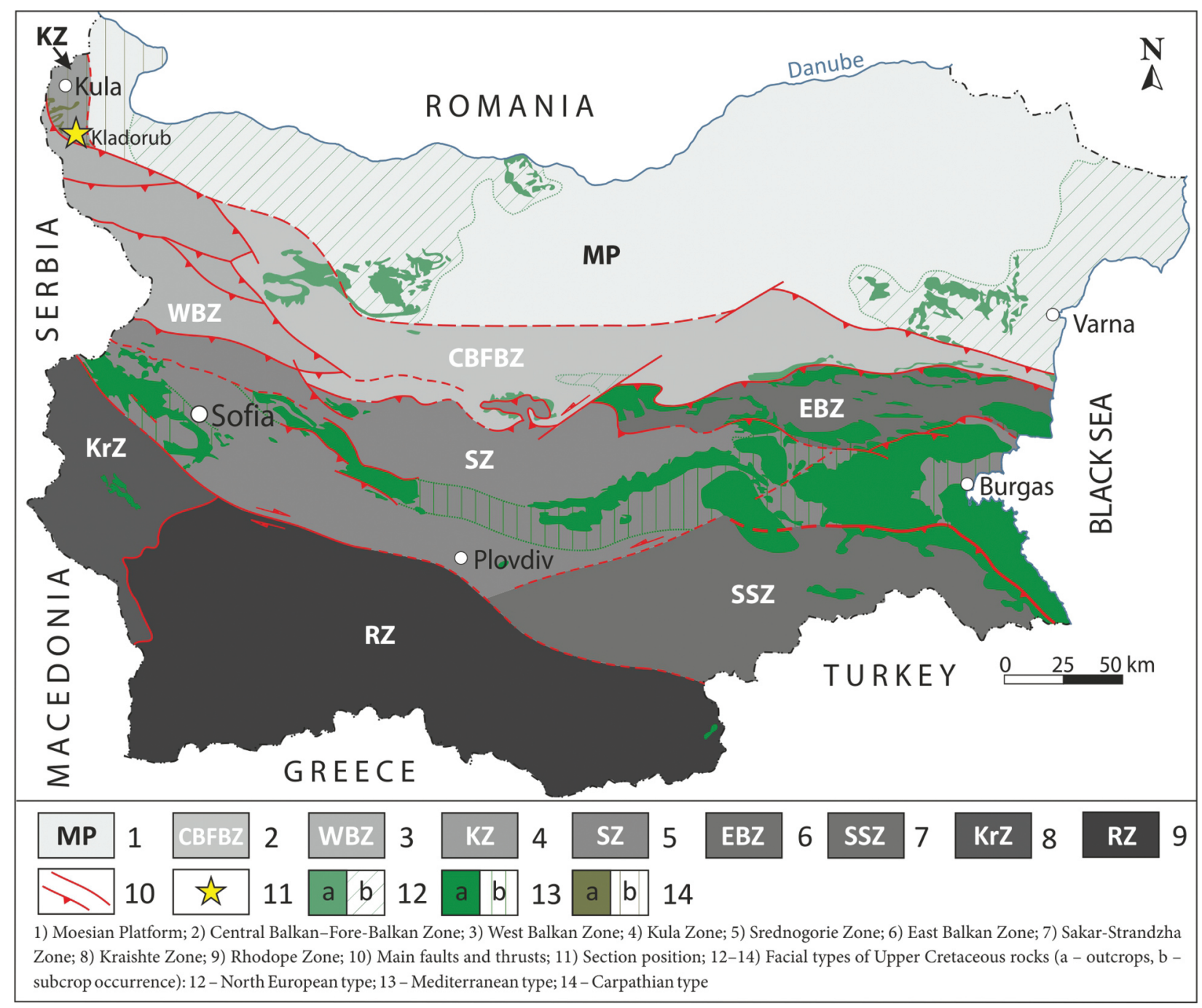

Fig. 1. Location of section Kladorub, shown on the tectonic map of Bulgaria (after Ivanov, 1983, 1998, 2017; simplified), with crop and subcrop occurrences of Upper Cretaceous rocks (after Dabovski et al., 2009).

1963; Tz. Tzankov, 1961, 1963a, b, 1964, 1972; Vrablyanski and Popov, 1962; Vaptzarova, 1963, 1965a, b, 1974, 1975a, b, 1976a, b, 1978; Tzankov, 1968; Nachev and Sultanov, 1980; Cheshitev et al., 1989; Nachev and Yanev, 1991; Decheva et al., 1995; Filipov et al., 1995; Haydoutov et al., 1995; Angelov and Dobrev, 2006a, b, c, d, e; Angelov et al., 2006; Dobrev, 2006; Dobrev and Angelov, 2006; Dabovski, 2009; Dabovski et al., 2009; Sinnyovsky, 2009), which laterally transition into Cenomanian-Danian limestone-marlstone sediments towards the Moesian Platform (Dabovski, 2009; Dabovski et al., 2009). V. Tzankov (1972) combined the Albian-Cenomanian clay-carbonate strata into his 'Rabiša Komplex' (formalized as the Rabisha Formation by Nikolov and Ruskova, 1993) and described the overlying flysch-like sediments as
'Kula Komplex' (formalized as the Kula Formation by Filipov et al., 1995). Additionally, he introduced a third complex ('Kladorub Komplex', later formalized as the Kladorub Formation by Filipov et al., 1995) encompassing a 200-m thick, Maastrichtian in age, succession of grey to grey-greenish silty marlstones exposed in the vicinity of Kladorub Village (Vidin District). Tz. Tzankov (1972) based his age assignments on previously published rare finds of ammonites, inoceramid bivalves and planktonic foraminifera (V. Tzankov et al., 1960; Tz. Tzankov, 1961, 1964, 1972; Vrablyanski and Popov, 1962; V. Tzankov, 1968). However, more recent studies, based on calcareous nannofossils and planktonic foraminifera, have shown that the stratigraphic extent of the Rabisha Formation reaches up to at least the uppermost Turonian (Granchovski, 2013), where- 
as the Kladorub Formation embraces the interval from the upper Campanian to the lower Eocene (Sinnyovsky and Petrov, 2000; Sinnyovsky et al., 2002; Sinnyovsky, 2003, 2004, 2007, 2013a, 2015; Stoykova and Ivanov, 2005; Stoykova et al., 2005, 2006; Valchev and Juranov, 2006; Stoykova, 2007). Furthermore, as heeded by Granchovski (2015), the definitions of some of the Upper Cretaceous stage boundaries in the region are outdated and imprecise. Thus, it is apparent that new investigations are needed to better understand the stratigraphy of the Upper Cretaceous sediments in NW Bulgaria.

In the last two decades, several papers involving or dedicated to Upper Cretaceous calcareous nannofossil biostratigraphy in the Kula Zone have emerged (Harkovska et al., 1999; Sinnyovsky and Petrov, 2000; Sinnyovsky et al., 2002; Sinnyovsky, 2003, 2004, 2007, 2011, 2013a, b, 2015; Stoykova, 2007; Stoykova et al., 2010; Granchovski, 2012, 2013, 2015, 2017; Sinnyovsky and Pavlishina, 2014). However, the majority of these (i.e., Sinnyovsky and Petrov, 2000; Sinnyovsky et al., 2002; Sinnyovsky, 2003, 2004, 2007, 2011, 2013a, b, 2015; Sinnyovsky and Pavlishina, 2014) rely on local zonation schemes, which are often based on obsolete/questionable taxonomic identifications. For instance, Sinnyovsky and Pavlishina (2014) used base Prediscosphaera cretacea to define the Albian/Cenomanian boundary in a surface exposure of the Rabisha Formation near Tolovishko Vrelo karst spring, but the two specimens they illustrated (Sinnyovsky and Pavlishina, 2014, Fig. 3k, l) are small, circular to subcircular, with narrow inner-rim cycle, which are actually characteristic features of Prediscosphaera columnata. The problem with using these local zonations is further exacerbated by the fact that they do not employ qualitative abundances of taxa, which can lead to discrepancies in biostratigraphic correlation, especially close to extinction events (see Thibault, 2016b).

The present paper is an account on the upper Campanian-Maastrichtian calcareous nannofossils from the type section for the Kladorub Formation (Figs 1-3). The aim of this study is to examine in detail their taxonomic diversity and to assess the applicability of cosmopolitan nannofossil zonations, namely Burnett’s (1998) UC scheme.

\section{GEOLOGICAL BACKGROUND AND SECTION}

In the sense of current geodynamic models, the sediments of the Kladorub Formation are assumed to have been deposited in the upper bathyal zone
(Stoykova et al., 2006) on the continental slope of a deep-water back-arc basin in the western periphery of the Moesian Platform (Dabovski, 2009; Dabovski et al., 2009). In terms of tectonics, its distribution is confined to the limits of Ivanov's (1983, 1998, 2017) Kula Zone (equivalent to the Kula tectonic unit of Dabovski and Zagorchev, 2009; Fig. 1). This para-autochtonous unit is regarded as a part of the South Carpathian orogenic system (Dabovski and Zagorchev, 2009).

Lithologically, the Kladorub Formation has been described as composed of monotonous grey to greygreenish indistinctly-bedded clayey-calcareous siltstones, interbedded with a limited number of silty marlstones (Sinnyovsky et al., 2002). Their age has been determined as late Campanian (pars.)Ypresian (pars.) (Sinnyovsky and Petrov, 2000; Sinnyovsky et al., 2002; Sinnyovsky, 2003, 2004, 2007, 2013a, 2015; Stoykova and Ivanov, 2005; Stoykova et al., 2005, 2006; Valchev and Juranov, 2006; Stoykova, 2007). Sinnyovsky (2004) claimed that the Kladorub Formation contains the most complete succession across the Cretaceous/Palaeogene boundary (KPgB) in Bulgaria. The KPgB itself is marked by a 2-cm dark layer classified as a clayeycalcareous siltstone (Sinnyovsky et al., 2002). In the upper parts of the unit, the Palaeocene-Eocene Thermal Maximum has been recognized (Stoykova and Ivanov, 2005; Stoykova et al., 2005, 2006).

The fossil content of the Kladorub Formation includes ammonites (rare - V. Tzankov et al., 1960; Tz. Tzankov, 1963, 1964b, 1972), inoceramid bivalves (rare - V. Tzankov et al., 1960; Vrablyanski and Popov, 1962; Tz. Tzankov, 1972), foraminifera (V. Tzankov et al., 1960; Vrablyanski and Popov, 1962; Valchev and Juranov, 2006) and calcareous nannoplankton (Sinnyovsky and Petrov, 2000; Sinnyovsky et al., 2000; Sinnyovsky, 2003, 2004, 2007, 2013, 2015; Stoykova and Ivanov, 2005; Stoykova et al., 2005, 2006; this study).

So far, the lower boundary of the Kladorub Formation has not been observed; in surface exposures, it is tectonic, with the granodiorites of the Belogradchik pluton (Tz. Tzankov, 1972; Filipov et al., 1995; Sinnyovsky and Petrov, 2000; Sinnyovsky et al., 2000; Sinnyovsky, 2003, 2004, 2007, 2009, 2013a, b, 2015; Angelov and Dobrev, 2006c; this study - Fig. 3). Tz. Tzankov (1972) opined that this lithostratigraphic unit overlies the flysch-like deposits of the Kula Formation and, in some areas of their distributions, the two units may even have lateral relations. The upper boundary is transgressive, with the sandstones and conglomerates of the Staropatitsa Formation (Tz. Tzankov, 1972; Filipov et al., 1995; Sinnyovsky and Petrov, 2000; Sinnyov- 

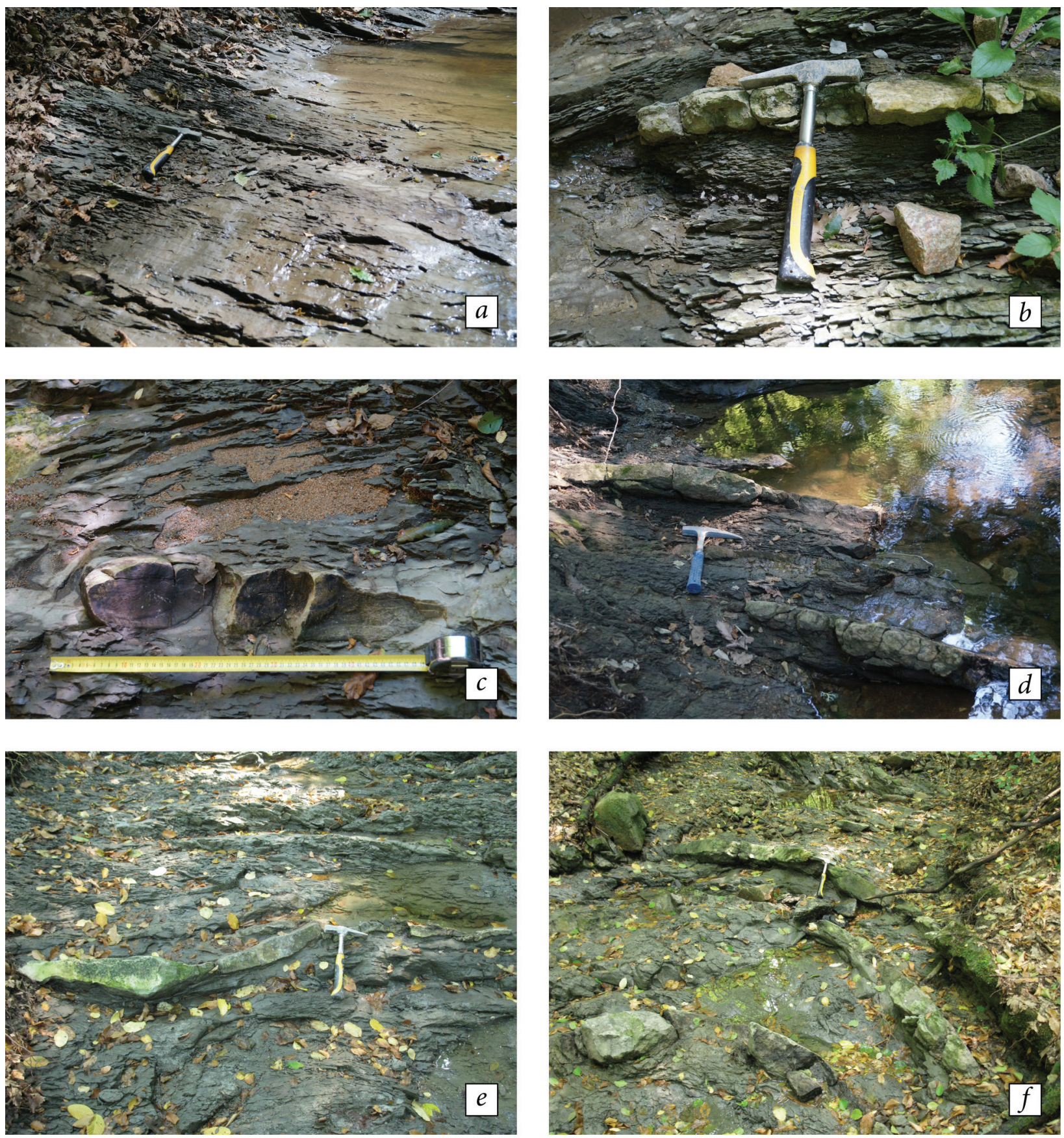

Fig. 2. Sediments from the type section for the Kladorub Formation. The stratigraphic succession is overturned, so upper portions of all images correspond to older strata. $a$ ) Thin-bedded grey to grey-greenish silty marlstones; $b$ ) sandstone layer sandwiched between laminated silty to fine-sandy marlstones; $c$ ) limestone layer with nodules; $d$ ) cleaved marlstones bracketed by sandstone (above) and marly limestone (below) layers; $e$ ) deformed sandstone layer at the base of a slump; $f$ ) slump structure.

sky et al., 2000; Sinnyovsky, 2003, 2004, 2007, 2009, 2013a, 2015; Angelov and Dobrev, 2006c; Stoykova, 2007) or with the Rakevtsi wedge of the Krivodol Formation (Stoykova, 2007).

Of all Upper Cretaceous lithostratigraphic units, the Kladorub Formation has the most limited distribution. It has not been recognized in borehole sec- tions. According to Tz. Tzankov (1972), it crops out only to the south and southwest of Kladorub Village, Vidin District. Stoykova (2007) mentioned an exposure near Rakovitsa Village, Vidin District, but described the sediments as tectonically overprinted and reported only low in abundance, poorly preserved nannofossil assemblages. It is assumed 


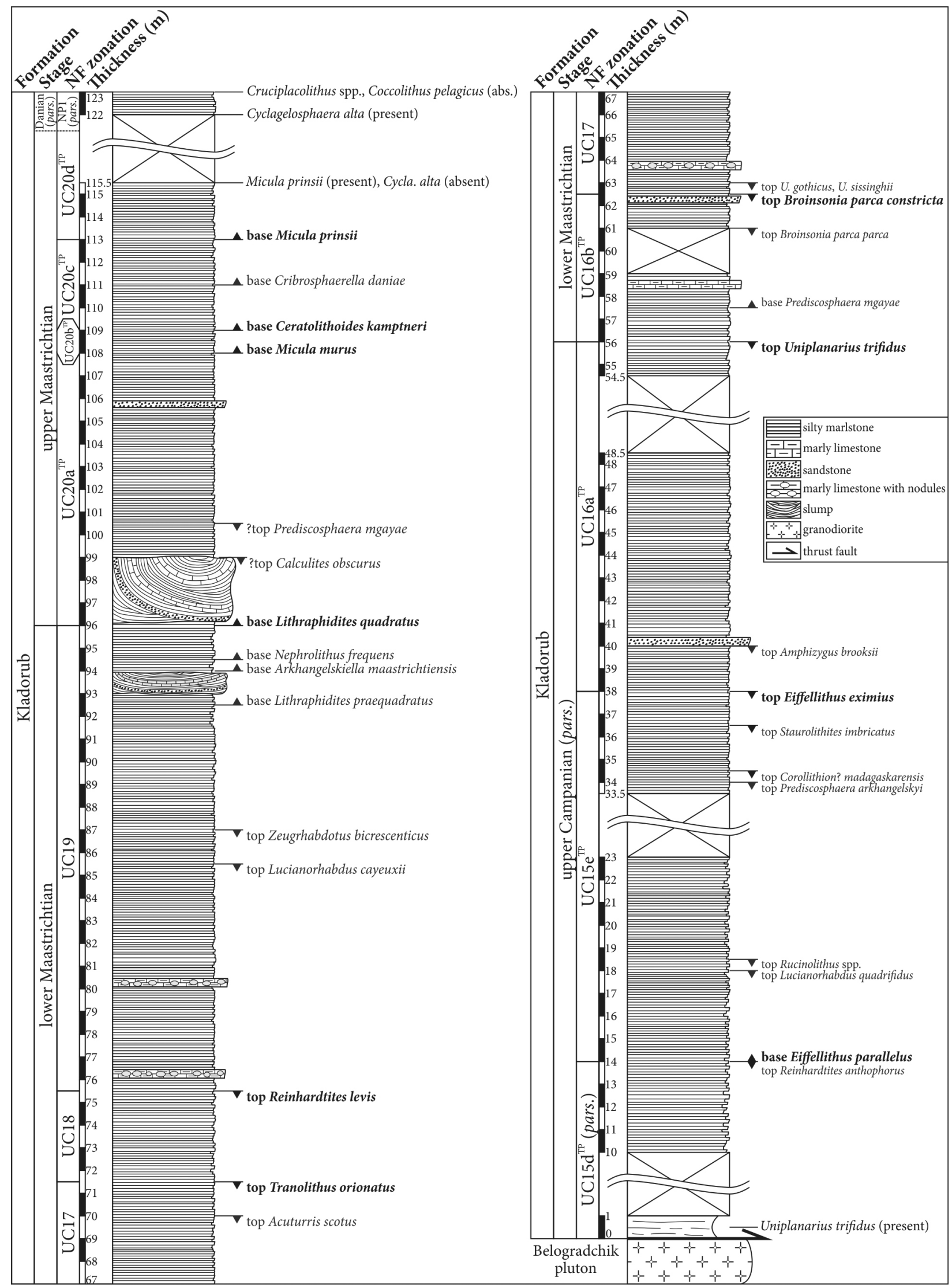

Fig. 3. Lithostratigraphy, lithology, calcareous nannofossil biostratigraphy and nannofossil events from section Kladorub. Lithostratigraphy after Filipov et al. (1995) and Angelov and Dobrev (2006c); biostratigraphy follows Burnett (1998) and Thibault (2016a). 
that the sediments of the Kladorub Formation are also developed to the east and northeast of the Kula Zone within the western parts of the Moesian Platform, where sedimentary rocks with the same lithology and analogous age have been penetrated by numerous boreholes (Stoykova, 2007).

In the present study, the stratotype section for the Kladorub Formation was chosen for investigation because: (1) it presents the most complete, continuous succession of the unit; (2) it is the leastaffected by tectonics exposure; (3) nannofossil assemblages are reportedly abundant, taxonomically rich and well preserved. It is located approximately $2.5 \mathrm{~km}$ to the southeast of Kladorub Village (Fig. 1; N43 $42^{\prime} 30^{\prime \prime}$; E22 $39^{\prime} 44^{\prime \prime}$ ) and crops out in the valley of a southern tributary of the Archar River. Here, to the southwest of the exposure, the granodiorites of the Belogradchik pluton are thrust over the Kladorub Formation. The stratigraphic succession is tectonically overturned (Sinnyovsky and Petrov, 2000; Sinnyovsky et al., 2000; Sinnyovsky, 2003, 2004, 2007, 2009, 2013a, 2015; Stoykova, 2007; this study). Sedimentary strata are exposed almost exclusively only in the riverbed, dipping to the SSW at a low angle $\left(15^{\circ}-45^{\circ}\right.$; in some limited intervals, usually in proximity to slumps, they become steeply inclined to subvertical). For the purposes of the present study, only the lowermost $123 \mathrm{~m}$ of the succession were investigated.

The Kladorub Formation is predominantly represented by grey to grey-greenish silty to finesandy marlstones and rare marly limestones and sandstones (Figs 2, 3). The marlstones are moderately hard, often crumbly. They are mostly indistinctly to very fine- or medium-bedded $(2-3 \mathrm{~cm}$ to 15-20 cm), laminated. When weathered, they become light grey. The limestones are hard, compact. They occur as infrequent light-grey beds $(8-9 \mathrm{~cm}$ to $15-16 \mathrm{~cm}$ ) or, occasionally, as lenses amongst the marlstones. Some of them contain nodules. Sandstones are also rare. They are grey-beige (rusty-grey when altered) and, with the exception of one 22-cm layer $40 \mathrm{~m}$ above the tectonic contact with the Belogradchik pluton, their thicknesses vary narrowly from $3-4 \mathrm{~cm}$ to $10-11 \mathrm{~cm}$. Interestingly, slump structures, which have not been mentioned by previous authors, were commonly observed. They occur in the upper half of the studied succession and range from $1 \mathrm{~m}$ to $3 \mathrm{~m}$ in thickness.

\section{MATERIAL AND METHODS}

A total of 164 samples, plus one test sample, have been investigated for their nannofossil content.
They were taken almost exclusively with 0.5 -m resolution; in a limited interval in the lower parts of the section where exposure was poor, sampling density was decreased to one sample per $1 \mathrm{~m}$. A distinct, 22-cm thick, resistant to weathering sandstone layer in the lower half of the section was used as a reference point and was conditionally numbered as 100; all sample numbers reflect their position above or below its lower surface in metres.

Calcareous nannofossil assemblages were examined in simple smear-slides, made following the methodology described by Bown and Young (1998). They were viewed at $1250 \times$ magnification, using an oil-immersion objective lens $(\times 100)$ on a Zeiss Axioskop 40 transmitting light-microscope. Micrographs were taken with a ProgRes GT3 digital camera in both cross-polarized light (with and without gypsum plate) and plane-polarized light. All images were uniformly enlarged at 2000× magnification in Photoshop and their contrast was increased by $10-15 \%$. Thereafter, they were sharpened, using the following technique: (1) a copy of the background layer, containing the original image, was made; (2) the copy layer was desaturated, lest original colours be altered, and then a High Pass filter was applied to it with a radius of 3-5 pixels; (3) finally, the blending mode of the copy layer was set to Soft Light, its opacity was lowered to $50-60 \%$ and all layers were merged into one. All operations in Photoshop were performed on lossless file formats (i.e., TIFFs) in order to prevent loss of image quality.

Relative abundances of the species were estimated semi-quantitatively over four traverses (three central and one random to avoid overlooking rarer taxa) for each slide and the data are presented as a range-chart (see Table A1 in the Appendix). On the chart, abundances are indicated as follows: $\mathrm{C}=$ common $(1-10$ specimens/field of view); $\mathrm{F}=$ frequent (one specimen/2-50 fields of view); $\mathrm{R}=$ rare (one specimen/51-100 fields of view); VR = very rare (one specimen/ traverse); $\mathrm{P}=$ present (one specimen/sample); and questionable (? = identification uncertain). Species occurrences interpreted as a result of reworking are indicated with an asterisk (*).

A qualitative estimate of calcareous nannofossil preservation was also made for each sample. It is indicated on the range-chart in Table A1 as: moderate $(\mathrm{M}=$ virtually all specimens are identifiable, although the appearance of certain taxa and/or features has been modified by secondary calcite dissolution and/or overgrowth); poor ( $\mathrm{P}=$ depleted assemblage due to calcite dissolution and/or taxonomic identification of a considerable portion of specimens is thwarted by calcite dissolution or secondary over- 
growth); very poor ( $\mathrm{VP}=$ much of the assemblage is dissolved and/or the majority of specimens are difficult or even impossible to identify). Most of the investigated samples are characterized by moderate preservation.

Taxonomic concepts follow those of PerchNielsen (1985) and Burnett (1998), although some more recent works have also been taken into account (Lees and Bown, 2005; Lees, 2007; Shamrock and Watkins, 2009; Thibault, 2010a, b). Full taxonomic descriptions, with authorship, of all taxa mentioned in the text and figures can be found online on Nannotax3 at http://www.mikrotax.org/Nannotax3/index.php?dir=Mesozoic. Burnett's (1998) UC zonation scheme, as supplemented by Thibault (2016a), was applied for biostratigraphic subdivision of the studied sedimentary succession.

Sample material and slides are housed at the Department of Palaeontology, Stratigraphy and Sedimentology of the Geological Institute "Str. Dimitrov” (Bulgarian Academy of Sciences).

\section{RESULTS}

A total of 137 species have been identified. Nannofossil preservation varies from very poor to moderate, the latter state being predominant. Small coccoliths (e.g., Biscutum constans, Corollithion? madagaskarensis, Prediscosphaera stoveri and small Staurolithites and Zeugrhabdotus species) and holococcoliths (Calculites, Lucianorhabdus, Octolithus and Russelia, as well as Lanternithus in the lowermost Palaeogene) are reasonably well and consistently represented in samples and, in comparison to other species, no excessive concentration of solution-resistant taxa (i.e., Watznaueria barnesiae and Micula staurophora) was observed. This suggests that, altogether, diagenesis has probably not impacted too deleteriously the assemblages. Persample species richness varies between 54 and 97 but most often stays between 70 and 80 . Significant and some rarely or never previously reported from the Upper Cretaceous of Bulgaria taxa are illustrated in Figs 4-18. Table A1 shows the semi-quantitative calcareous nannofossil data; the low-latitude part of Burnett's (1998) UC zonation scheme (UC ${ }^{\mathrm{TP}}$ ), as supplemented by Thibault (2016a) for zone UC16, has been applied to this. On Table A1, stratigraphical ranges of biostratigraphically significant taxa are highlighted, and a summary of the bioevents detected is provided on the right-hand side.

The calcareous nannofossil assemblages are dominated by Prediscosphaera cretacea (Fig. 10w, x), Watznaueria barnesiae (Fig. 12j), Micula stauro- phora (Figs 16h, 17k-m), Cribrosphaerella ehrenbergii (Fig. 9j, k) and Biscutum constans (Figs 9y, 10a). Of these, Prediscosphaera cretacea is ubiquitously the most abundant (when common, it is represented by 5-8 specimens/field of view, and its abundance never drops below one specimen/2 fields of view), whereas Watznaueria barnesiae (1-3 specimens/field of view to one specimen/2-6 fields of view) and Micula staurophora (1-2 specimens/ field of view to 1 specimen/2-12 fields of view) have somewhat subordinate presence, in comparison. Prediscosphaera microrhabdulina (Fig. 11h, $i, k$ ) and Prediscosphaera stoveri (Fig. $11 m, n$ ) are also considerable constituents of the assemblages throughout the studied succession.

The lowermost $10 \mathrm{~m}$ of the section proved to be somewhat problematic. Tectonic overprint of the sediments, coupled with very poor exposure, hindered sampling of this interval. At $\sim 2 \mathrm{~m}$ above the tectonic boundary with the granodiorites, one test sample was taken from indistinctly-bedded, poorly exposed, fragmented marls. The recovered nannofossils were poorly preserved, with low species richness (29 species). Despite that, reliably identifiable specimens of Uniplanarius trifidus and Eiffellithus eximius were observed. These two taxa, in the absence of Eiffellithus parallelus, indicate subzone $\mathrm{UC}_{15} \mathrm{~d}^{\mathrm{TP}}$, which is consistent with data from the $4 \mathrm{~m}$ immediately above the covered interval. However, these marls are herein considered subcrop occurrence and, thus, the test sample is not recorded in Table A1.

The first reliably identifiable specimens of Eiffellithus parallelus (Fig. $7 j-O$ ) were noted in sample Kl-74 (at $14 \mathrm{~m}$ ), which draws the boundary between subzones $\mathrm{UC} 15 \mathrm{~d}^{\mathrm{TP}}$ and $\mathrm{UC} 15 \mathrm{e}^{\mathrm{TP}}$. The last consistently occurring specimens of Reinhardtites anthophorus (Fig. $5 c-g$ ) were observed in the same sample; up-section, this species was present in only one sample, Kl-77, very rare in abundance. According to Burnett (1998), top Reinhardtites anthophorus indeed falls within UC15e ${ }^{\mathrm{TP}}$ at low palaeolatitudes. The consecutive tops of Lucianorhabdus quadrifidus (at $18 \mathrm{~m}$; Fig. 14b, c), Rucinolithus spp. (at $18.5 \mathrm{~m}$ ), Prediscosphaera arkhangelskyi (at $34 \mathrm{~m}$; Fig. 10u, v), Corollithion? madagaskarensis (at $34.5 \mathrm{~m}$; Fig. $8 r-u$ ) and Staurolithites imbricatus (at $36.5 \mathrm{~m}$; Fig. $5 p, q$ ) were also recorded within subzone UC15 $\mathrm{e}^{\mathrm{TP}}$.

The last consistent occurrence of Eiffellithus eximius (Fig. $7 c-g$ ) was detected in sample Kl-98 (at $38 \mathrm{~m}$ ). Up-section, this taxon is present very sporadically (observed in only eight samples at $40 \mathrm{~m}$, $43.5 \mathrm{~m}, 59 \mathrm{~m}, 89 \mathrm{~m}, 108 \mathrm{~m}, 110.5 \mathrm{~m}, 112 \mathrm{~m}, 112.5 \mathrm{~m}$ and $123 \mathrm{~m}$ ), generally very low in abundance and 

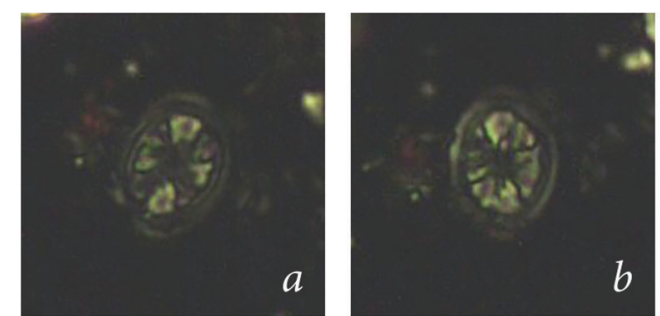

Ahmuellerella alboradiata

$$
\text { Kl-96.5 }
$$

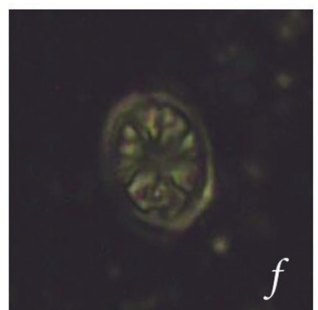

Ahmuellerella regularis Kl-175 (XPL)

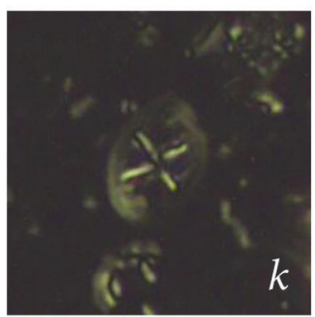

Chiastozygus bifarius K1-77.5 (XPL)

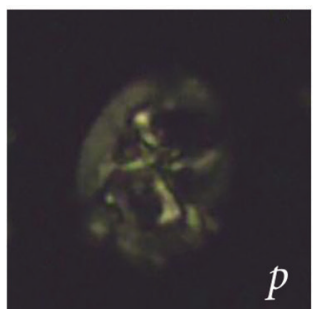

Chiastozygus litterarius Kl-93.5 (XPL)

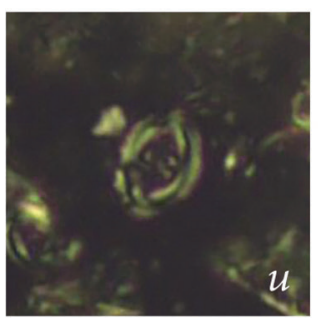

Placozygus fibuliformis Kl-170 (XPL)
(XPL)

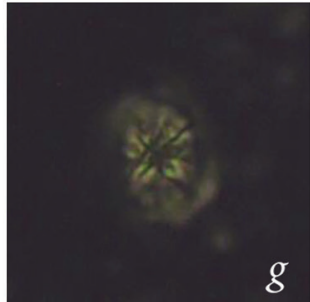

(XPL)

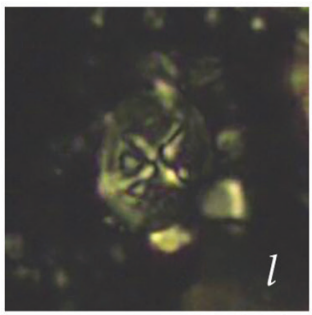

Chiastozygus bifarius Kl-151.5 (XPL)

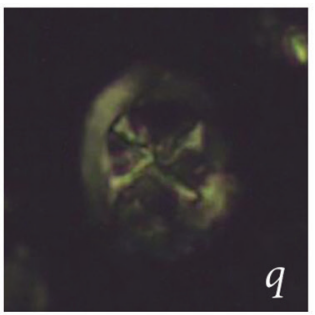

(XPL)

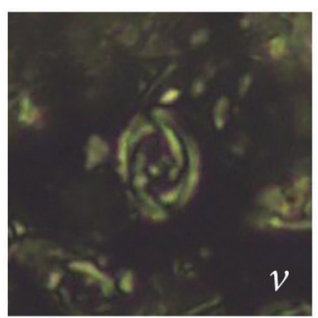

(XPL)

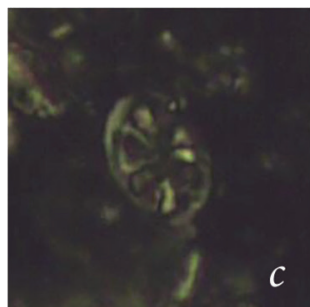

Ahm. octoradiata Kl-152.5

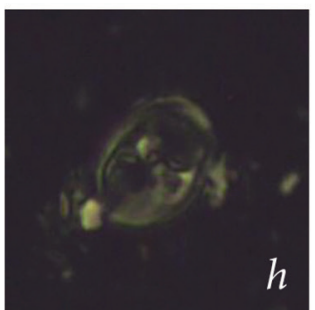

Amphizygus brooksii Kl-71 (XPL)

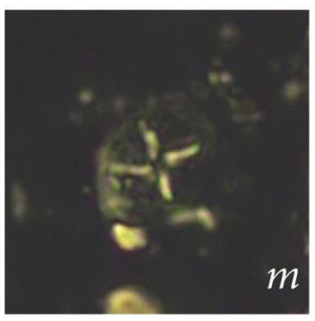

(XPL)

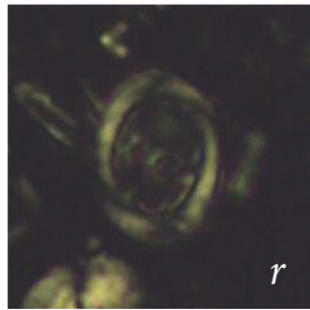

Loxolithus armilla Kl-76 (XPL)

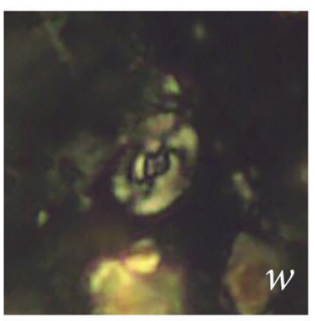

Placozygus spiralis Kl-175 (XPL)

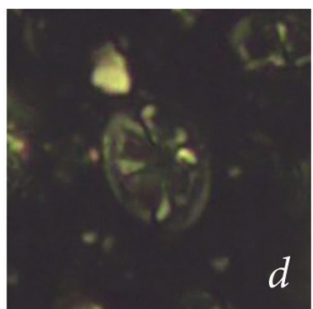

Ahm. octoradiata Kl-170

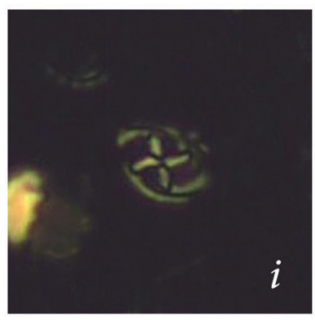

Chiastozygus antiquus Kl-175 (XPL)

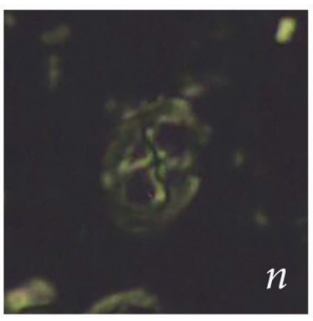

Chiastozygus litterarius Kl-73 (XPL)

(XPL)

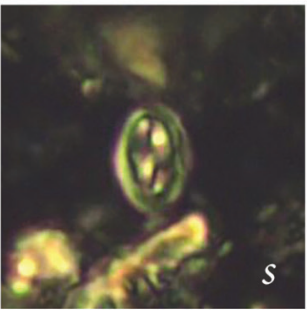

Neocrepidolithus dirimosus

Kl-183 (XPL)

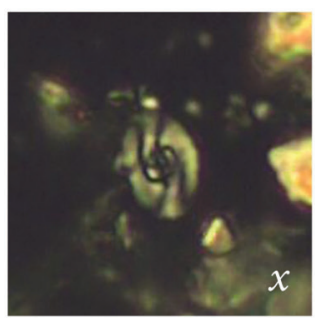

(XPL)

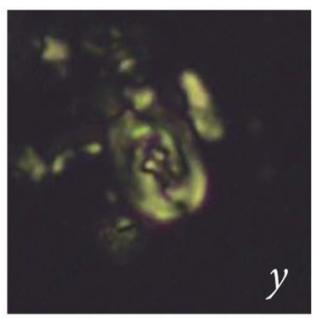

Placozygus spiralis Kl-175.5 (XPL)

Ahm. octoradiata Kl-170.5

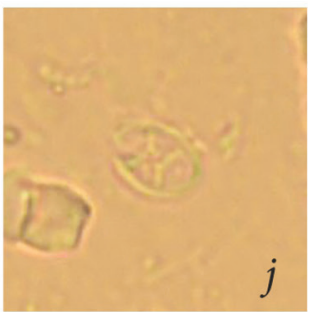

(PPL)
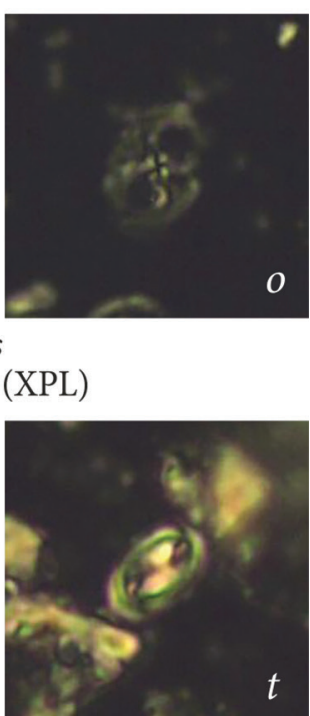

(XPL)

\section{$5 \mu \mathrm{m}$}

Fig. 4. Calcareous nannofossils from section Kladorub (Chiastozygaceae). Scale bar applies to all images. Abbreviations: XPL cross-polarized light; PPL - plane-polarized light. 


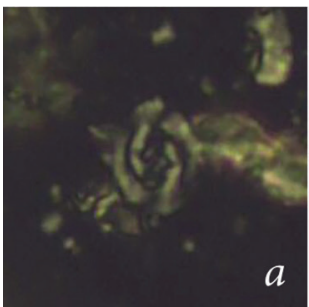

Placozygus spiralis Kl-119 (XPL)

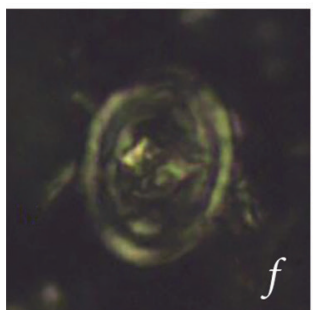

Reinhardtites anthophorus

Kl-72 (XPL)

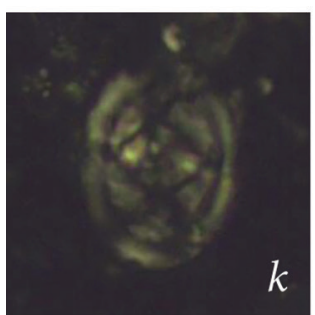

Reinhardtites levis

Kl-76 (XPL)

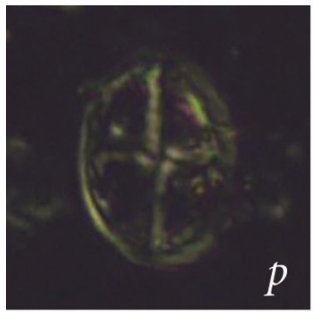

Staurolithites imbricatus

Kl-77.5 (XPL)

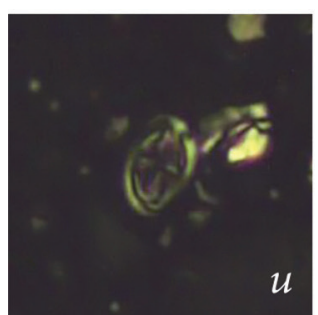

Staurolithites laffittei Kl-71 (XPL)

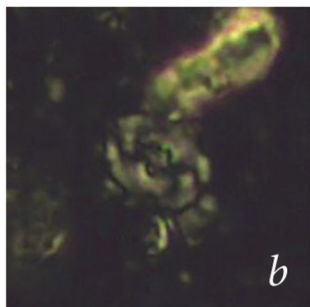

(XPL)

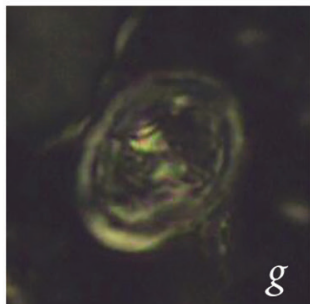

(XPL)

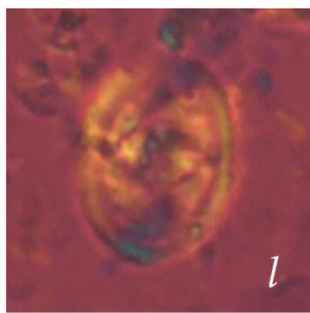

(GP)

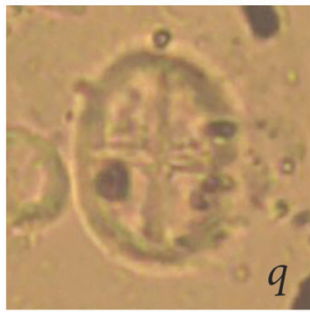

(PPL)

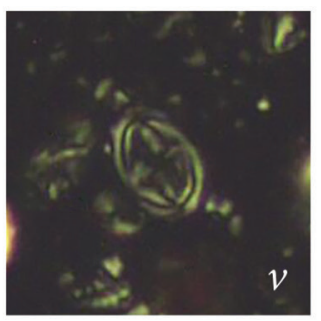

Staurolithites laffittei Kl-77 (XPL)
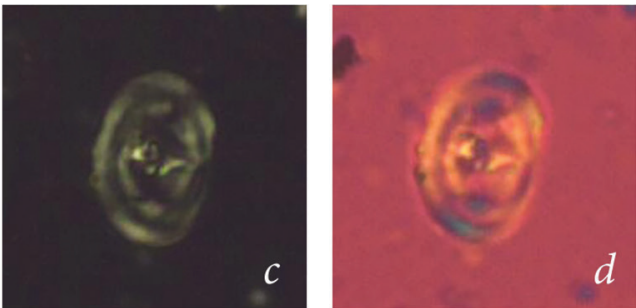

Reinhardtites anthophorus

Kl-71 (XPL)

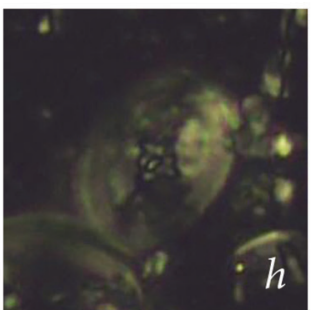

Reinhardtites levis Kl-73 (XPL)

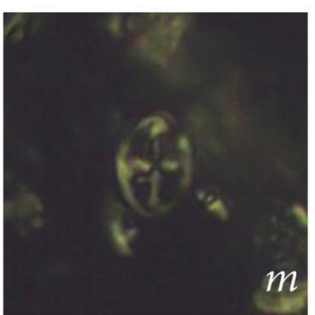

Staurolithites flavus Kl-96.5 (XPL)

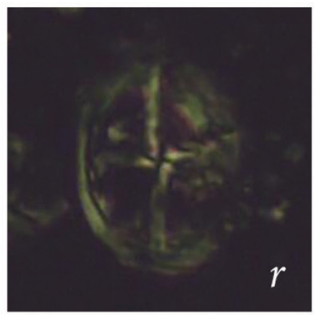

(XPL)

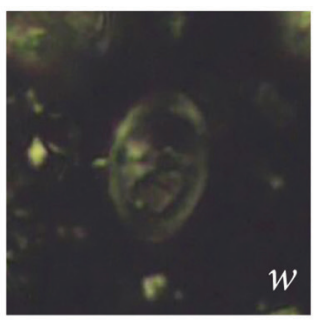

Tranolithus orionatus Kl-73 (XPL)
(GP)

(PPL)

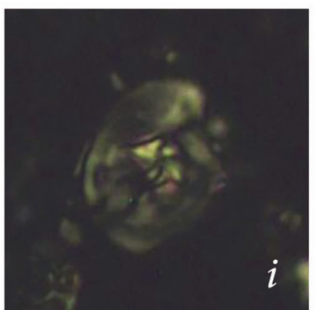

Reinhardtites levis Kl-73 (XPL)

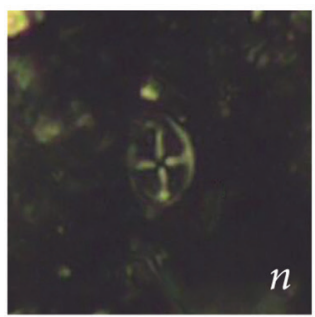

(GP)

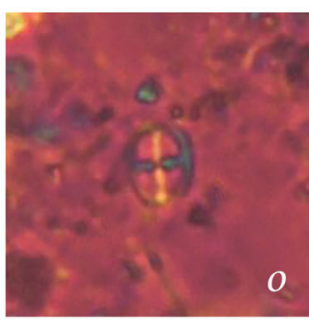

Staurolithites flavus Kl-169 (XPL)

(GP)
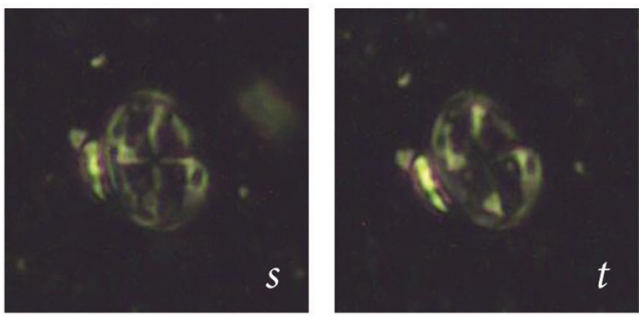

Staurolithites mielnicensis Kl-71 (XPL)

(XPL)
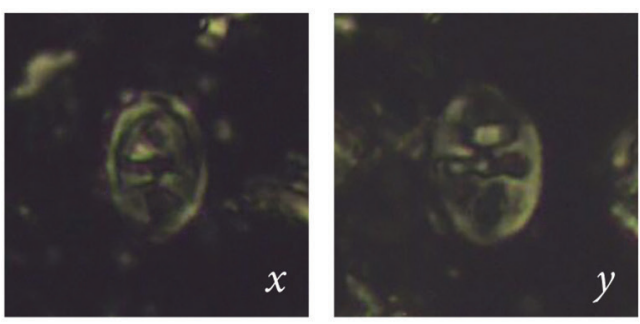
$\begin{array}{ll}\text { Tranolithus orionatus } & \text { Tranolithus orionatus } \\ \text { Kl-77 (XPL) } & \text { Kl-119 (XPL) }\end{array}$

\section{$5 \mu \mathrm{m}$}

Fig. 5. Calcareous nannofossils from section Kladorub (Chiastozygaceae). Scale bar applies to all images. Abbreviations: XPL cross-polarized light; PPL - plane-polarized light; GP - gypsum plate. 


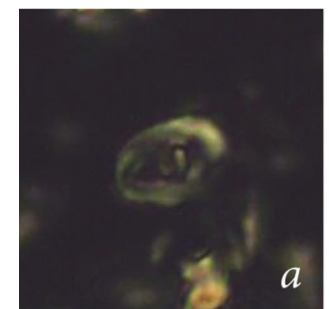

\section{Z. bicrescenticus}

Kl-72 (XPL)

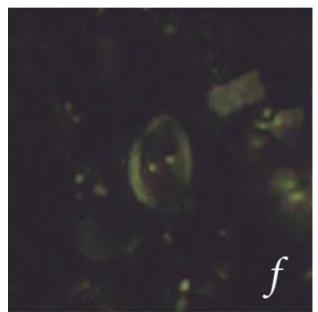

Zeugrhabdotus erectus Kl-175 (XPL)

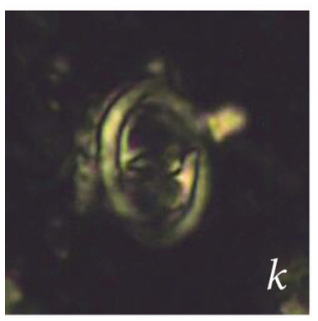

Zeugrhabdotus praesigmoides

K1-72 (XPL)

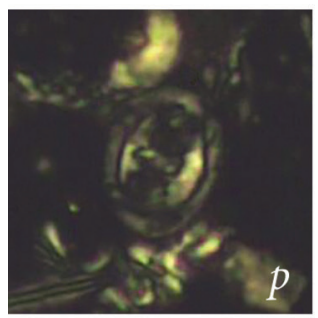

Zeugrhabdotus praesigmoides

K1-168.5 (XPL)

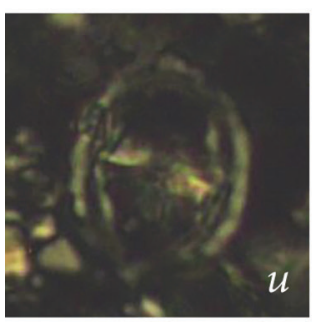

Zeugrhabdotus sigmoides

Kl-155.5 (XPL)

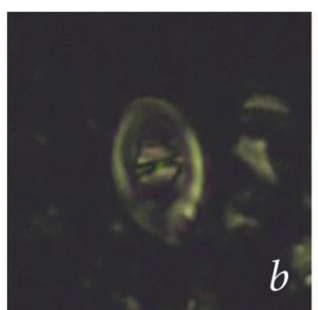

Z. bicrescenticus Kl-73 (XPL)

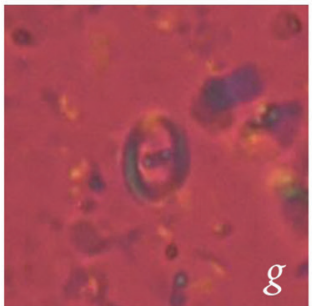

(GP)

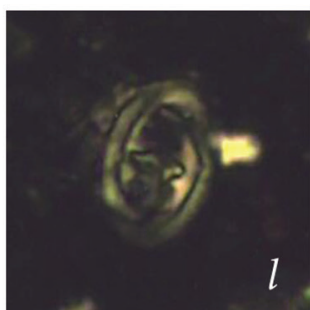

(XPL)

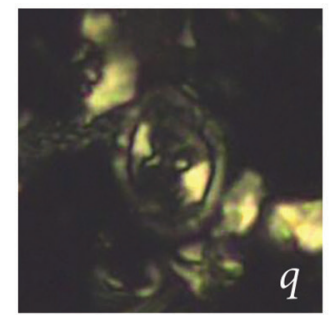

(XPL)

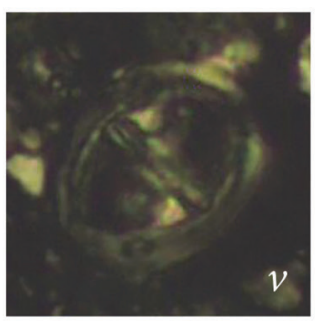

(XPL)

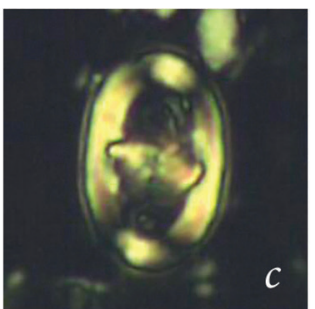

Z. embergeri

Kl-70 (XPL)

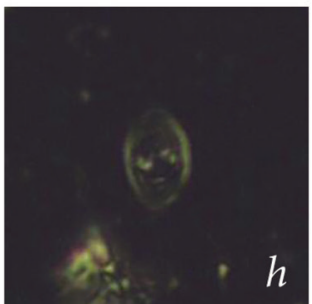

Zeugrhabdotus erectus Kl-175 (XPL)

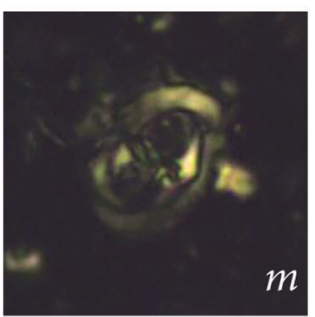

$m$
(XPL)

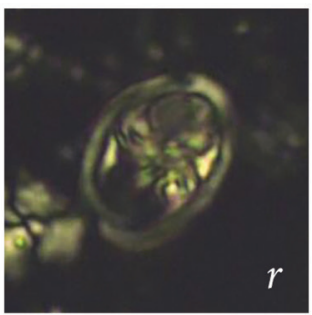

Zeugrhabdotus sigmoides

Kl-73 (XPL) (GP)

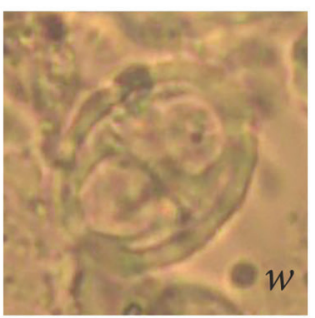

(PPL)

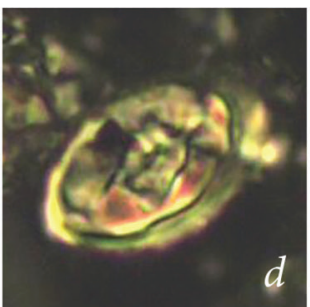

Z. embergeri

Kl-136 (XPL)

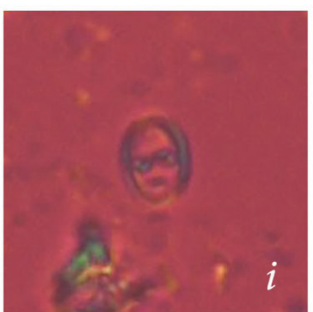

(GP)

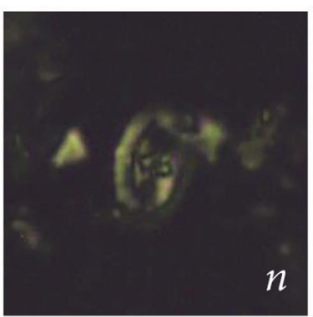

Zeugrhabdotus praesigmoides

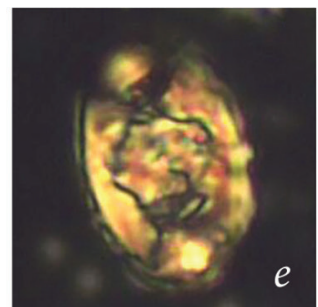

Z. embergeri

Kl-175 (XPL)

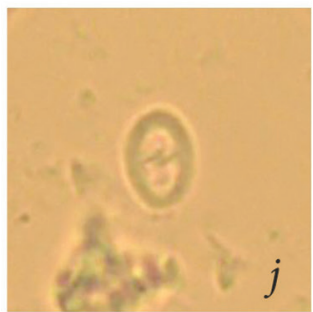

(PPL)

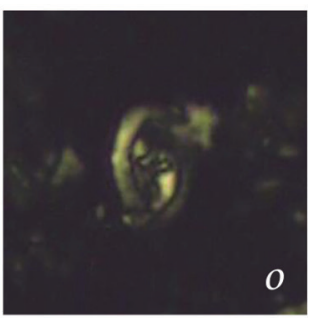

(XPL)
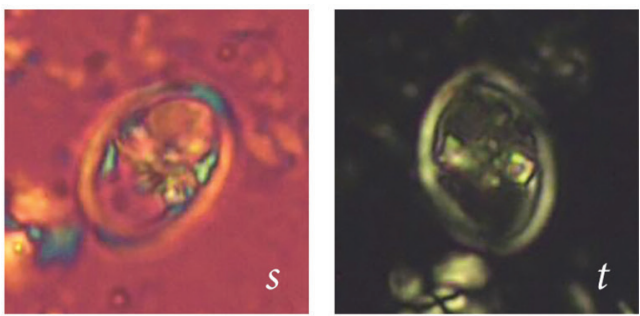

(XPL)

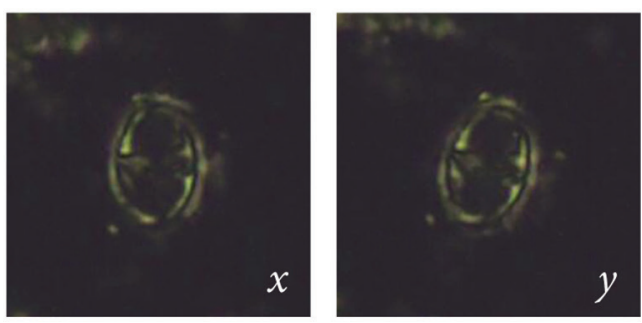

Zeugrhabdotus sigmoides

Kl-183 (XPL)

(XPL)

\section{$5 \mu \mathrm{m}$}

Fig. 6. Calcareous nannofossils from section Kladorub (Chiastozygaceae). Scale bar applies to all images. Abbreviations: XPL cross-polarized light; PPL - plane-polarized light; GP - gypsum plate. 


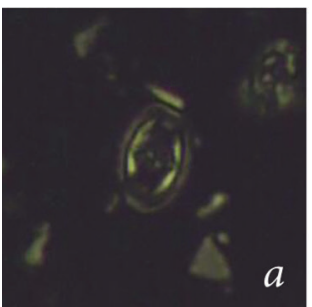

Zeugrhabdotus trivectis Kl-175 (XPL)

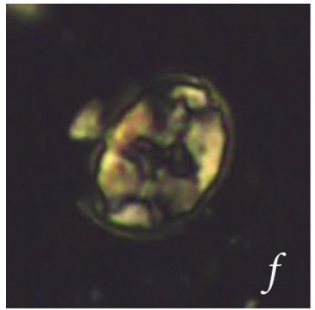

Eiffellithus eximius Kl-77.5 (XPL)

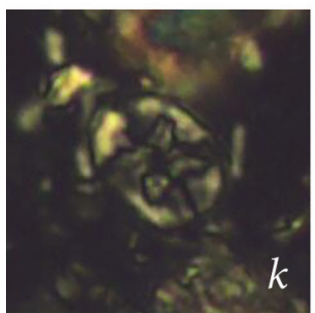

Eiffellithus parallelus K1-93.5 (XPL)

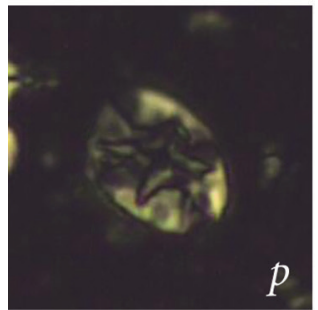

(GP)

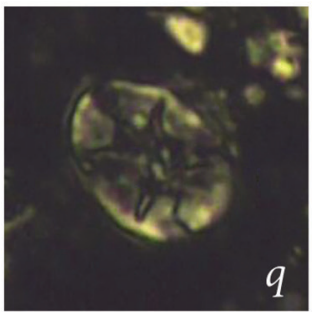

Eiffellithus turriseiffelii Eiffellithus turriseiffeli K1-71 (XPL)

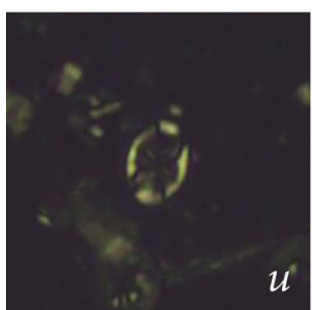

Helicolithus trabeculatus

Kl-77 (XPL)
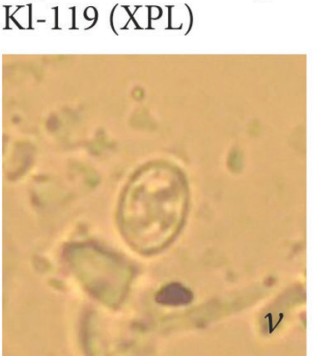

(PPL)

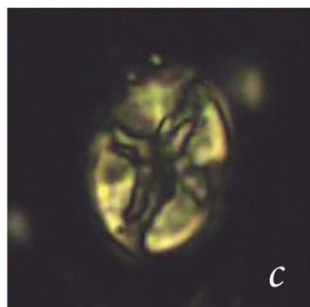

Eiffellithus eximius Kl-72 (XPL)

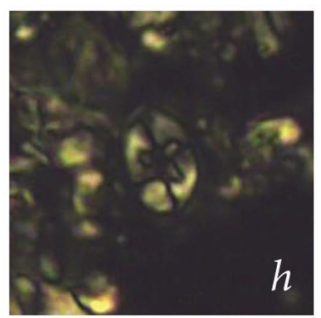

Eiffellithus gorkae Kl-93.5 (XPL)

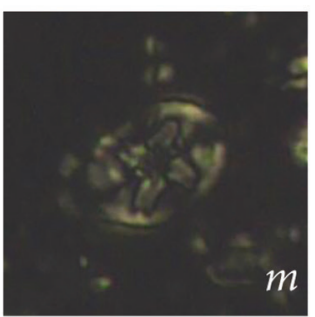

Eiffellithus parallelus K1-155.5 (XPL)

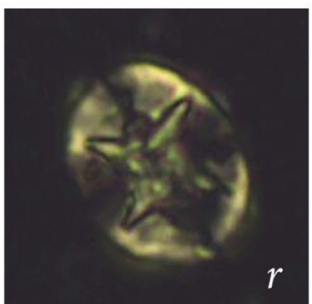

Kl-175 (XPL)

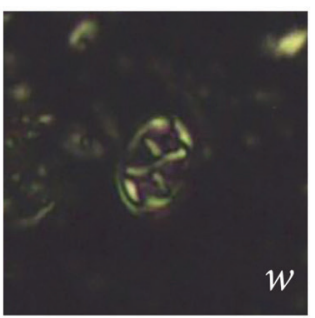

Helico. trabeculatus Kl-168.5 (XPL)
(GP)
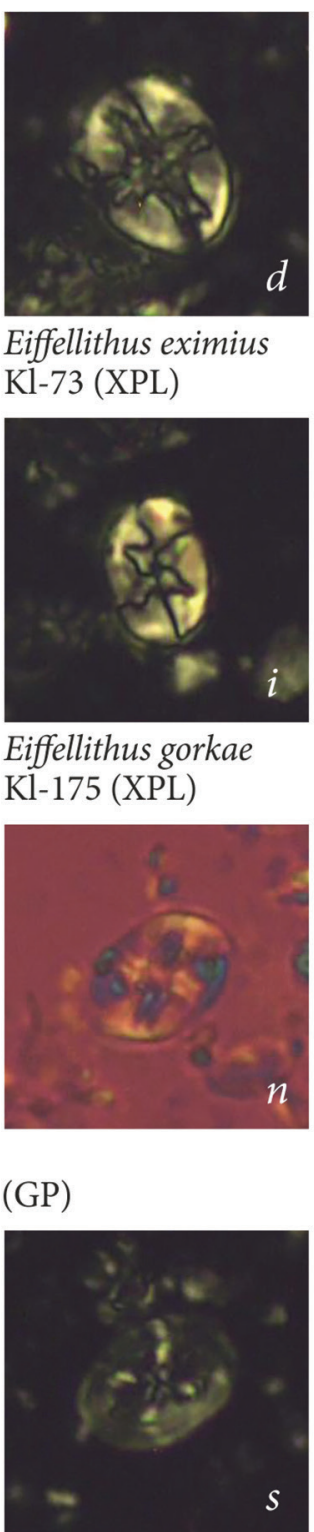

Eiffellithus eximius Kl-73 (XPL)

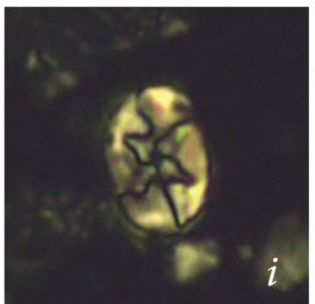

Eiffellithus gorkae Kl-175 (XPL)

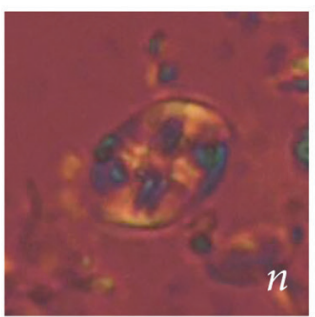

$n$

Eiffellithus parallelus Kl-168.5 (XPL)

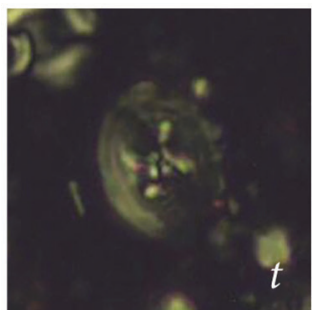

Helicolithus anceps

K1-72 (XPL) K1-71 (XPL)
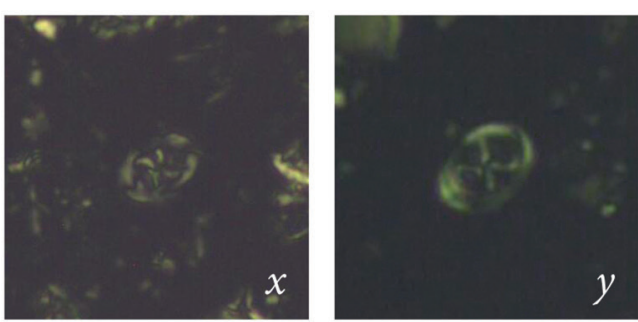

Tegumentum stradneri Teg. stradneri Kl-73 (XPL) Kl-107.5 (XPL)

\section{$5 \mu \mathrm{m}$}

Fig. 7. Calcareous nannofossils from section Kladorub (Chiastozygaceae, Eiffellithaceae). Scale bar applies to all images. Abbreviations: XPL - cross-polarized light; PPL - plane-polarized light; GP - gypsum plate. 

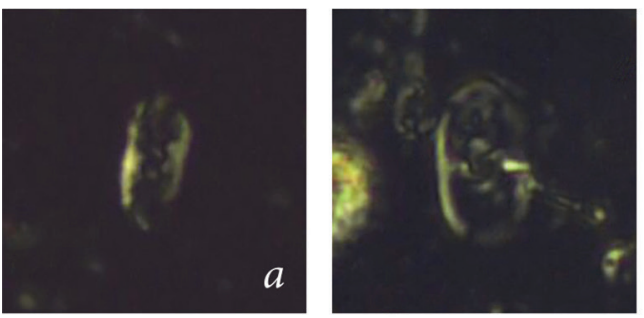

Rhagodiscus angustus Rhagodiscus angustus Kl-71 (XPL)

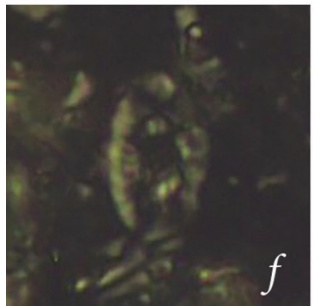
Kl-93.5 (XPL)

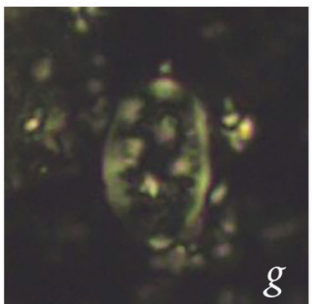

Rhagodiscus splendens Rhagodiscus splendens Kl-77.5 (XPL)

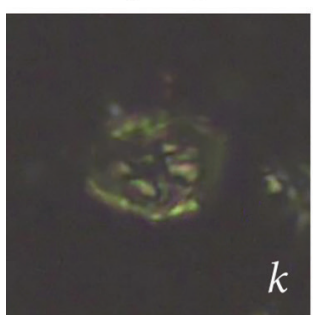

Corollithion completum K1-76 (XPL)

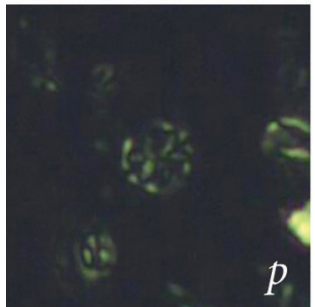

Corollithion exiguum

K1-175 (XPL)

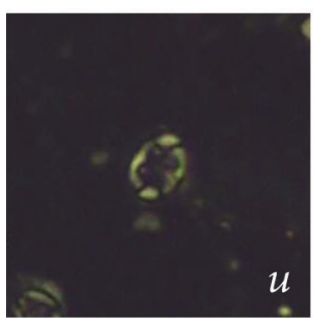

(GP) Kl-168.5 (XPL)

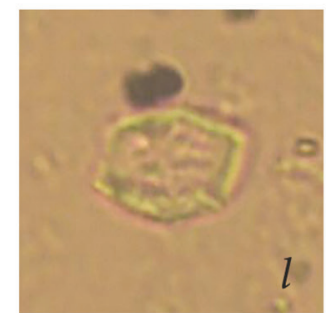

(PPL)
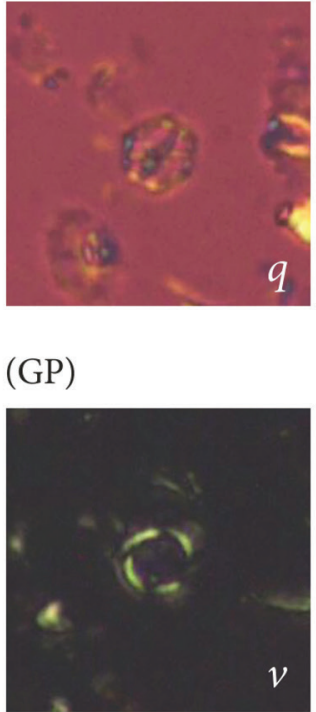

Cor?? madagaskarensis Cylindralithus biarcus K1-72 (XPL)
K1-76 (XPL)

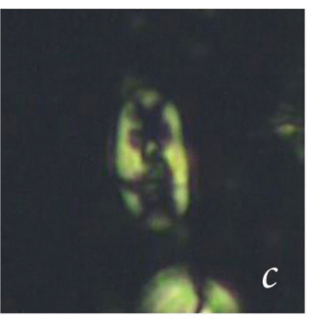

Rhagodiscus angustus K1-173 (XPL)

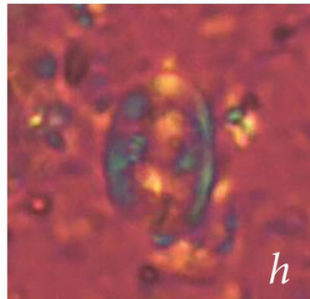

(GP)

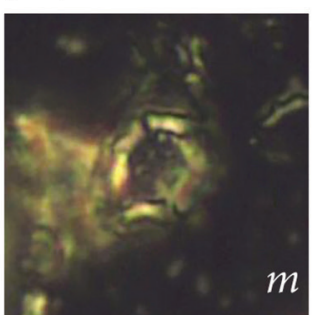

Cor. completum K1-131.5 (XPL)

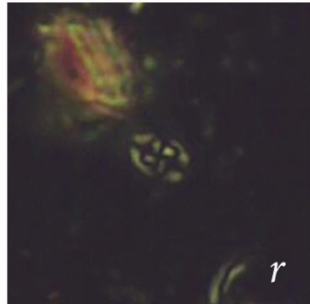

Corollithion? madagaskarensis

$\mathrm{Kl}-72$ (XPL)

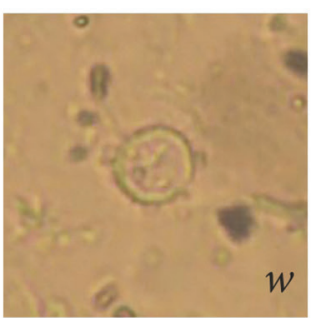

(PPL)

(GP)
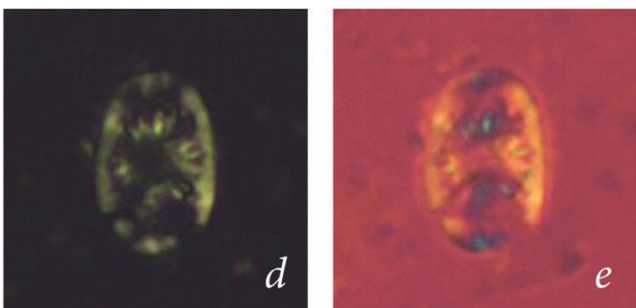

Rhagodiscus splendens Kl-175 (XPL)

(GP)

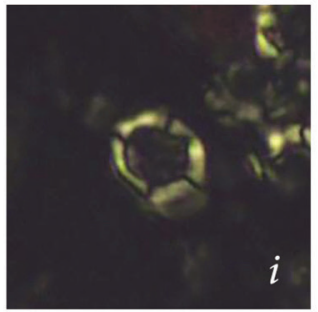

Corollithion completum K1-93.5 (XPL)

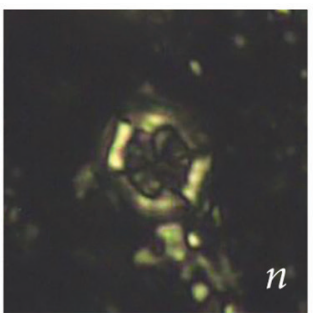

Corollithion completum Kl-151.5 (XPL)

(XPL)
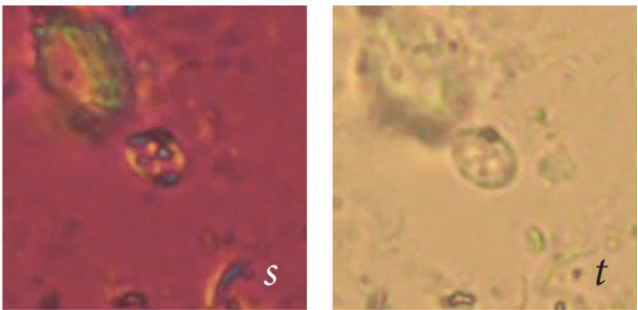

(PPL)
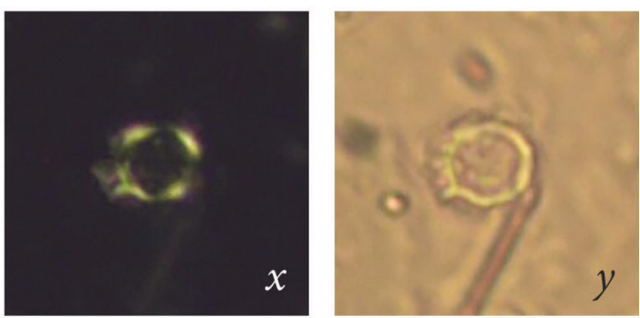

Cylindralithus biarcus

K1-77.5 (XPL)

(PPL)

\section{$5 \mu \mathrm{m}$}

Fig. 8. Calcareous nannofossils from section Kladorub (Eiffellithaceae, Stephanolithiaceae). Scale bar applies to all images. Abbreviations: XPL - cross-polarized light; PPL - plane-polarized light; GP - gypsum plate. 


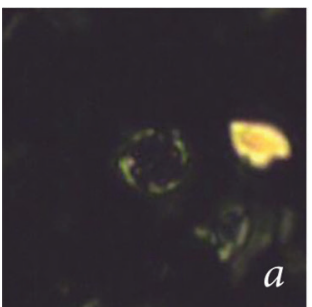

Rotelapillus crenulatus Kl-72 (XPL)

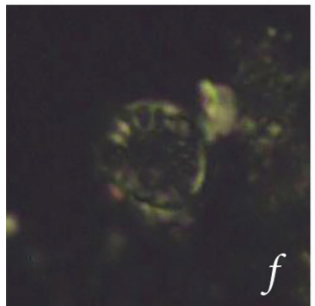

Cribrosphaerella circula K1-96.5 (XPL)

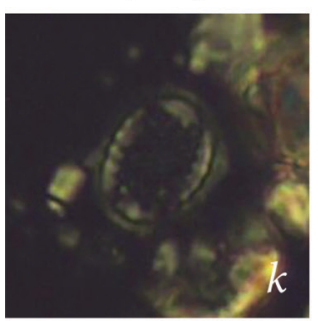

Cribro. ehrenbergii Kl-93.5 (XPL)

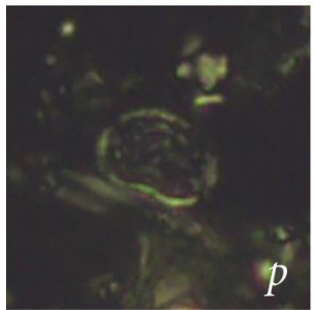

Nephrolithus frequens Kl-168.5 (XPL)

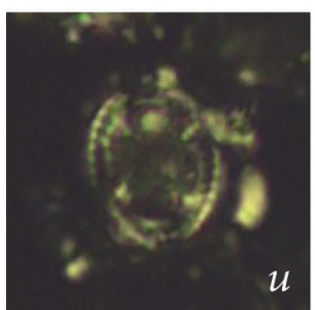

Tetrapodorhabdus decorus Kl-170 (XPL)

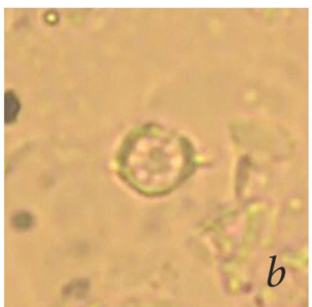

(PPL)

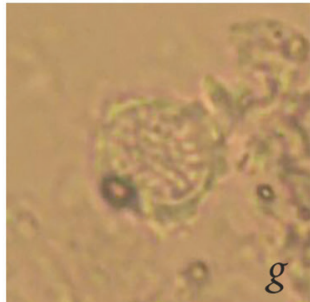

(PPL)

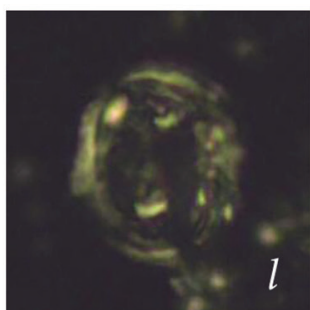

Cribrosphaerella daniae Kl-175 (XPL)

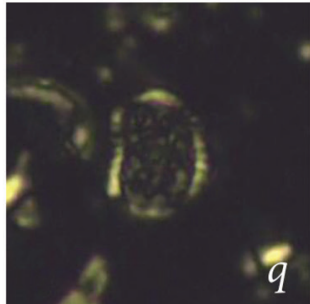

Nephrolithus frequens Kl-170 (XPL)

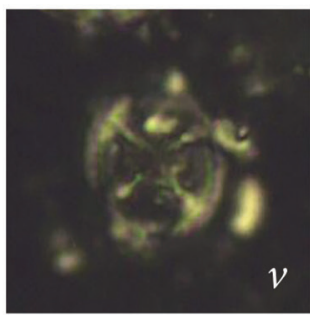

(XPL)

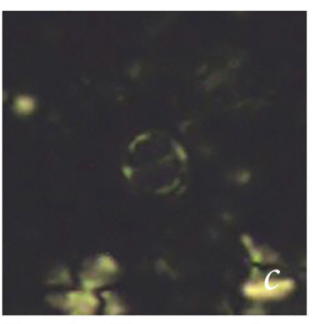

Rotelapillus crenulatus Kl-73 (XPL)

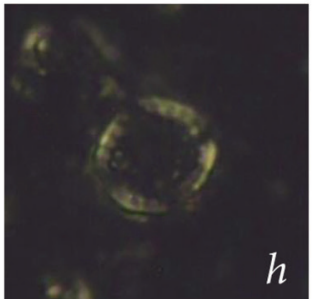

Cribrosphaerella circula K1-175 (XPL)

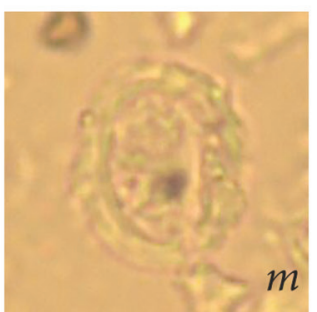

(PPL)

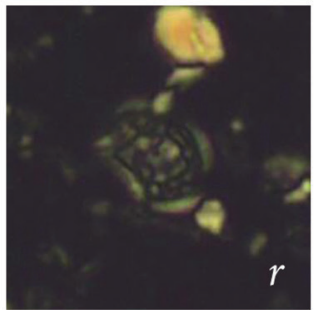

Nephrolithus frequens Kl-170.5 (XPL)

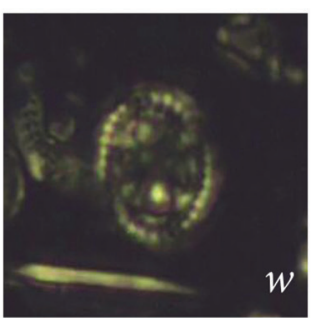

Tetrapodorhabdus decorus Kl-175 (XPL)

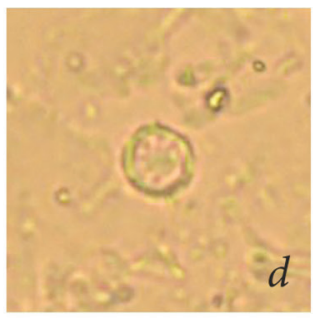

(PPL)

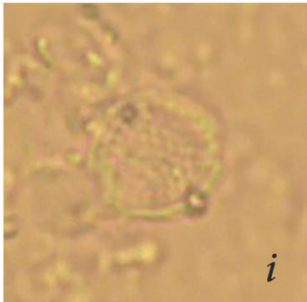

(PPL)

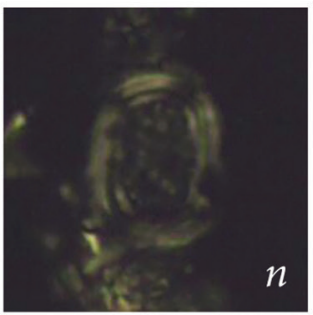

Cribrosphaerella daniae Kl-175 (XPL)

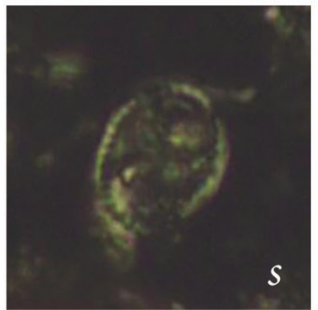

Tetrapodorhabdus decorus Kl-155.5 (XPL)

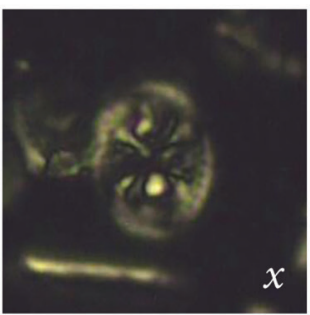

(XPL)

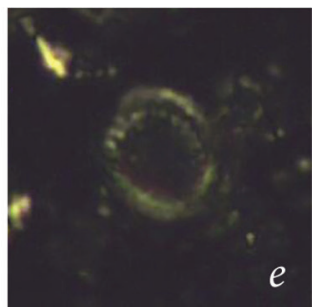

Cribro. circula Kl-72 (XPL)

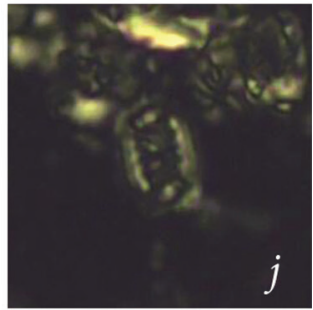

Cribro. ehrenbergii Kl-73 (XPL)

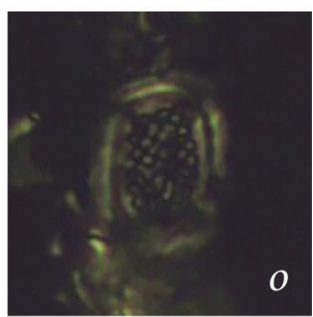

(XPL)

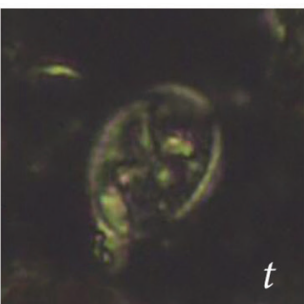

(XPL)

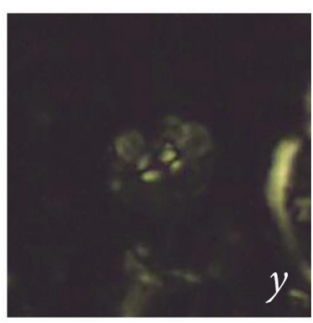

Biscutum constans Kl-71 (XPL)

\section{$5 \mu \mathrm{m}$}

Fig. 9. Calcareous nannofossils from section Kladorub (Stephanolithiaceae, Axopodorhabdaceae, Biscutaceae). Scale bar applies to all images. Abbreviations: XPL - cross-polarized light; PPL - plane-polarized light. 


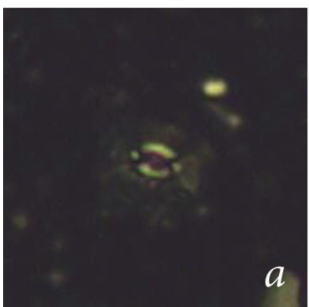

Biscutum constans Kl-175 (XPL)

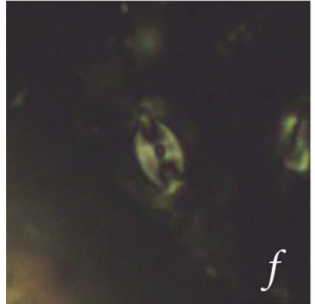

Biscutum notaculum Kl-131 (XPL)

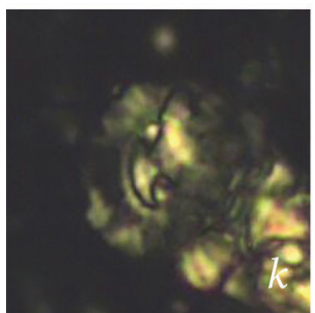

Biscutum notaculum Kl-73 (XPL)

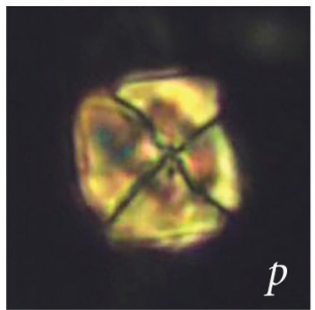

Petrarhabdus copulatus Kl-72 (XPL)

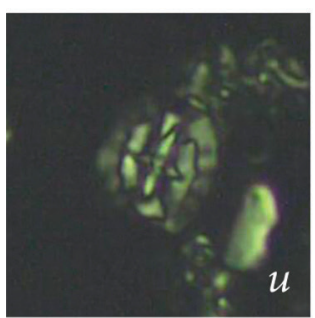

Prediscosphaera arkhangelskyi Kl-93.5 (XPL)

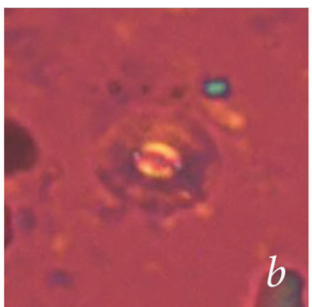

(GP)

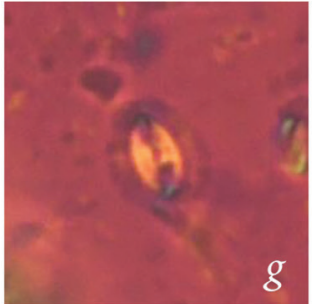

(GP)

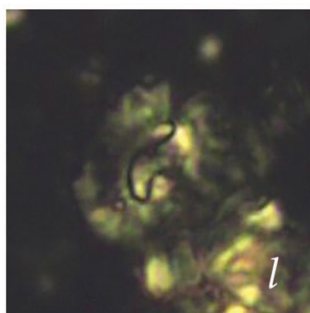

(XPL)

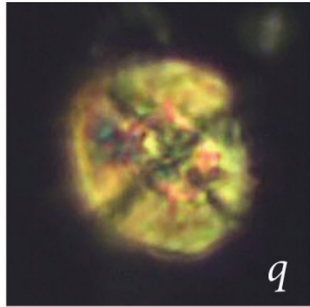

(XPL)

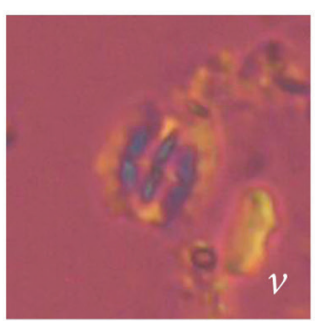

(GP)

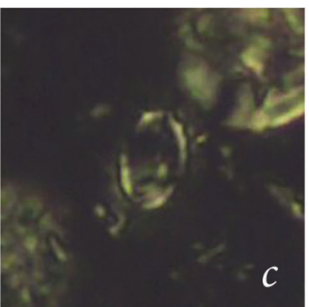

Biscutum magnum Kl-131 (XPL)

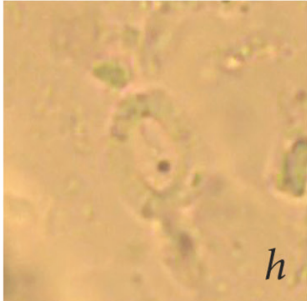

(PPL)

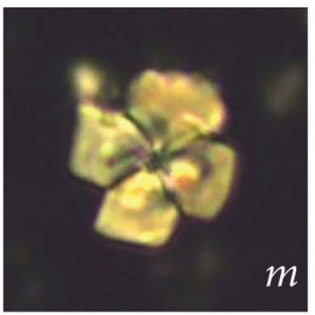

Petrarhabdus copulatus K1-77 (XPL)

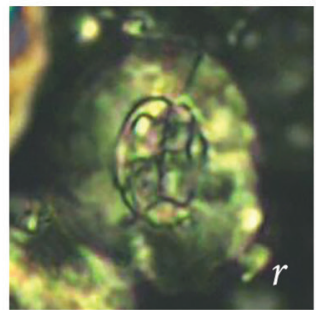

Petrarhabdus? vietus $\mathrm{Kl}-77$ (XPL)

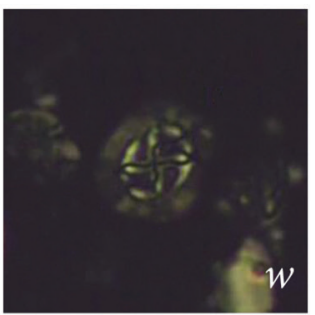

Predisco. cretacea Kl-71 (XPL)

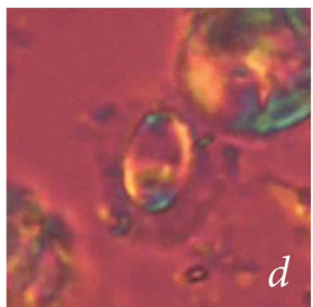

(GP)

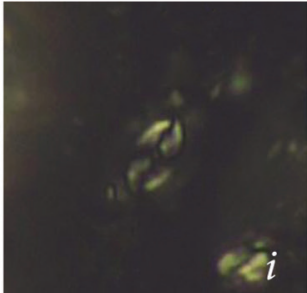

(XPL)

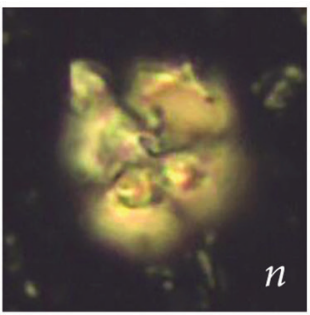

(XPL)

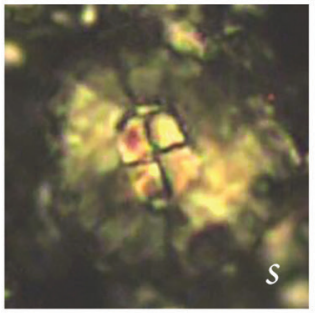

Petrarhabdus? vietus Kl-121 (XPL)

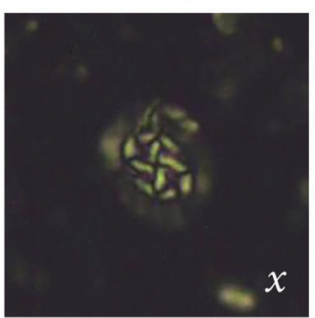

Predisco. cretacea Kl-175 (XPL)

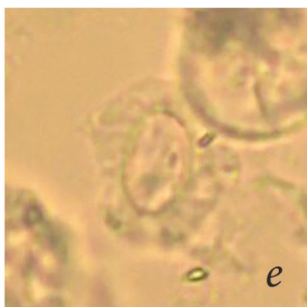

(PPL)

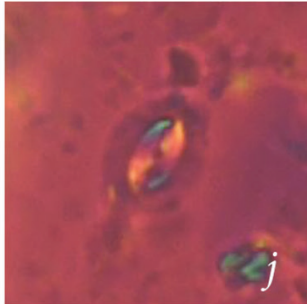

(GP)

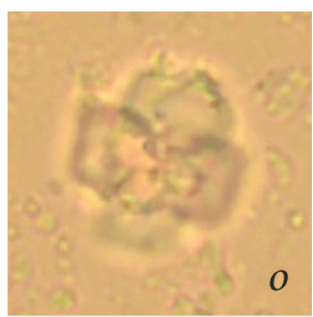

(PPL)

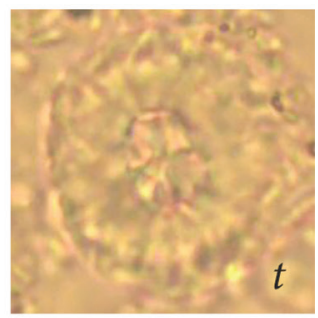

(PPL)

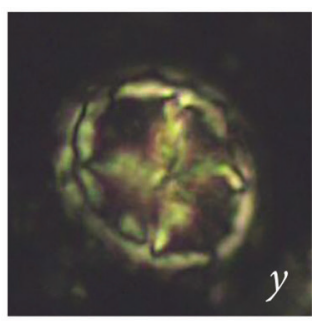

Predisco. grandis Kl-168.5 (XPL)

\section{$5 \mu \mathrm{m}$}

Fig. 10. Calcareous nannofossils from section Kladorub (Biscutaceae, Prediscosphaeraceae). Scale bar applies to all images. Abbreviations: XPL - cross-polarized light; PPL - plane-polarized light; GP - gypsum plate. 


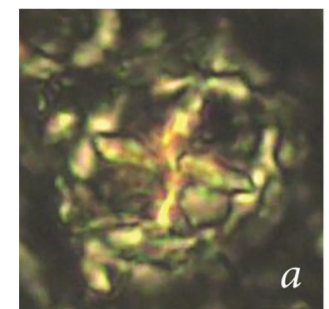

Predisco. grandis Kl-175 (XPL)

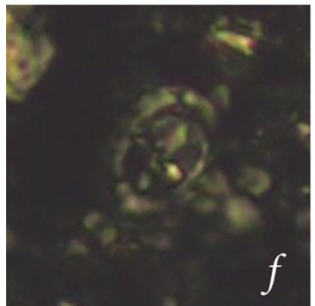

Prediscosphaera mgayae

Kl-155.5 (XPL)

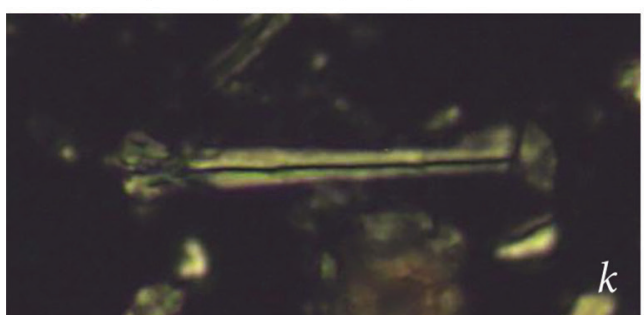

Prediscosphaera microrhabdulina spine Kl-93.5 (XPL)

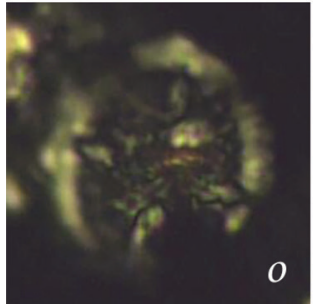

Cretarhabdus conicus Kl-93.5 (XPL)

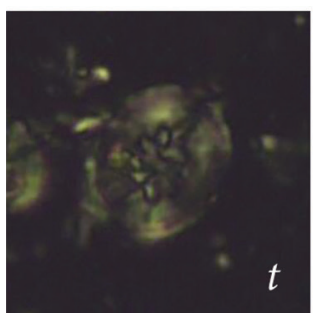

Retecapsa ficula Kl-72 (XPL)

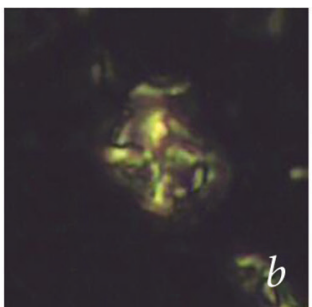
Kl-123 (XPL)

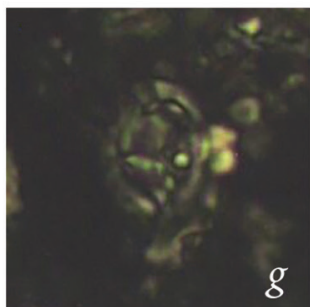

$g$

(XPL)

$k$

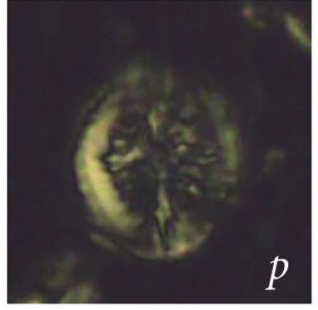

Cretarhabdus conicus Kl-123 (XPL)

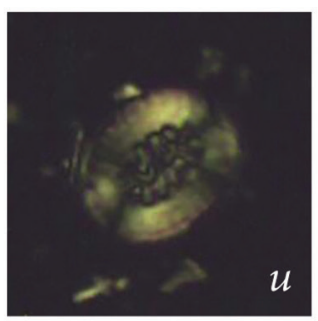

Retecapsa ficula Kl-175 (XPL)

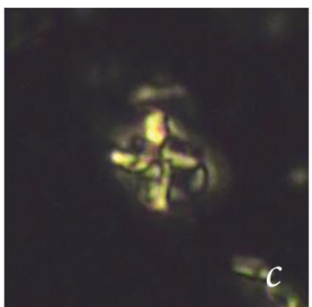

Prediscosphaera majungae

\section{Pred}

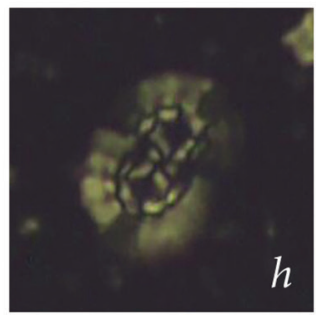

Prediscosphaera microrhabdulina

Kl-175 (XPL)

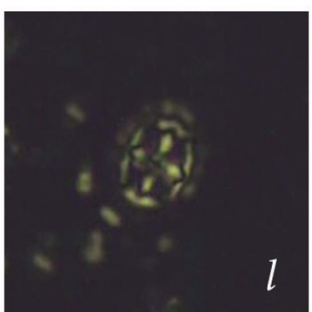

Predisco. spinosa

Kl-170.5 (XPL)

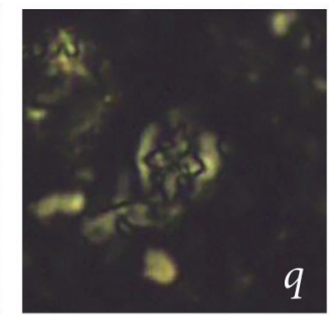

Ret. angustiforata Kl-72 (XPL)

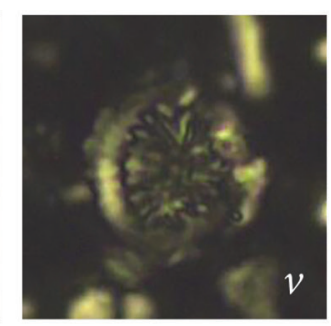

Retecapsa surirella Kl-132 (XPL)

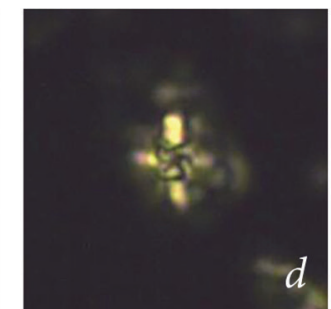

(XPL)

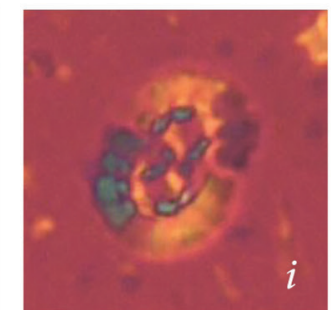

(GP)

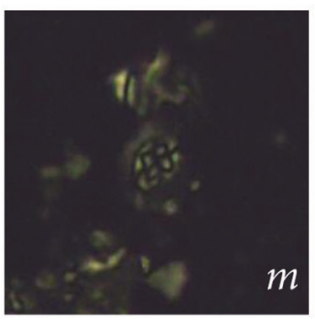

Prediscosphaera stoveri Kl-93.5 (XPL)

(XPL)

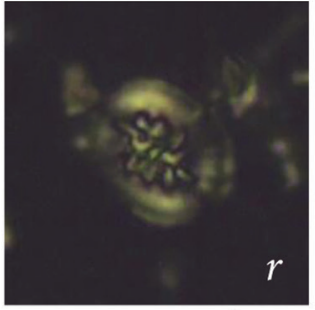

Retecapsa crenulata Kl-72 (XPL)

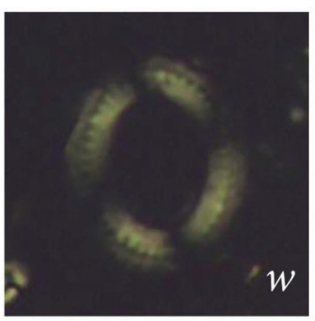
Kl-175 (XPL)

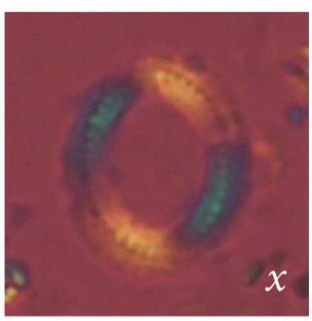

Manivitella pemmatoidea Kl-96.5 (XPL)

Predisco. majungae Kl-175 (XPL)

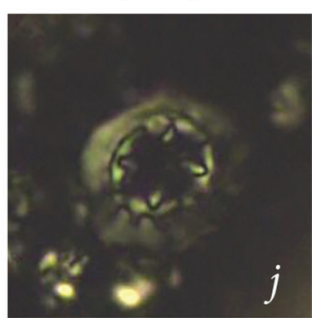

Predisco. ponticula Kl-122.5 (XPL)
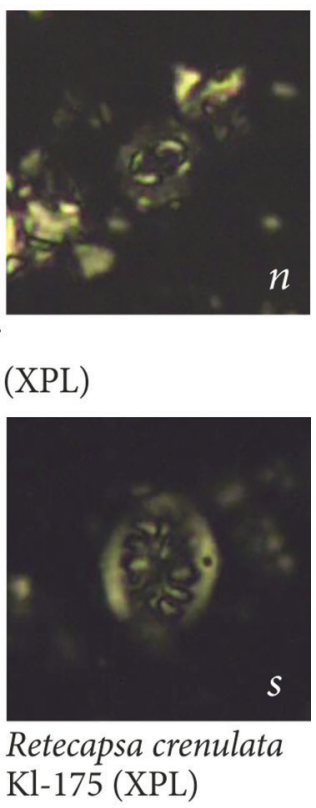

$s$

\section{$5 \mu \mathrm{m}$}

Fig. 11. Calcareous nannofossils from section Kladorub (Prediscosphaeraceae, Cretarhabdaceae). Scale bar applies to all images. Abbreviations: XPL - cross-polarized light; GP - gypsum plate. 


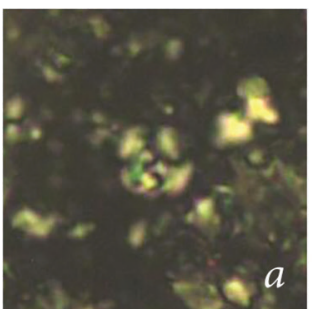

Cyclagelosphaera alta K1-182 (XPL)

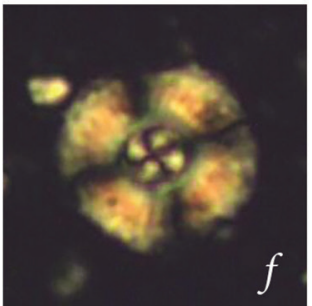

Cyclagelosphaera margerelii Kl-77.5 (XPL)

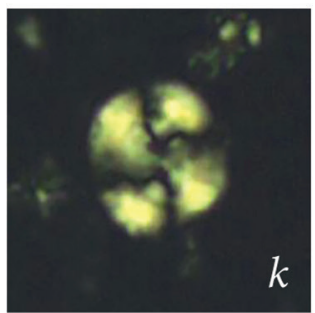

Watznaueria biporta Kl-175 (XPL)

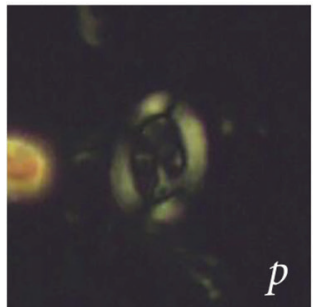

Arkhangelskiella confusa Kl-96.5 (XPL)

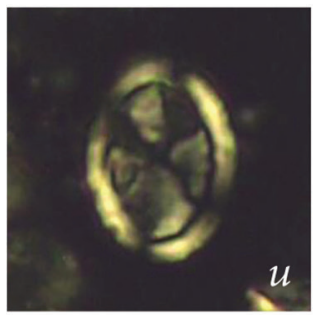

Arkh. cymbiformis Kl-152.5 (XPL)

(GP)

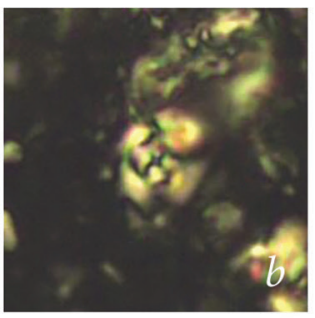

Cyclagelosphaera alta K1-183 (XPL)
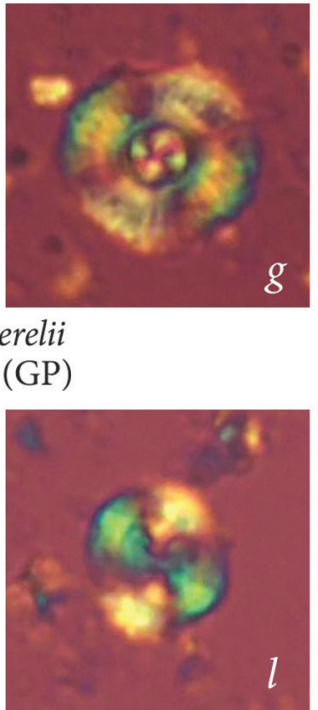

(GP)

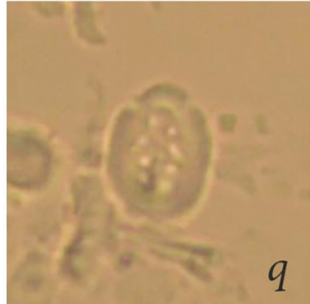

(PPL)

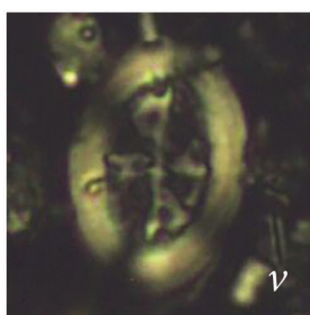

Arkhangelkskiella maastrichtiensis Kl-170 (XPL)

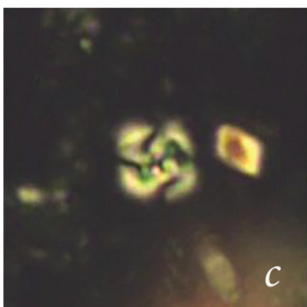

Cyclagelosphaera alta Kl-183 (XPL)

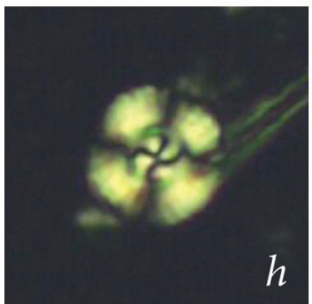

Cyclagelosphaera reinhardtii Kl-175 (XPL)

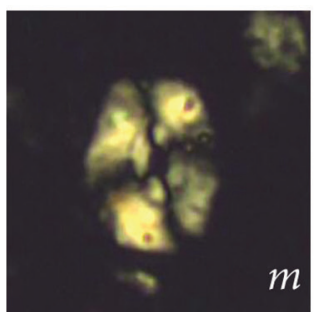

W. fossacincta Kl-123 (XPL)

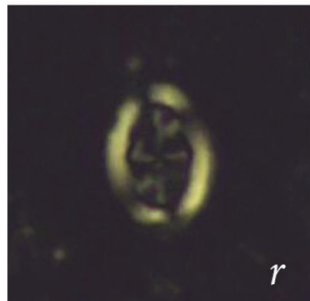

Arkh. confusa Kl-123 (XPL)

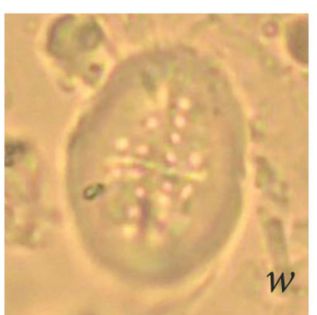

(PPL)

(GP)

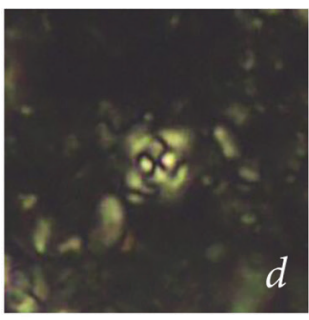

Cyclagelosphaera alta Kl-183 (XPL)
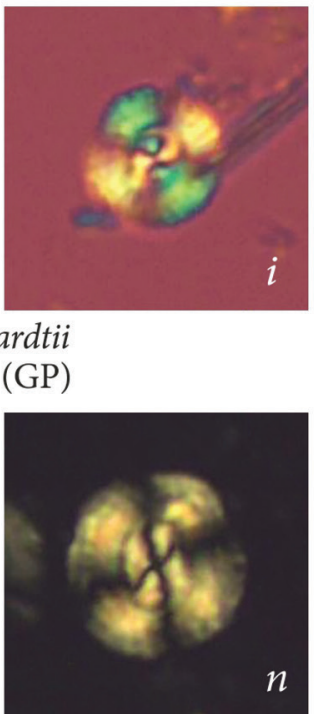

W. manivitiae

Kl-77.5 (XPL)

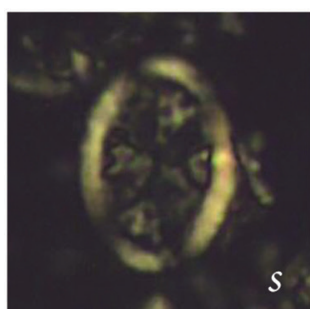

Arkhangelskiella cymbiformis K1-77.5 (XPL)

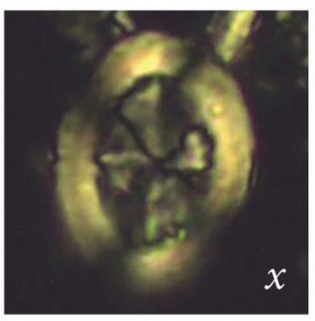

Arkhangelskiella maastrichtiensis Kl-170.5 (XPL)

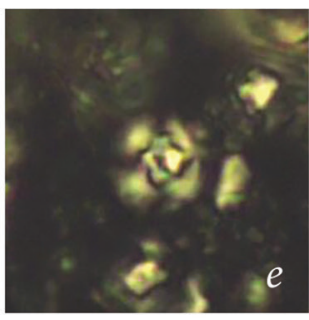

Cyclagelosphaera alta Kl-183 (XPL)

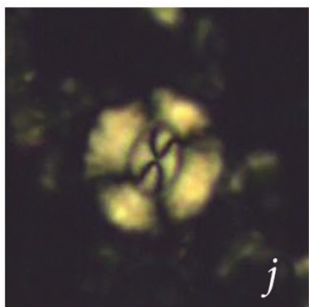

W. barnesiae Kl-77 (XPL)

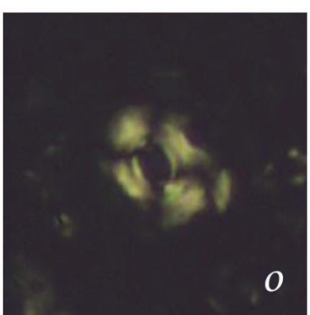

Watznaueria ovata Kl-76 (XPL)

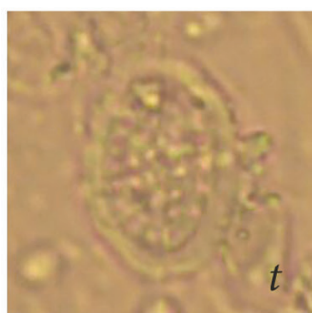

(PPL)

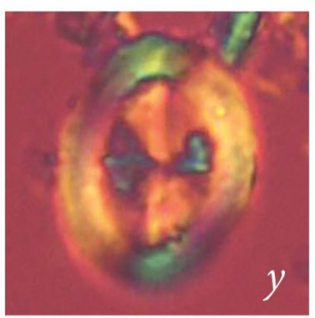

(GP)

$5 \mu \mathrm{m}$

Fig. 12. Calcareous nannofossils from section Kladorub (Watznaueriaceae, Arkhangelskiellaceae). Scale bar applies to all images. Abbreviations: XPL - cross-polarized light; PPL - plane-polarized light; GP - gypsum plate. 


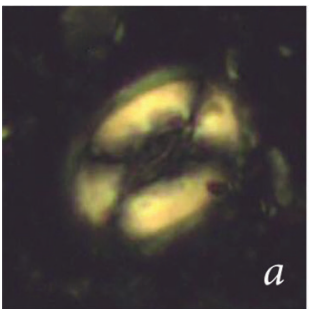

Br. parca constricta Kl-72 (XPL)

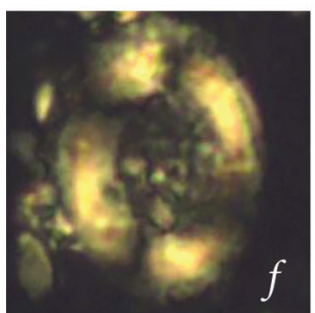

Br. parca parca $\mathrm{Kl}-77$ (XPL)

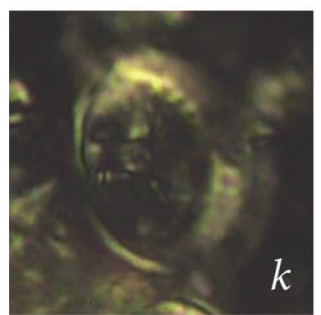

Kamptnerius magnificus $\mathrm{Kl}-170$ (XPL)

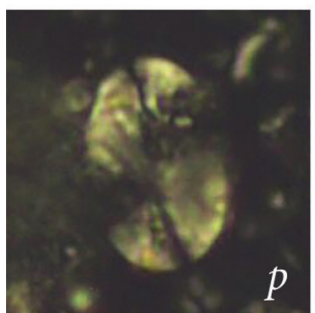

Prolatipatella multicarinata Kl-168.5 (XPL)

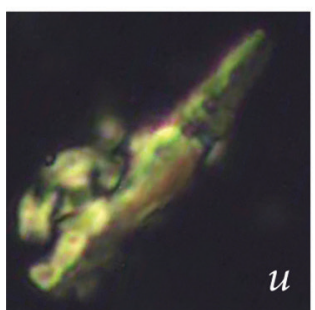

Acuturris scotus Kl-70 (XPL)

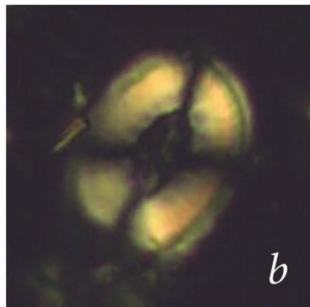

Broinsonia parca constricta Kl-76 (XPL)

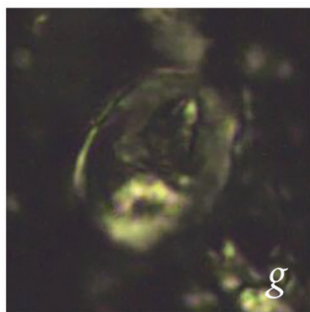

G. segmentatum Kl-77.5 (XPL)

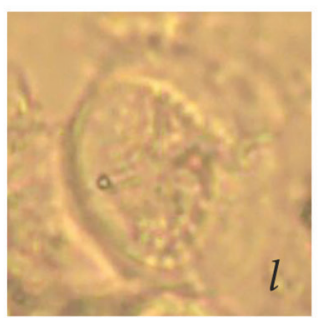

(PPL)

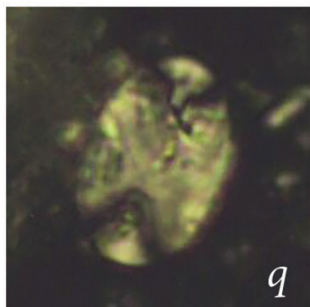

(XPL)

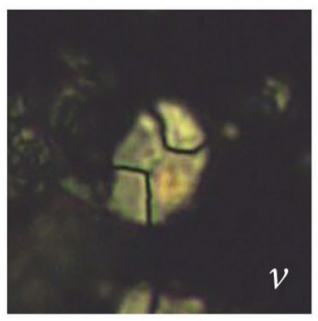

Calculites obscurus Kl-119 (XPL)

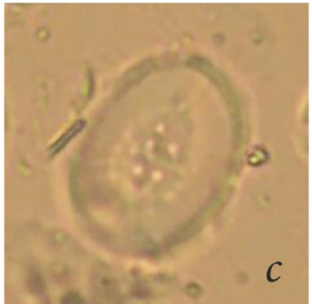

(PPL)

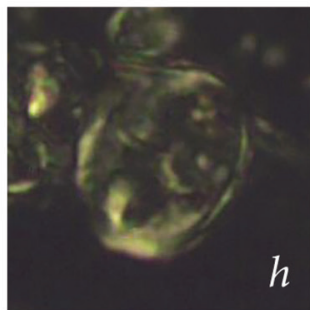

G. segmentatum Kl-170 (XPL)

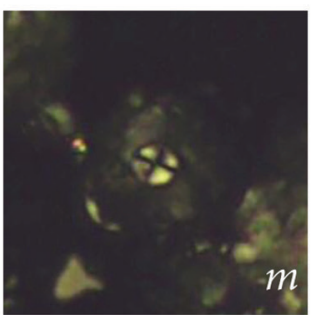

Markalius inversus Kl-183 (XPL)

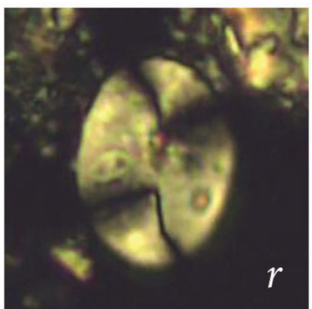

Prolatipatella multicarinata Kl-175.5 (XPL)

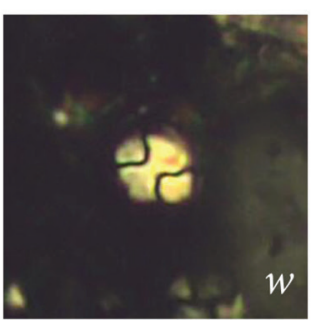

Calculites obscurus Kl-152.5 (XPL)

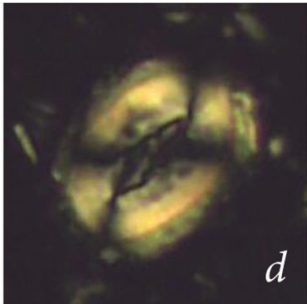

Br. parca constricta K1-77.5 (XPL)

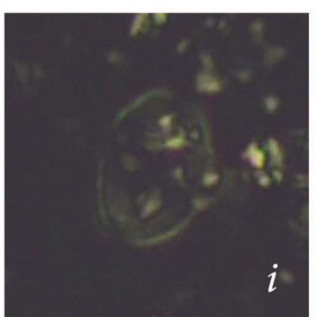

G. segmentatum Kl-170.5 (XPL)

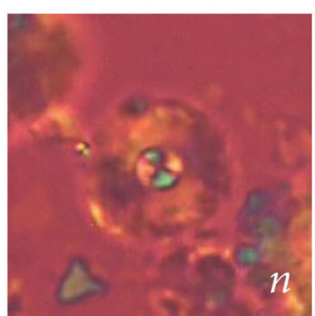

(GP)

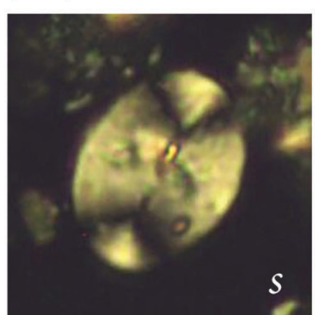

(XPL)

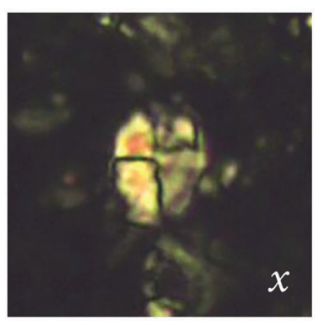

Calculites ovalis Kl-131.5 (XPL)

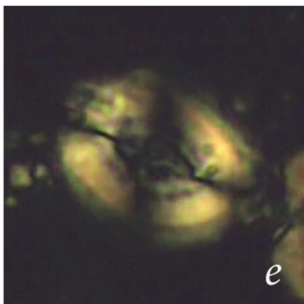

Br. parca constricta Kl-96.5 (XPL)

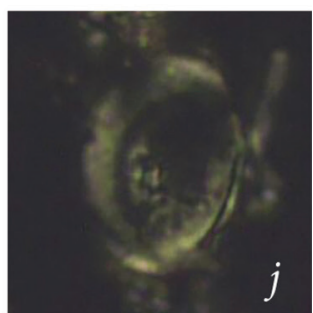

K. magnificus Kl-73 (XPL)

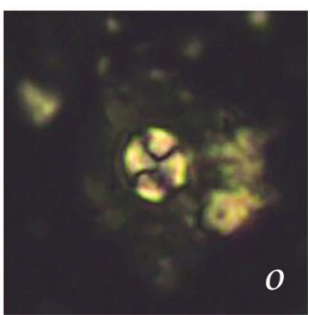

Markalius inversus Kl-183 (XPL)

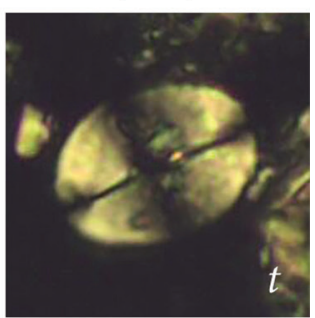

(XPL)

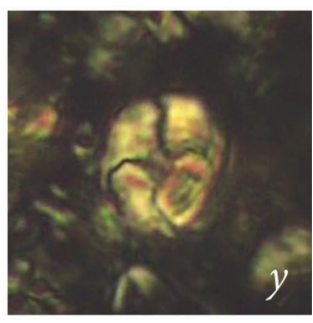

Calculites ovalis Kl-155.5 (XPL)

$5 \mu \mathrm{m}$

Fig. 13. Calcareous nannofossils from section Kladorub (Arkhangelskiellaceae, Kamptneriaceae, heterococcoliths incertae sedis, holococcoliths). Scale bar applies to all images. Abbreviations: XPL - cross-polarized light; PPL - plane-polarized light; GP gypsum plate. 


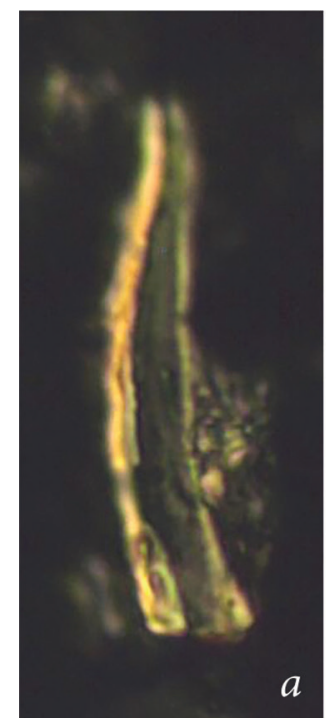

Lucianorh. cayeuxii Kl-93.5 (XPL)

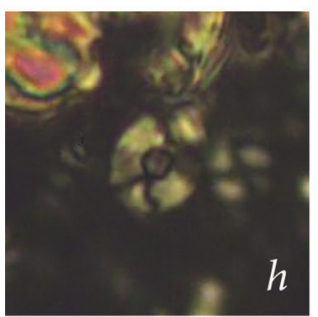

Russelia bukryi

Kl-155.5 (XPL)

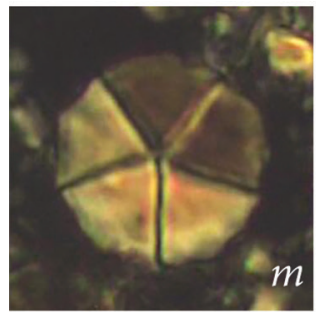

Micrantholithus disculus

K1-155.5 (XPL)

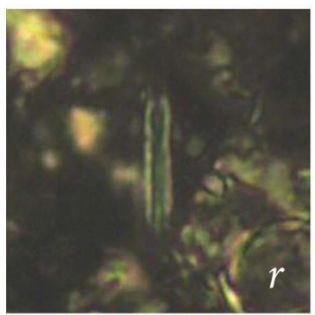

Lithraphidites praequadratus

Kl-154.5 (XPL) (PPL)

(XPL)

(PPL)
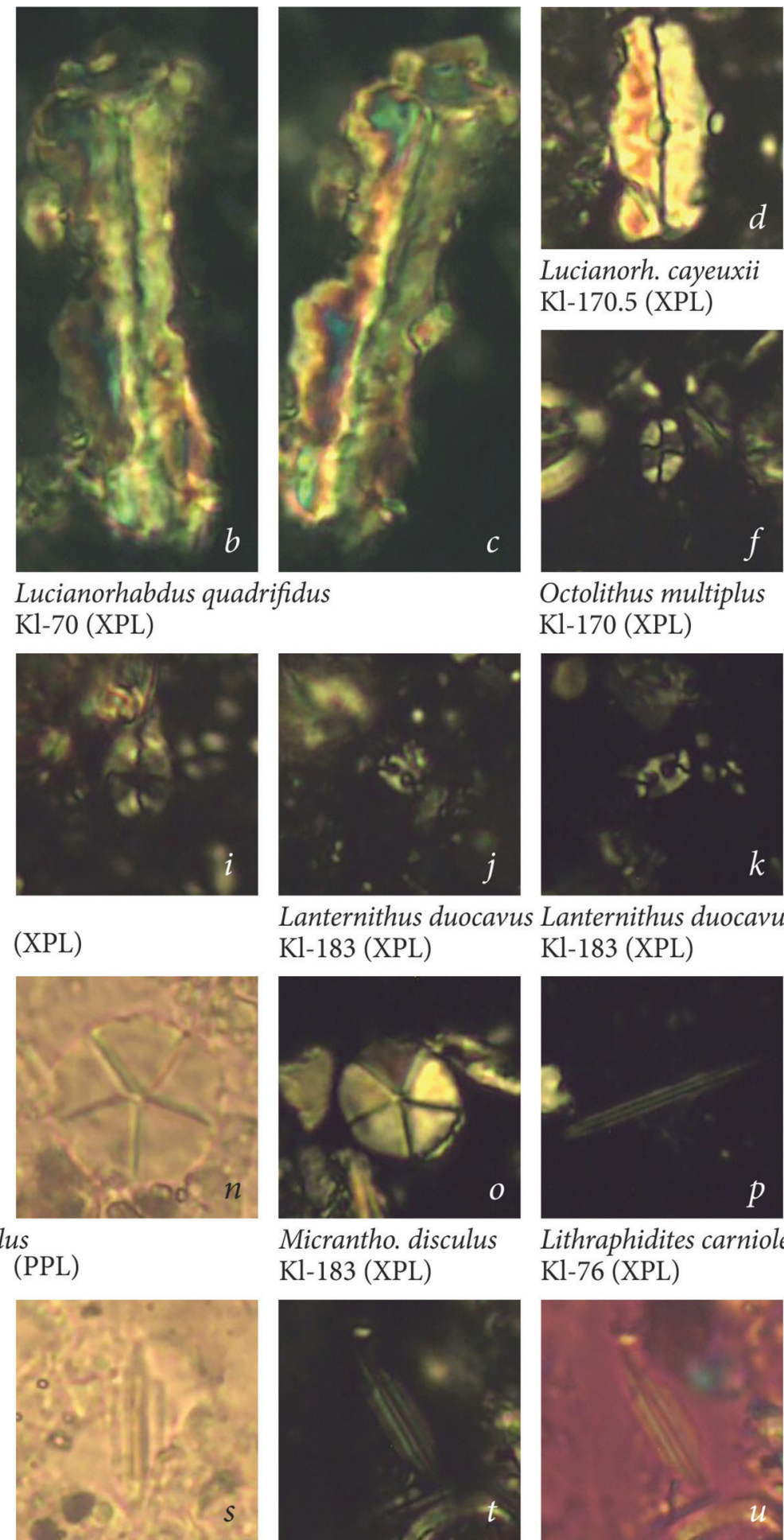
Kl-183 (XPL)
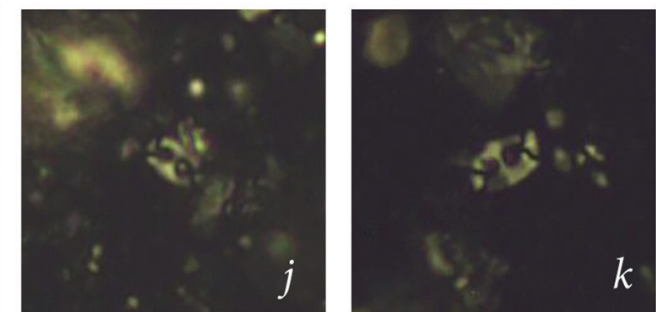

Lanternithus duocavus Lanternithus duocavus Braarudo. bigelowii

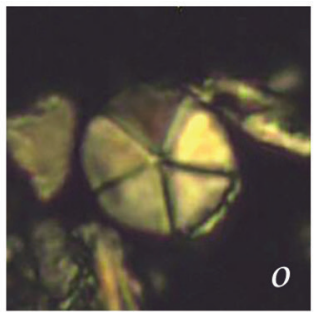

Micrantho. disculus Kl-183 (XPL)

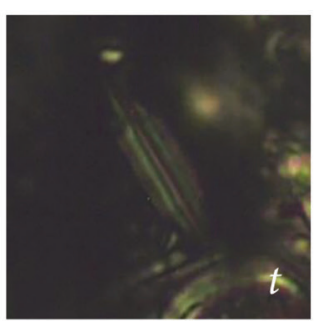

Lithraphidites praequadratus Kl-155.5 (XPL) (GP) Kl-154.5 (XPL)

Lithraphidites carniolensis K1-76 (XPL)

(PPL)

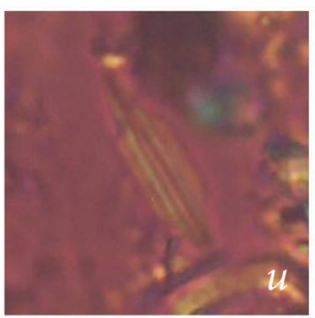

(PPL)
Lucianorh. cayeuxii Kl-170.5 (XPL)

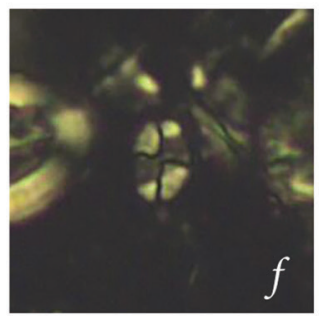

Octolithus multiplus Kl-170 (XPL)

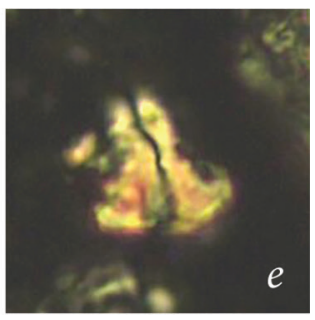

Luc. maleformis Kl-132 (XPL)

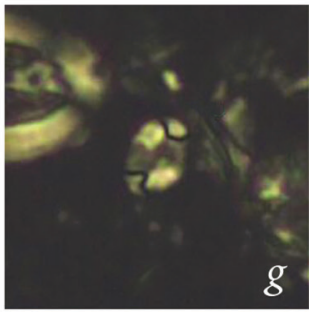

(XPL)
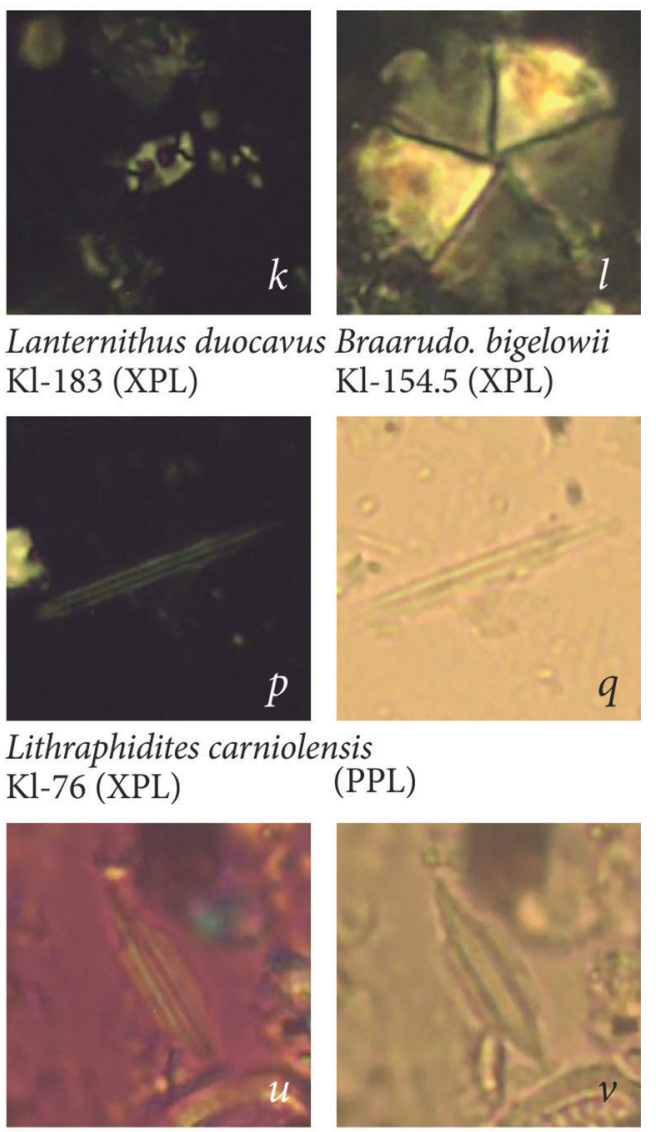

\section{$5 \mu \mathrm{m}$}

Fig. 14. Calcareous nannofossils from section Kladorub (holococcoliths, nannoliths incertae sedis, nannoliths - Braarudosphaeraceae, Microrhabdulaceae). Scale bar applies to all images. Abbreviations: XPL - cross-polarized light; PPL - plane-polarized light; GP - gypsum plate. 


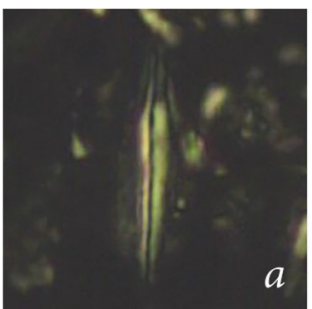

Lithraphidites praequadratus

Kl-159 (XPL)

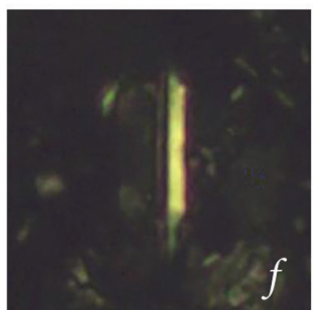

Lithraphidites quadratus

Kl-169 (XPL)

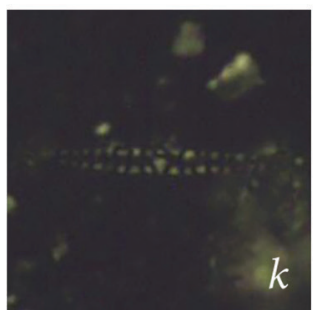

Microrhabdulus belgicus

K1-73 (XPL)

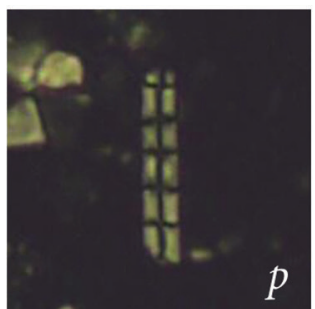

Microrhabdulus decoratus

Kl-175 (XPL)

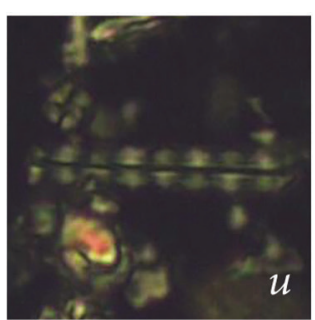

Microrhabdulus undosus

Kl-175 (XPL)

(GP)

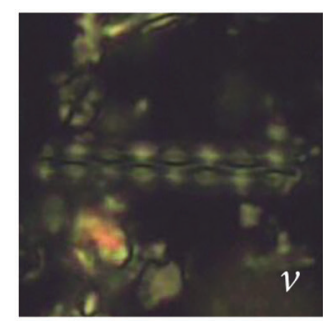

(XPL)

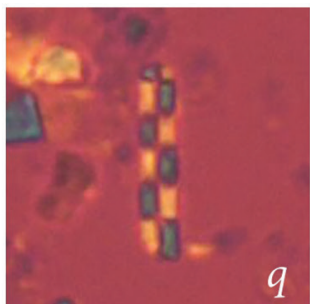

$q$
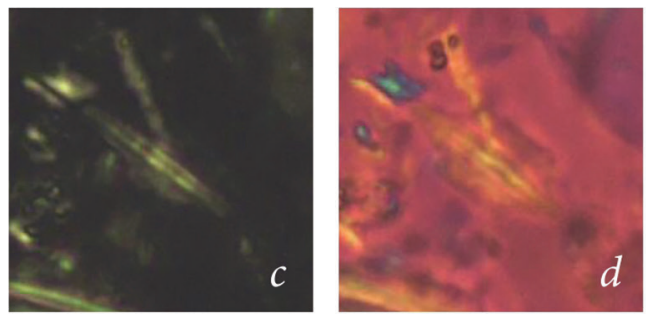

Lithraphidites quadratus

Kl-159 (XPL)

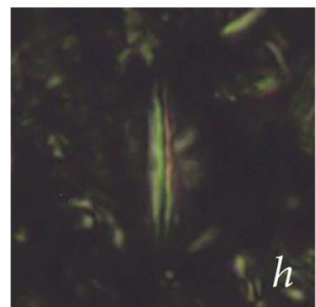

(GP)

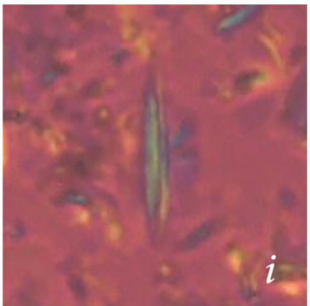

Lithraphidites quadratus Kl-170 (XPL)

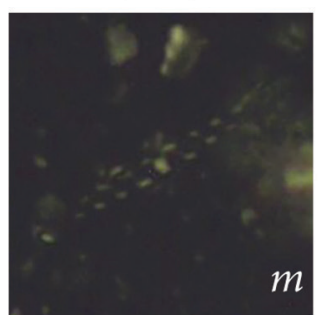

(GP)

(PPL)
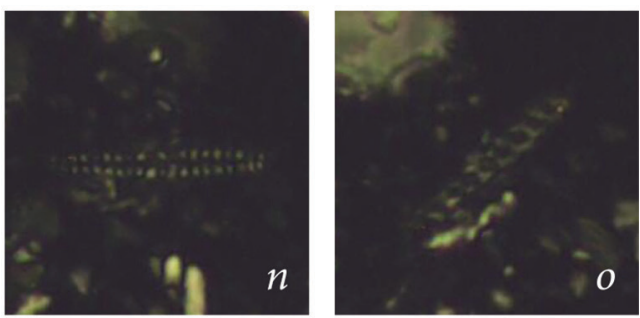

Microrhabdulus belgicus

K1-175 (XPL)

(XPL)
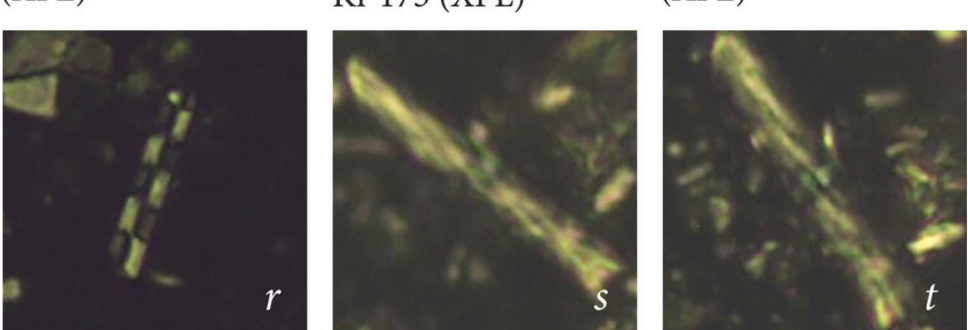

Microrhabdulus helicoideus

(XPL)

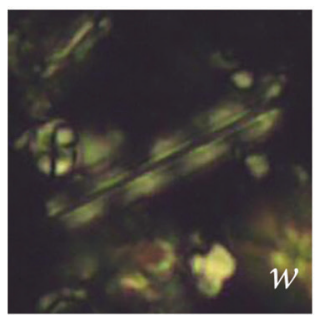

(XPL)
Kl-93.5 (XPL)

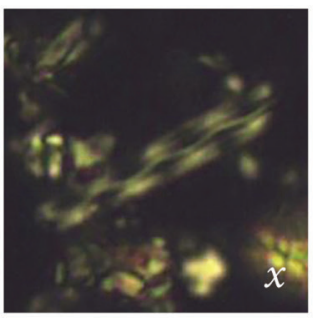

(XPL)
(XPL)

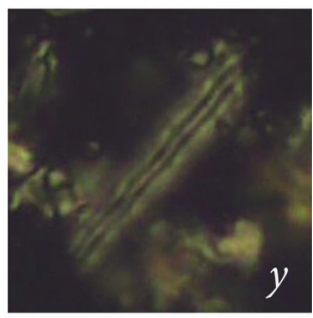

(XPL)

\section{$5 \mu \mathrm{m}$}

Fig. 15. Calcareous nannofossils from section Kladorub (nannoliths - Microrhabdulaceae). Scale bar applies to all images. Abbreviations: XPL - cross-polarized light; PPL - plane-polarized light; GP - gypsum plate. 


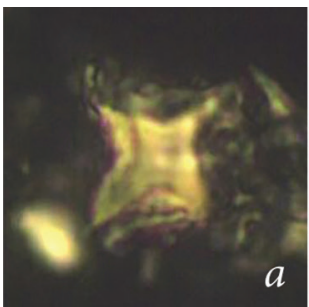

Micula concava Kl-152.5

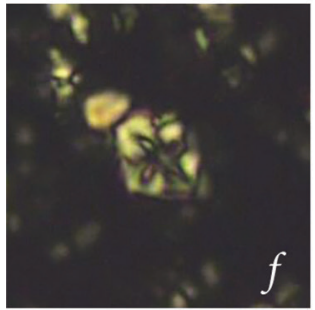

Micula cubiformis

Kl-175

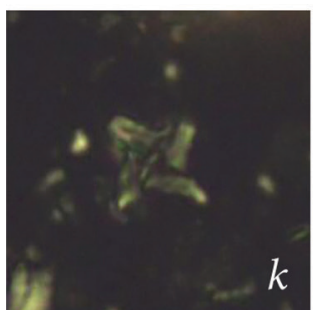

Micula murus

Kl-168.5

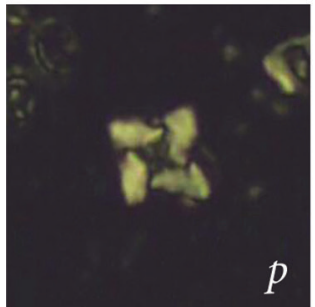

Micula murus

Kl-175

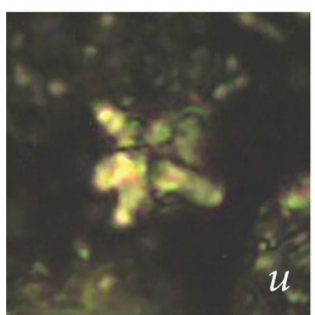

Micula prinsii

Kl-173
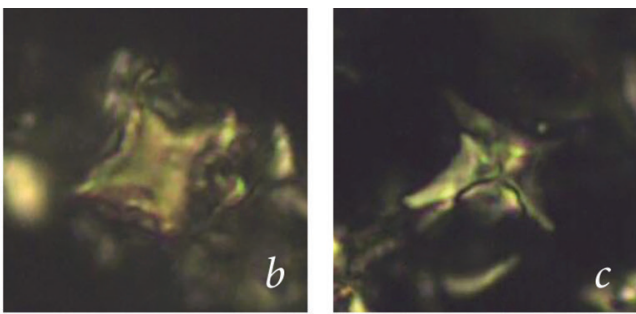

Micula concava

Kl-175

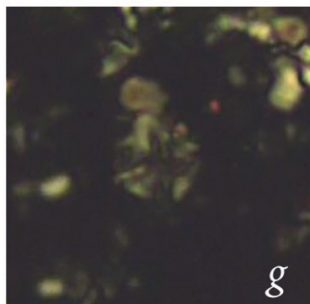

$g$

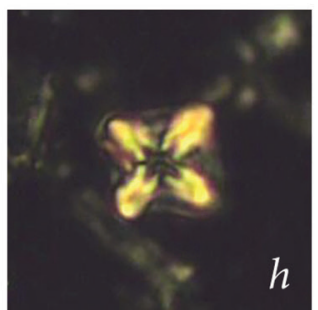

Micula staurophora Kl-72

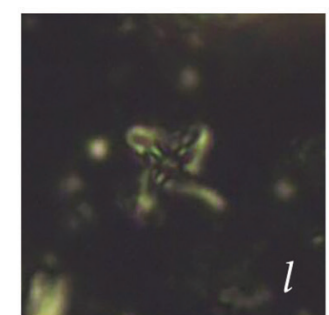

$l$

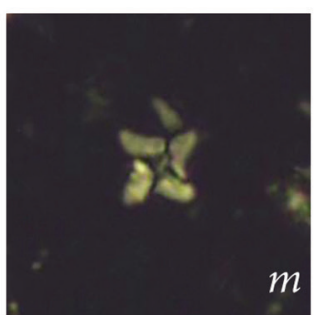

Micula murus Kl-175

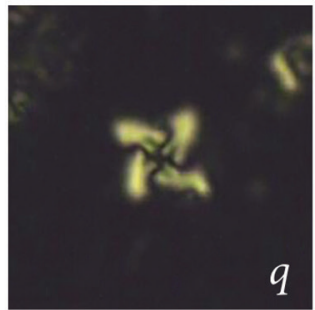

$q$

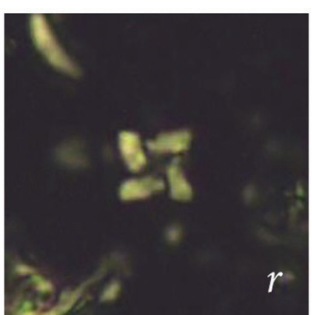

Micula murus

Kl-175

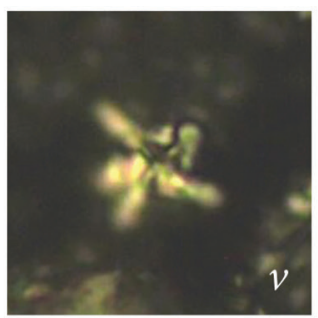

$v$

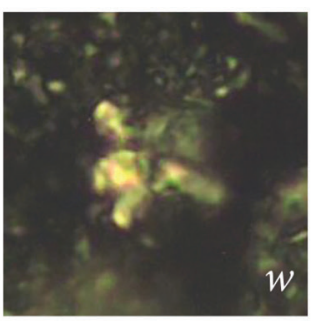

$w$

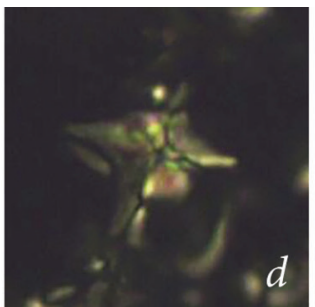

(1)

Micula cubiformis Kl-72

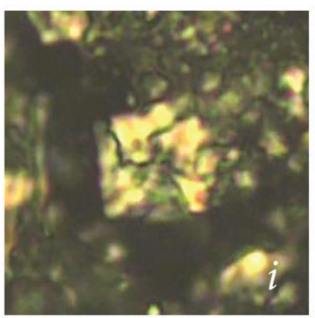

Micula swastica Kl-175.5
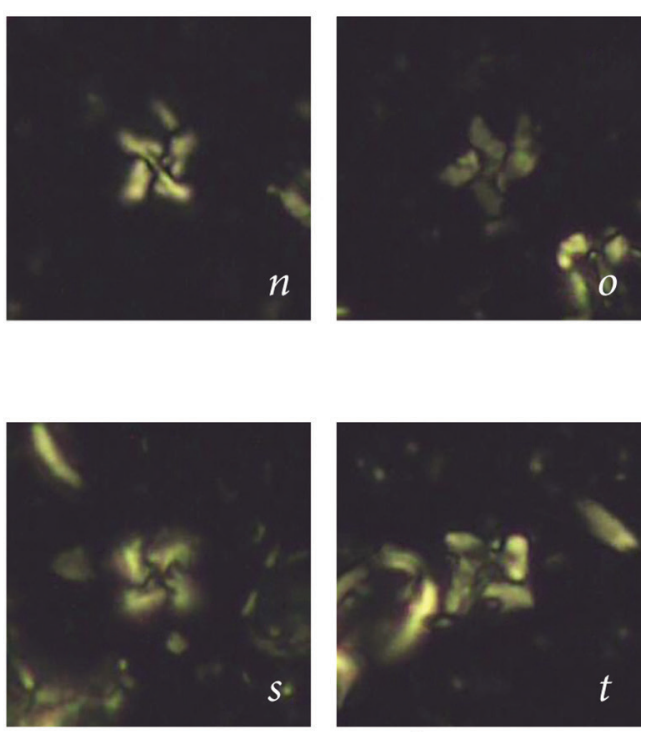

Micula murus Kl-175.5

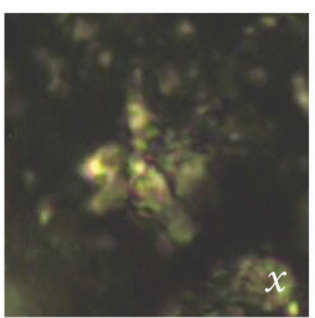

$x$

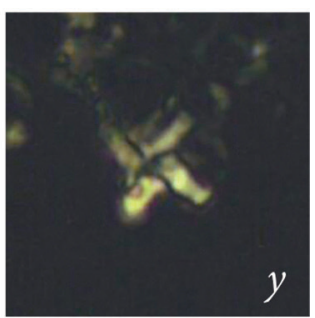

Micula prinsii Kl-173.5

\section{$5 \mu \mathrm{m}$}

Fig. 16. Calcareous nannofossils from section Kladorub (nannoliths - Polycyclolithaceae). All images are uniformly enlarged at $\times 2000$ and taken in cross-polarized light. 


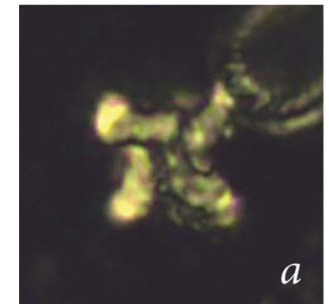

Micula prinsii

Kl-175 (XPL)

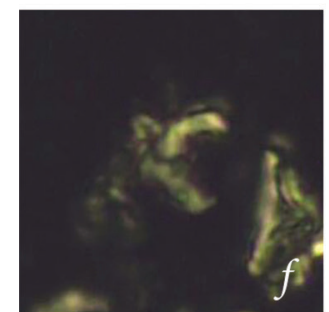

Micula prinsii

Kl-175.5 (XPL)

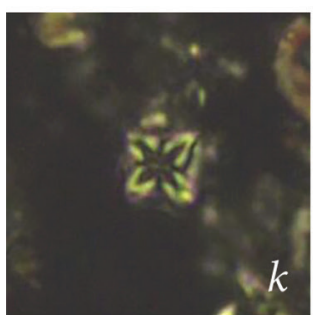

Micula staurophora Kl-121 (XPL)

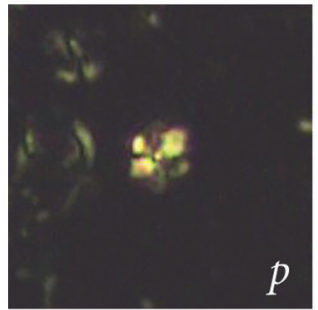

Rucinolithus hayi

Kl-76 (XPL)

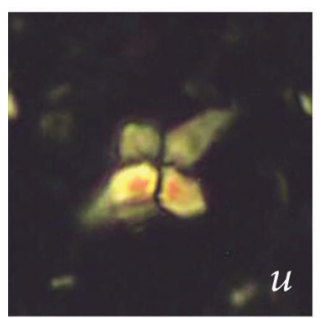

Uniplanarius gothicus Kl-72 (XPL)

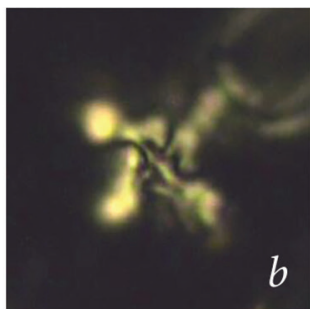

(XPL)

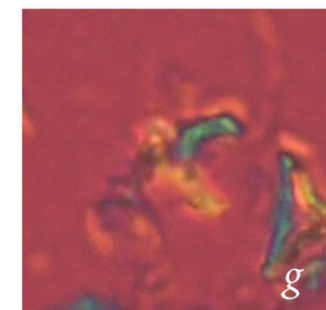

(GP)

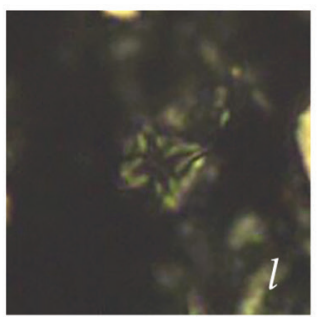

(XPL)

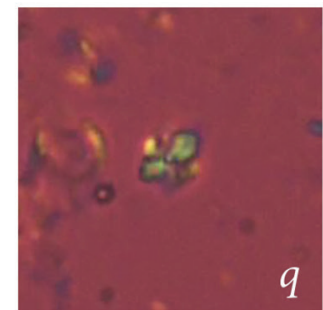

(GP)

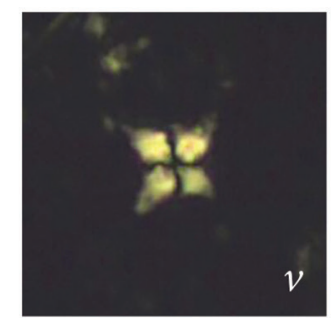

Uniplanarius gothicus Kl-73 (XPL)

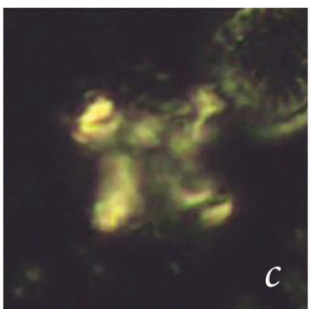

(XPL)

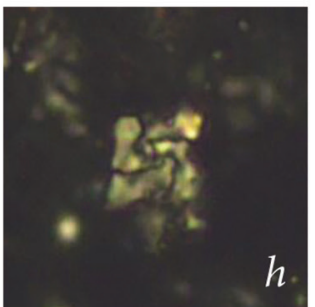

Micula cf. M. swastica Kl-175 (XPL)

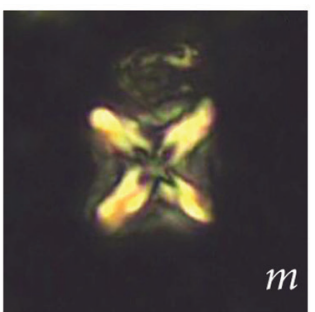

Micula staurophora Kl-175 (XPL)

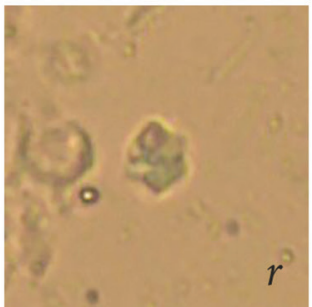

(PPL)

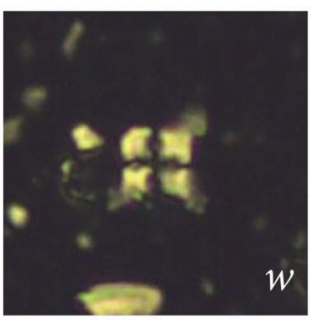

Uniplanarius gothicus Kl-77 (XPL)

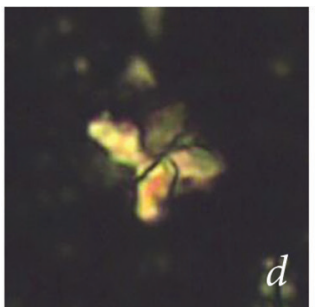

Micula prinsii Kl-175 (XPL)

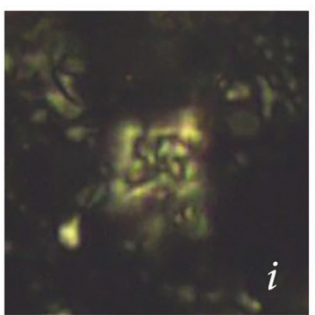

(XPL)

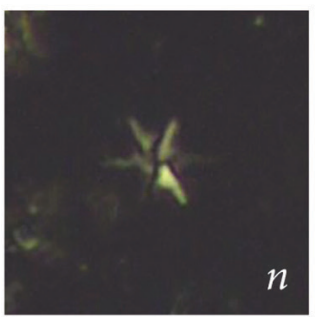

Rucinolithus? magnus K1-76 (XPL)

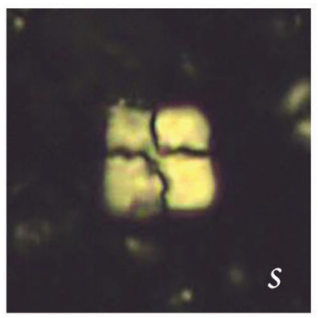

Uniplanarius gothicus Kl-72 (XPL)

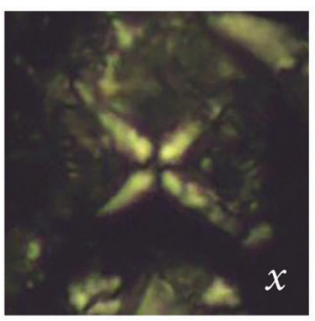

Uniplanarius sissinghii $\mathrm{Kl}-72$ (XPL)
(XPL)

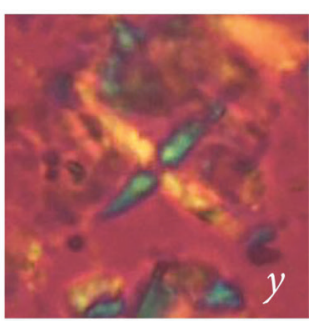

(XPL)

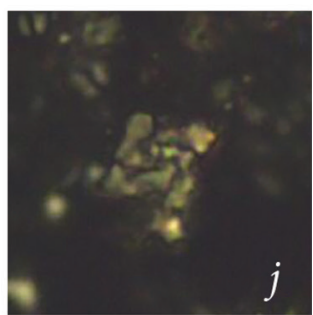

(XPL)

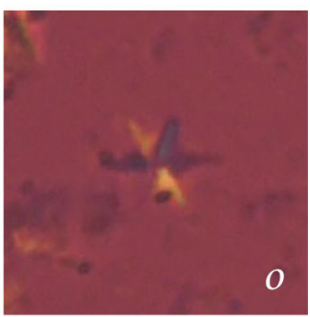

(GP)

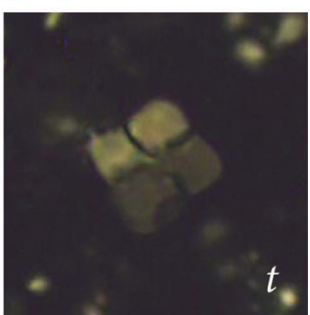

(GP)

\section{$5 \mu \mathrm{m}$}

Fig. 17. Calcareous nannofossils from section Kladorub (nannoliths - Polycyclolithaceae, incertae sedis). Scale bar applies to all images. Abbreviations: XPL - cross-polarized light; PPL - plane-polarized light; GP - gypsum plate. 

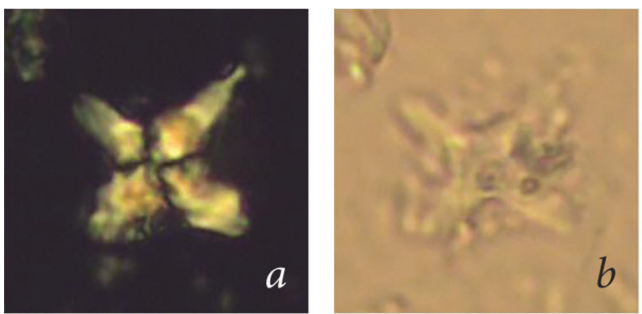

Uniplanarius sissinghii

Kl-77.5 (XPL)

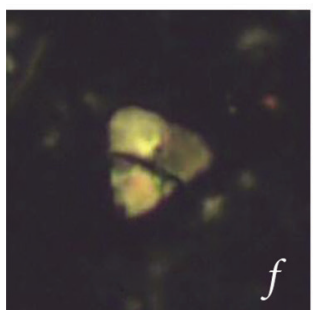

Uniplanarius trifidus $\mathrm{Kl}-72$ (XPL)

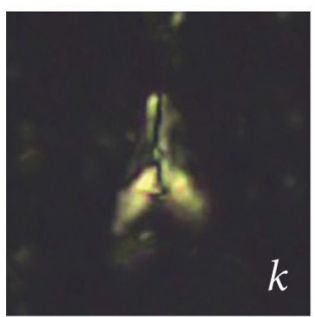

Ceratolithoides aculeus K1-73 (XPL)

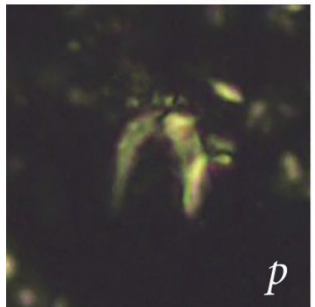

Ceratolithoides kamptneri

Kl-169 (XPL)

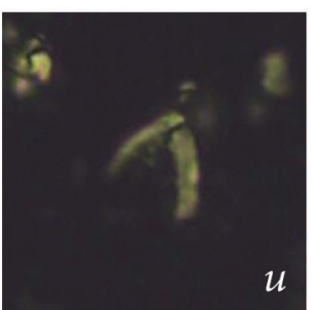

Ceratolithoides kamptneri

Kl-175.5 (XPL)
(GP)

(GP)

(PPL)

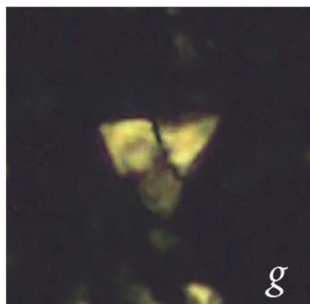

Uniplanarius trifidus Kl-73 (XPL)
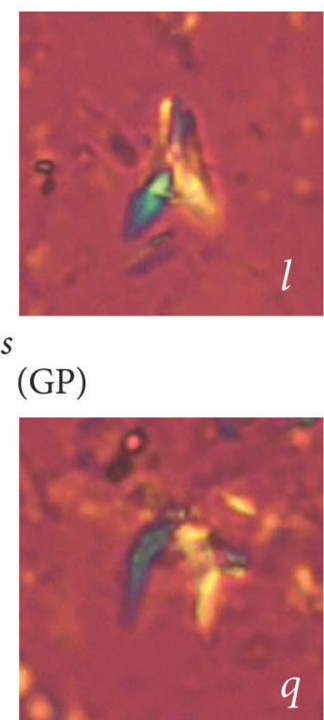

$q$

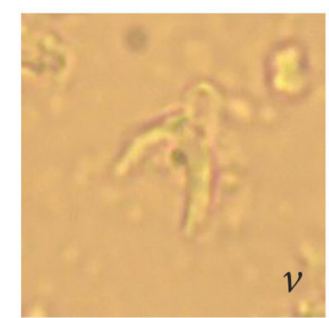

(PPL)

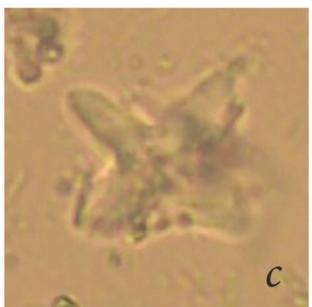

(PPL)

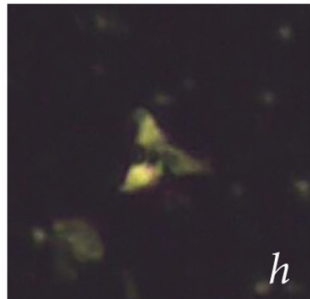

Uniplanarius trifidus K1-77.5 (XPL)

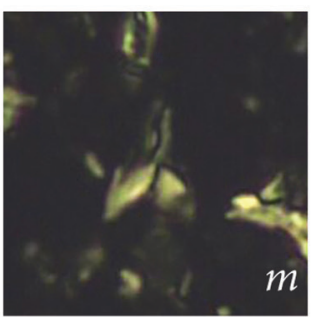

Ceratolithoides aculeus Ceratolithoides aculeus K1-73 (XPL)

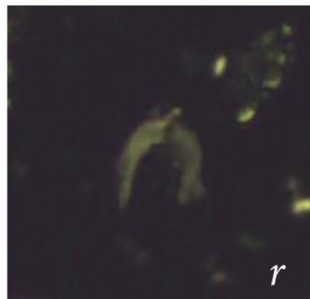

Cerato. kamptneri K1-175 (XPL)

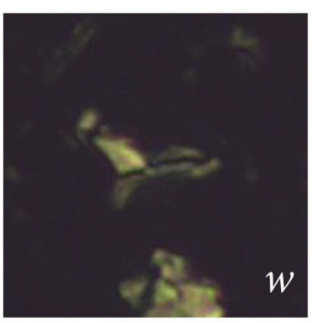

Cerato. sesquipedalis K1-77 (XPL)

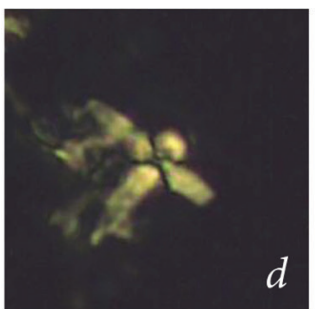

Uniplanarius sissinghii Uniplanarius trifidus K1-96.5 (XPL)

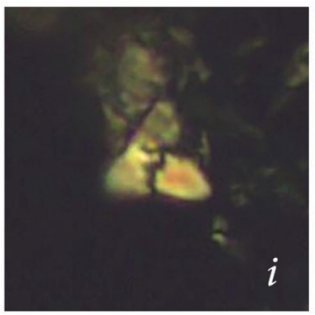

Uniplanarius trifidus Kl-96.5 (XPL)

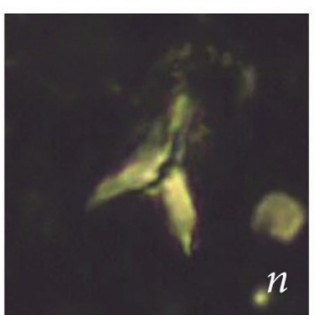

Kl-123 (XPL)

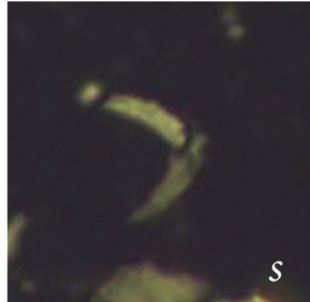

Cerato. kamptneri Kl-175 (XPL)

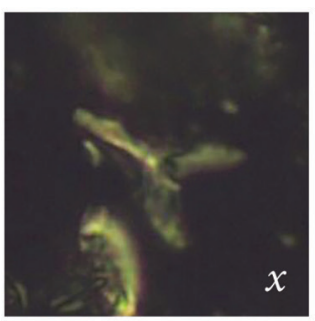

Ceratolithoides sesquipedalis

Kl-123 (XPL) (XPL)

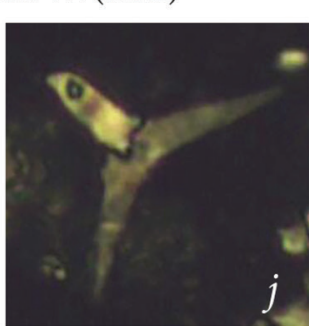

Uniplanarius trifidus Kl-96.5 (XPL)

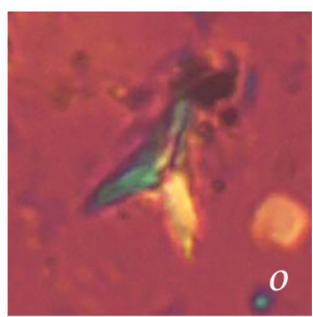

(GP)

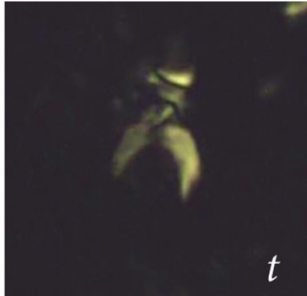

Cerato. kamptneri Kl-175 (XPL)

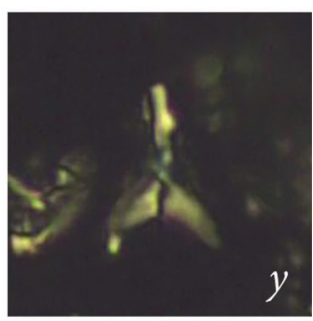

$5 \mu \mathrm{m}$

Fig. 18. Calcareous nannofossils from section Kladorub (nannoliths - Polycyclolithaceae, Ceratolithoides). Scale bar applies to all images. Abbreviations: XPL - cross-polarized light; PPL - plane-polarized light; GP - gypsum plate. 
poorly preserved, which is why these occurrences are likely to be the result of reworking. The latter assumption is further supported by the presence of slump structures in the upper half of the studied succession. Therefore, the boundary between zones UC15 and UC16 is placed at $38 \mathrm{~m}$. Two metres above the boundary, top Amphizygus brooksii (Fig. 4h) was detected.

Top Uniplanarius trifidus (Fig. 18e-j) was noted in sample Kl-116 (at $56 \mathrm{~m}$ ), followed by base Prediscosphaera mgayae (at 58 m; Fig. 11f, g), top Broinsonia parca subsp. parca (at $61 \mathrm{~m}$; Fig. 13a-e) and top Broinsonia parca subsp. constricta (at $62.5 \mathrm{~m}$; Fig. 13f). The latter event defines top UC16/base UC17. Recently, Thibault (2016a) commented that top Uniplanarius trifidus appears to be a highly useful biostratigraphic marker for the Campanian/ Maastrichtian boundary in the Tethyan realm and used it to subdivide Burnett's (1998) zone UC16 into two subzones: $\mathrm{UC} 16 \mathrm{a}^{\mathrm{TP}}$ and $\mathrm{UC} 16 \mathrm{~b}^{\mathrm{TP}}$. This new subdivision for the Tethyan province has been applied in the present study. Thus, both base Prediscosphaera mgayae and top Broinsonia parca subsp. parca fall within UC16b ${ }^{\mathrm{TP}}$.

The upper boundary of UC17 was drawn at $71.5 \mathrm{~m}$ by the last occurrence of Tranolithus orionatus (Fig. $5 w-y$ ). In the frame of this biostratigraphic unit, three nannofossil events were detected: (1) the concomitant tops of Uniplanarius gothicus (Fig. 17s-w) and Uniplanarius sissinghii (Figs $17 x, y, 18 a-d) 50 \mathrm{~cm}$ above base UC17; (2) top Acuturris scotus (Fig. $13 u$ ) at $70 \mathrm{~m}$. Top Reinhardtites levis (Fig. 5h-l), which defines the upper boundary of UC18, was noted at $75.5 \mathrm{~m}$.

The first reliably identifiable specimens of Lithraphidites quadratus (Fig. 15a-j), the base of which marks top UC19/base UC20, was observed in sample Kl-156 (at $96 \mathrm{~m}$ ). The interval between top Reinhardtites levis and base Lithraphidites quadratus (i.e., zone UC19) contains the following five nannofossil events (from oldest to youngest):

1) top Lucianorhabdus cayeuxii (Fig. 14a) at 85.5. m;

2) top Zeugrhabdotus bicrescenticus (Fig. 6a, b) at $87 \mathrm{~m}$;

3) base Lithraphidites praequadratus (Fig. 15fj) at $92.5 \mathrm{~m}$;

4) base Arkhangelskiella maastrichtiensis (Fig. $12 v-y)$ at $94 \mathrm{~m}$;

5) base Nephrolithus frequens (Fig. $9 p-r$ ) at $94.5 \mathrm{~m}$.

The consecutive bases of Micula murus (Fig. 16 $k-t$ ), Ceratolithoides kamptneri (Fig. 18p-v) and Micula prinsii (Figs $16 u-y, 17 a-g$ ) define the bases of subzones UC20b ${ }^{\mathrm{TP}}$, UC20c $\mathrm{c}^{\mathrm{TP}}$ and $\mathrm{UC} 20 \mathrm{~d}^{\mathrm{TP}}$ at
108 m, $109 \mathrm{~m}$ and $113 \mathrm{~m}$, respectively. The last consistently occurring specimens of Calculites obscurus (Fig. 13v,w) and Prediscosphaera mgayae were noted within UC20a ${ }^{\mathrm{TP}}$ in samples Kl-159 (at $99 \mathrm{~m}$ ) and Kl-160.5 (at $100.5 \mathrm{~m}$ ). However, it is uncertain whether these reflect their true tops or not, since they were observed in samples taken immediately above a 3-m interval of slumped sediments, where reworking is apparent. Base Cribrosphaerella daniae was detected in subzone $\mathrm{UC}^{20} \mathrm{c}^{\mathrm{TP}}$ at $111 \mathrm{~m}$.

In the uppermost $2 \mathrm{~m}$ of the studied succession, Cyclagelosphaera alta (Fig. 12a-e) is present, which, in the absence of Cruciplacolithus intermedius, indicates Martini's (1971) lowermost Danian zone NP1. Due to recent massive landslide processes, the Cretaceous/Palaeogene boundary falls within a 6.5-m interval of non-exposure, buried under a thick layer of soil, and efforts to uncover it have so far been futile. Nevertheless, attempts at removing the soil cover are in progress so that the Cretaceous/ Palaeogene boundary can be better constrained.

\section{DISCUSSION}

\section{Correlation with local nannofossil zonation schemes for the upper Campanian- Maastrichtian interval}

A complete nannofossil zonation scheme for the Upper Cretaceous of Bulgaria was developed by Sinnyovsky (2007, 2013a, 2015). In the upper Campanian (pars.)-Maastrichtian interval, Sinnyovsky (2013a, 2015) recognized a total of five zones and six subzones (Fig. 19), mostly based on nannofossil datums also employed by the CC zonation of Sissingh (1977), as modified by Perch-Nielsen (1985), and the UC scheme of Burnett (1998). One of these events, base Lithraphidites praequadratus, used by Sinnyovsky (2007, 2013a, 2015) to demarcate the lower boundary of his Lithraphidites praequadratus Subzone in the uppermost lower Maastrichtian, is strikingly unreliable because it has been demonstrated to be highly diachronous within the Campanian-Maastrichtian interval in both Bulgaria and elsewhere. Ivanov et al. (2007) reported this species from the upper Campanian in the Kraishte Zone (SW Bulgaria) in association with Eiffellithus eximius and Uniplanarius trifidus. Pérez-Rodríquez et al. (2012, Table 3) documented a reasonably consistent stratigraphical range for Lithraphidites praequadratus from the upper Campanian $\mathrm{UC} 15 \mathrm{e}^{\mathrm{TP}}$ to the upper upper Maastrichtian UC20d ${ }^{\mathrm{TP}}$ at Zumaia (northern Spain). In the Danish Basin, Thibault et al. (2012a) observed its base in the lowermost 


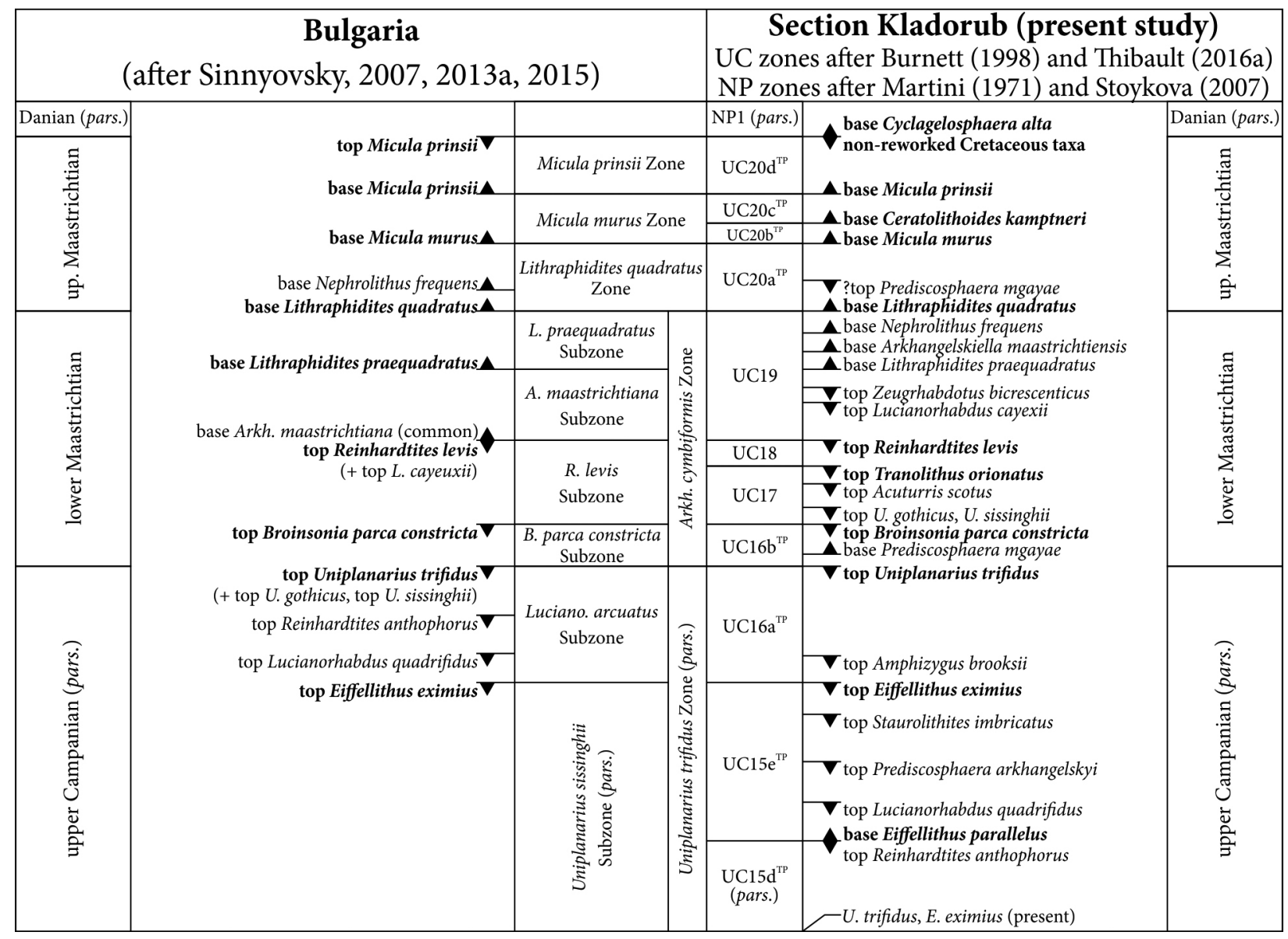

Fig. 19. Comparison of nannofossil datums at Kladorub (this study) with the local zonation scheme for Bulgaria of Sinnyovsky (2007, 2013a, 2015).

Maastrichtian, within subzone UC16 $\mathrm{d}^{\mathrm{BP}}$, whereas, in the Indian Ocean (ODP Hole 762C), it was detected in the Maastrichtian UC19 (Thibault et al., 2012b). In the present study, the first reliably identifiable specimens of Lithraphidites praequadratus were noted in UC19. This seems to be a real signal since this taxon is characterized by a consistent stratigraphical range up-section and rapidly becomes a considerable constituent of the upper Maastrichtian assemblages, taxonomic concepts were meticulously applied, and there are no deleterious changes in preservation to explain any possible loss of Lithraphidites praequadratus in lower samples. Interestingly, according to Sinnyovsky (2007, 2013a, 2015), base Lithraphidites praequadratus also post-dates base common Arkhangelskiella maastrichtiensis, which is not the case in section Kladorub. In the present study, the first rare representatives of Arkhangelskiella maastrichtiensis (sensu Burnett, 1997) were noted $1.5 \mathrm{~m}$ above base Lithraphidites praequadratus, in the upper part of UC19, and the former taxon does not become com- mon until mid-UC20a ${ }^{\mathrm{TP}}$. At Zumaia, base Arkhangelskiella maastrichtiensis has also been recorded within UC19, well above base Lithraphidites praequadratus (Pérez-Rodríguez et al., 2012). In the Danish Basin, Thibault et al. (2012a) also reported the first occurrence of Arkhangelskiella cymbiformis var. W (sensu Thibault, 2010a; equivalent to Arkhangelskiella maastrichtiensis) in the upper part of UC19 and above base Lithraphidites praequadratus.

Regarding secondary nannofossil events, many discrepancies between section Kladorub and Sinnyovsky's (2007, 2013a, 2015) zonation were found (see Fig. 19). For instance, (1) the tops of Reinhardtites anthophorus and Lucianorhabdus quadrifidus predate top Eiffellithus eximius; (2) the tops of Uniplanarius gothicus and Uniplanarius sissinghii not only do not coincide with top Uniplanarius trifidus but even post-date top Broinsonia parca subsp. constricta; (3) top Lucianorhabdus cayeuxii was detected above top Reinhardtites levis; (4) base Nephrolithus frequens predates base Lithraphidites 
quadratus; (5) top Zeugrhabdotus bicrescenticus was noted within zone UC19 in Kladorub, whereas Sinnyovsky (2007, 2013a, 2015) described it as consistently present to end-Cretaceous. Of the secondary datums recognized in the present study, the tops of Acuturris scotus (in UC17) and Zeugrhabdotus bicrescenticus (in UC19) are worthy of note. Recently, Pérez-Rodríguez et al. (2012) opined that these events may prove to have wide low-latitude stratigraphic and correlative value. In the tropical Indian Ocean, top Acuturris scotus approximates the top of Tranolithus orionatus (Lees, 2002). At Zumaia, top Acuturris scotus lies immediately above top Tranolithus orionatus (Pérez-Rodríguez et al., 2012) and, in Kladorub, the former biohorizon was also detected in very close proximity to the latter, albeit below. In the tropical Pacific Ocean, top Zeugrhabdotus bicrescenticus lies above top Tranolithus orionatus (Lees and Bown, 2005) and, in the tropical South Atlantic, it falls in the lower part of UC19 right above top Reinhardtites levis (Thibault, 2016a). The same observation has been made in the Zagros Basin (Iran) in the eastern Tethys (Razmjooei et al., 2018). However, these bioevents are not well documented in Bulgarian sections and further investigations are needed to test their stratigraphic utility.

\section{The Campanian/Maastrichtian and lower/upper Maastrichtian boundaries in section Kladorub}

\section{The Campanian/Maastrichtian boundary}

The Campanian/Maastrichtian boundary is nowadays officially defined as lying at $115.2 \mathrm{~m}$ on platform IV of the geological site at Tercis les Bains (Landes, France), the GSSP having been ratified in 2001 (Odin and Lamaurelle, 2001). This level corresponds to the arithmetic mean of 12 biohorizons including ammonites, dinoflagellate cysts, planktonic and benthic foraminifera, inoceramid bivalves and calcareous nannofossils, the first occurrence of ammonite Pachydiscus neubergicus being the primary criterion. In nannofossil terms, the Campanian/ Maastrichtian boundary at Tercis has been shown to lie between the tops of Eiffellithus eximius (below) and Uniplanarius trifidus (above) (Gardin et al., 2001), the latter having been chosen as one of the aforementioned 12 biohorizons (Odin and Lamaurelle, 2001). Prior to this ratification, the boundary was correlated with top Broinsonia parca subsp. constricta in the UC zonation of Burnett (1998). Pérez-Rodríguez et al. (2012) also heeded that there is evidence of top Uniplanarius trifidus lying above top Broinsonia parca subsp. constricta, as exemplified by the CC scheme of Sissingh (1977), as modified by Perch-Nielsen (1985), and Burnett's (1998) UC zonation, and so they deemed the use of top Uniplanarius trifidus as an indicator for the Campanian/Maastrichtian boundary outside of Tercis questionable. However, based on comparisons of four of the main reference sites for the Campanian/ Maastrichtian boundary (i.e., Tercis les Bains, Gubbio, DSDP Site 525a and ODP Site 762c), Thibault (2016a) demonstrated that top Uniplanarius trifidus is an excellent biostratigraphic marker for the Campanian/Maastrichtian boundary in the Tethyan realm. Due to the lack of proper chronostratigraphic framework for section Kladorub, this event has been used to draw the boundary in the present study (at $56 \mathrm{~m}$ ).

\section{The lower/upper Maastrichtian boundary}

There is still no formal agreement for the placement of a lower/upper Maastrichtian boundary. Paul and Lamolda (2007) highlighted base Lithraphidites quadratus as a potential marker for the lower/upper Maastrichtian boundary at Zumaia. Since Zumaia is a candidate substage-boundary stratotype for the lower/upper Maastrichtian boundary, Pérez-Rodríguez et al. (2012) proposed the following defining criteria: (1) the C31r/C31n magnetic reversal; (2) the base of calcareous nannofossil Lithraphidites quadratus; and (3) the base of the planktonic foraminifer Racemiguembelina fructicosa. In regards to calcareous nannoplankton, they also commented that Lithraphidites quadratus has a wide geographical distribution and the proximity of its base to the base of C31n makes it an acceptable candidate for defining the substage boundary, at least at low to mid latitudes. At Gubbio, the first occurrence of Lithraphidites quadratus has also been shown to lie very close to the base of Chron C31n and has been used to draw the lower/upper Maastrichtian boundary (Gardin et al., 2012). The same nannofossil biohorizon has also been used as a marker for the base of the upper Maastrichtian in the Danish Basin (Thibault et al., 2012a, b) and in the Zagros Basin, Iran (Razmjooei et al., 2014, 2018). Thus, again due to the lack of proper chronostratigraphic framework for section Kladorub, the lower/upper Maastrichtian boundary in the present study has been placed at the base of Lithraphidites quadratus at $96 \mathrm{~m}$.

\section{CONCLUSIONS}

New calcareous nannofossil data from the upper Campanian-Maastrichtian portion of the Kladorub 
Formation in NW Bulgaria have allowed detailed biostratigraphic framework to be erected. This zonation offers higher stratigraphic resolution than previously used local zonation schemes. Since it relies on well-established, traceable on a global scale bioevents, it facilitates further correlation with coeval sedimentary successions in other parts of the world, both in the Tethyan and the Boreal realm. Due to the lack of proper chronostratigraphic framework for the Kladorub Formation, top Uniplanarius trifidus and base Lithraphidites quadratus have been used to approximate the Campanian/Maastrichtian and lower/upper Maastrichtian boundaries, respectively, based on published data from elsewhere. The uppermost Maastrichtian-lowermost Danian strata are completely buried under a thick soil cover as a result of recent landslide processes, and thus further investigations are needed to place precisely the position of the Cretaceous/Palaeogene boundary in the section.

\section{Acknowledgements}

Warm thanks are expressed to Prof. Kristalina Stoykova for long, passionate conversations on nannofossil taxonomy and biostratigraphy, and to her and Dr Valeri Sachanski for fruitful discussions and encouragement. Two anonymous reviewers are greatly acknowledged for much-appreciated critical readings of the manuscript, comments and invaluable suggestions, which improved the quality of the original text and graphics. This paper is a contribution to the Project No DFNP-17-78/28.07.2017, funded by the Bulgarian Academy of Sciences.

\section{APPENDIX}

Table A1. Calcareous nannofossil stratigraphic rangechart, section Kladorub. (This table is available online in an over-sized format at https://www.geologica-balcanica.eu/sites/default/files/supplementary/05_Granchovski_Geol_Balc_48-1_2019_Appendix.pdf.)

\section{REFERENCES}

Angelov, V., Antonov, M., Gerdzhikov, S., Klimov, I., Petrov, P., Kiselinov, H., Dobrev, G. 2006. Explanatory notes for the geologic map of Bulgaria in scale 1:50 000, Rakovitsa map sheet. Bulgarian National Geological Survey, Sofia, $69 \mathrm{pp}$.

Angelov, V., Dobrev, G. 2006a. Rabisha Formation. In: Angelov, V., Khrischev, K. (Eds), Explanatory notes for the geologic map of Bulgaria in scale 1:50 000, Dimovo map sheet. Bulgarian National Geological Survey, Sofia, 39-40.

Angelov, V., Dobrev, G. 2006b. Kula Formation. In: Angelov, V., Khrischev, K. (Eds), Explanatory notes for the geologic map of Bulgaria in scale 1:50 000, Dimovo map sheet. Bulgarian National Geological Survey, Sofia, 40-42.

Angelov, V., Dobrev, G. 2006c. Kladorub Formation. In: Angelov, V., Khrischev, K. (Eds), Explanatory notes for the geologic map of Bulgaria in scale 1:50 000, Dimovo map sheet. Bulgarian National Geological Survey, Sofia, 42-44.

Angelov, V., Dobrev, G. 2006d. Rabisha Formation. In: Angelov, V., Khrischev, K. (Eds), Explanatory notes for the geologic map of Bulgaria in scale 1:50 000, Ruzhintsi map sheet. Bulgarian National Geological Survey, Sofia, 60-61.

Angelov, V., Dobrev, G. 2006e. Kula Formation. In: Angelov, V., Khrischev, K. (Eds), Explanatory notes for the geologic map of Bulgaria in scale 1:50 000, Ruzhintsi map sheet. Bulgarian National Geological Survey, Sofia, 61-62.

Bown, P.R., Young, J.R. 1998. Techniques. In: Bown, P.R. (Ed.), Calcareous nannofossil biostratigraphy. British Micropalaeontological Society Publication Series. Chapman \& Hall/Kluwer Academic Publishers, London, 16-28.

Burnett, J.A. 1997. New species and new combinations of Cretaceous nannofossils, and a note on the origin of Petrarhabdus (Deflandre) Wind \& Wise. Journal of Nannoplankton Research 19 (2), 133-146.

Burnett, J.A. 1998. Upper Cretaceous. In: Bown, P.R. (Ed.), Calcareous nannofossil biostratigraphy. British Micro- palaeontological Society Publication Series. Chapman \& Hall/Kluwer Academic Publishers, London, 132-199.

Cheshitev, G., Kanchev, I., Valkov, V., Marinova, R., Shilyafova, Zh., Ruseva, M., Iliev, K. 1989. Geological map of Bulgaria in scale 1:500 000. Committee of Geology, Sofia.

Dabovski, H. 2009. Upper Cretaceous geology. Introduction. In: Zagorchev, I., Dabovski, H., Nikolov, T. (Eds), Geology of Bulgaria. Vol. II. Mesozoic geology. "Prof. M. Drinov" Academic Press, Sofia, 305-307 (in Bulgarian, with English abstract).

Dabovski, H., Kamenov, B., Sinnyovsky, D., Vasilev, E., Dimitrova, E., Bayraktarov, I. 2009. Upper Cretaceous geology. In: Zagorchev, I., Dabovski, Ch., Nikolov, T. (Eds), Geology of Bulgaria. Vol. II. Mesozoic geology. "Prof. Marin Drinov" Academic Press, Sofia, 303-589 (in Bulgarian, with English summary).

Dabovski, H., Zagorchev, I. 2009. Introduction: Mesozoic evolution and Alpine structure. Alpine structure. In: Zagorchev, I., Dabovski, H., Nikolov, T. (Eds), Geology of Bulgaria. Vol. II. Mesozoic geology. "Prof. M. Drinov" Academic Press, Sofia, 30-37 (in Bulgarian, with English abstract).

Decheva, A., Filipov, L., Haydutov, I., Tzankov, Tz., Kojumdgieva, E., Popov, N. 1995. Explanatory notes for the geologic map of the Republic of Bulgaria in scale 1:100 000, Zaechar and Bor map sheets. Avers ST, Sofia, 43 pp. (in Bulgarian, with English abstract).

Dobrev, G. 2006. Rabisha Formation. In: Angelov, V., Khrischev, K. (Eds), Explanatory notes for the geologic map of Bulgaria in scale 1:50 000, Zaechar and Bor map sheets. Bulgarian National Geological Survey, Sofia, 16-17.

Dobrev, G., Angelov, V. 2006. Kula Formation. In: Angelov, V., Khrischev, K. (Eds), Explanatory notes for the geologic map of Bulgaria in scale 1:50 000, Ruzhintsi map sheet. Bulgarian National Geological Survey, Sofia, 17-19. 
Filipov, L., Decheva, A., Kojumdgieva, E., Popov, N., Tzankov, Tz., Haydutov, I. 1995. Explanatory notes for the geologic map of the Republic of Bulgaria in scale 1:100 000, Vidin map sheet. Avers ST, Sofia, 60 pp. (in Bulgarian, with English abstract).

Gardin, S., Galbrun, B., Thibault, N., Coccioni, R., PremoliSilva, I. 2012. Biomagnetochronology for the upper Campanian-Maastrichtian from the Gubbio area, Italy: new results from the Contessa Highway and Bottaccione sections. Newsletters on Stratigraphy 45, 75-103.

Gardin, S., Odin, G.S., Bonnemaison, M., Melinte, M., Monechi, S., von Salis, K. 2001b. Results of the cooperative study on the calcareous nannofossils across the Campanian-Maastrichtian boundary at Tercis les Bains (Landes, France). In: Odin, G.S. (Ed.), The Campanian-Maastrichtian Stage Boundary. Characterization at Tercis les Bains (France) and Correlation with Europe and Other Continents. Developments in Palaeontology and Stratigraphy 19, 293-309.

Granchovski, G. 2012. Calcareous nannofossils from the type section for the Rabisha Formation (NW Bulgaria). Bulgarian Geological Society, National Conference with international participation "Geosciences 2012", Abstracts, 81-82.

Granchovski, G. 2013. New data on the age of the Rabisha Formation from borehole Belo Pole 1 (NW Bulgaria). Bulgarian Geological Society, National Conference with international participation “Geosciences 2013”, Abstracts, 71-72 (in Bulgarian).

Granchovski, G. 2015. Uppermost Albian-lower Cenomanian calcareous nannofossils from the Shishentsi-1 and Makresh-1 boreholes, Kula tectonic unit (NW Bulgaria). Geologica Balcanica 44 (1-3), 25-38.

Granchovski, G. 2017. First calcareous nannofossil data on the Turonian in the Kula Formation (NW Bulgaria). Comptes rendus de l'Académie bulgare des Sciences 70 (3), 399-404.

Harkovaska, A., Cvetkov, A., Pecskay, Z., Stoykova, K., Kamenov, B., Ivanov, D. 1999. Late Cretaceous (Timok Volcanics) epiclastics in the Upper Cretaceous of the Northwestern Bulgaria. Bulletin de l'Académie serbe des Sciences et Arts, classe des Sciences mathématiques et naturelles, Sciences naturelles 39; 19-35.

Haydoutov, I., Yanev, S., Tronkov, D., Sapunov, I., Tchoumatchenko, P., Tzankov, Tz., Popov, N., Dimitrova, R., Nikolov, T., Aladjova-Khrischeva, K., Chunev, D., Filipov, L. 1995. Explanatory notes for the geologic map of Bulgaria in scale 1:100 000, Belogradchik map sheet. Avers ST, Sofia, 42 pp. (in Bulgarian, with English abstract).

Ivanov, M., Stoykova, K., Zagorchev, I., Goranov, E. 2007. Lithostratigraphy of the Upper Cretaceous Series in a part of the Krayshte area (Southwest Bulgaria). Geologica Balcanica 36 (3-4), 69-80.

Ivanov, Ž. 1983. Aperçu général sur l'évolution géologique et structurale des Balkanides. In: Ivanov, Ž., Nikolov, T. (Eds), Guide de l'excursion géologique de la Société Géologique de France en Bulgarie, Sofia, 3-26.

Ivanov, Ž. 1998. Tectonics of Bulgaria. Professorship thesis, Sofia University “St Kliment Ohridski”, 579 pp. (in Bulgarian, unpublished).

Ivanov, Ž. 2017. Tectonics of Bulgaria. Sofia University Press, Sofia, 331 pp. (in Bulgarian, with English summary).

Lees, J.A. 2002. Calcareous nannofossil biostratigraphy illustrates palaeoclimate change in the Late Cretaceous Indian Ocean. Cretaceous Research 23 (5), 537-634.

Lees, J.A. 2007. New and rarely reported calcareous nannofossils from the Late Cretaceous of coastal Tanzania: outcrop samples and Tanzania Drilling Project Sites 5, 9 and 15. Journal of Nannoplankton Research 29 (1), 39-65.
Lees, J.A., Bown, P.R. 2005. Upper Cretaceous calcareous nannofossil biostratigraphy, ODP Leg 198 (Shatsky Rise, northwest Pacific Ocean). Proceedings of the Ocean Drilling Program, Scientific Results 198, 1-60 (http://www-odp. tamu.edu/publications/198_SR/VOLUME/CHAPTERS/ 114.PDF).

Martini, E. 1971. Standard Tertiary and Quaternary calcareous nannoplankton zonation. In: Farinacci, A. (Ed.), Proceedings of the Second Planktonic Conference, Roma, 1970, Tecnoscienza, 739-785.

Monova, D., Ivanov, V. 1982. Report on the results of the Makresh-1 borehole conducted in 1979-1980. Ministry of Economics, National Geological Fund, 187 pp. (in Bulgarian, unpublished).

Nachev, I., Sultanov, Y. 1991. Sedimentology of the Upper Cretaceous in Bulgaria. Bulgarian Academy of Sciences Academic Press, Sofia, 80 pp. (in Bulgarian).

Nachev, I., Yanev, S. 1980. Sedimentary geocomplexes in Bulgaria. “Nauka i izkustvo” Publishing House, Sofia, 203 pp. (in Bulgarian).

Nikolov, T., Ruskova, N. 1993. The Lower Cretaceous in the wells of Northwestern Bulgaria. Geologica Balcanica 23 (5), 47-67.

Odin, G.S., Lamaurelle, M.A. 2001. The global Campanian/ Maastrichtian stage boundary. Episodes 24, 229-238.

Paul, C.R.C., Lamolda, M.A. 2007. Carbon and oxygen stable isotopes in the Maastrichtian of the Basque Country, N. Spain. Cretaceous Research 28, 812-820.

Perch-Nielsen, K. 1985. Mesozoic calcareous nannofossils. In: Bolli, H.M., Saunders, J.B., Perch-Nielsen, K. (Eds), Plankton stratigraphy. Cambridge University Press, Cambridge, 329-426.

Pérez-Rodríguez, I., Lees, J.A., Larrasoaña, J.C., Arz, J.A., Arenillas, I. 2012. Planktonic foraminiferal and calcareous nannofossil biostratigraphy and magnetostratigraphy of the uppermost Campanian and Maastrichtian at Zumaia, northern Spain. Cretaceous Research 37, 100-126.

Peycheva, E., Parvanova, A. 1989. Report on the geologic results from the exploratory drilling conducted near Belo Pole in 1979-1980. Ministry of Economics, National Geological Fund, 202 pp. (in Bulgarian, unpublished).

Razmjooei, M.J., Thibault, N., Kani, A., Dinarès-Turell, J., Pucéat, E., Shahriari, S., Radmacher, W., Jamali, A.M., Ullmann, C.V., Voigt, S., Cocquerez, T. 2018. Integrated bio- and carbon-isotope stratigraphy of the Upper Cretaceous Gurpi Formation (Iran): A new reference for the eastern Tethys and its implications for large-scale correlation of stage boundaries. Cretaceous Research 91, 312-340.

Razmjooei, M.J., Thibault, N., Kani, A., Mahanipour, A., Boussaha, M., Korte, C. 2014. Coniacian-Maastrichtian calcareous nannofossil biostratigraphy and carbon-isotope stratigraphy in the Zagros Basin (Iran): consequences for the correlation of Late Cretaceous Stage Boundaries between the Tethyan and Boreal realms. Newsletters on Stratigraphy 47 (2), 183-209.

Shamrock, J.L., Watkins, D.K. 2009. Evolution of the calcareous nannofossil genus Eiffellithus and its biostratigraphic significance. Cretaceous Research 30, 1083-1102.

Sinnyovsky, D. 2003. Five protected outcrops of the Cretaceous/Tertiary boundary in Bulgaria. Annual of the University of Mining and Geology 46 (1), 141-147 (in Bulgarian).

Sinnyovsky, D. 2004. Calcareous nannoplankton biostratigraphy of the Carpathian type Upper Cretaceous-Paleocene deposits near Kladorub Village, Vidin District. Annual of the University of Mining and Geology 47, 139-145.

Sinnyovsky, D. 2007. Biostratigraphy of the Upper Cretaceous of Bulgaria based on calcareous nannoplankton. DSc the- 
sis, University of Mining and Geology "St Ivan Rilski”, Sofia, 268 pp. (in Bulgarian, unpublished).

Sinnyovsky, D. 2009. Upper Cretaceous geology. Carpathian type Upper Cretaceous. In: Zagorchev, I., Dabovski, H., Nikolov, T. (Eds), Geology of Bulgaria. Vol. II. Mesozoic geology. "Prof. M. Drinov" Academic Press, Sofia, 389394 (in Bulgarian, with English abstract).

Sinnyovsky, D. 2011. The Ruzhintsi Formation, a unique facies in the Carpathian type Upper Cretaceous and Paleocene in NW Bulgaria. Bulgarian Geological Society, National Conference with International Participation "Geosciences 2013", Abstracts, 91-92.

Sinnyovsky, D. 2013a. Upper Cretaceous calcareous nannoplankton biostratigraphy in Bulgaria. "St Ivan Rilski” Publishing House, Sofia, 80 pp.

Sinnyovsky, D. 2013b. Outcrops of the Mirkovo and Zavala Formations in NW Bulgaria. Bulgarian Geological Society, National Conference with International Participation “Geosciences 2013”, Abstracts, 77-78.

Sinnyovsky, D. 2015. Upper Cretaceous calcareous nannoplankton biostratigraphy in Bulgaria. LAP Lambert Academic Publishing, Saarbrücken, 152 pp.

Sinnyovsky, D., Pavlishina, P. 2014. Nannoplankton and palynological evidence for the Albian-Cenomanian boundary in Northwest Bulgaria. Comptes rendus de l'Académie bulgare des Sciences 53 (11), 41-44.

Sinnyovsky, D., Petrov, G. 2000. Nannofossil evidences for Maastrichtian-Paleocene age of the Kladorub Formation in NW Bulgaria. Comptes rendus de l'Académie bulgare des Sciences 53 (11), 41-44.

Sinnyovsky, D., Valchev, B., Sinnyovska, D. 2002. Cretaceous/ Tertiary boundary in the Carpathian type Upper Cretaceous near the village of Kladorub, Vidin District. Annual of the University of Mining and Geology 45 (1), 1-5.

Sissingh, W. 1977. Biostratigraphy of the Cretaceous calcareous nannoplankton. Geologie en Mijnbouw 56 (1), 37-65.

Stoykova, K. 2007. Stratigraphy of the Paleocene and lower Eocene of North Bulgaria based on calcareous nannofossils. DSc thesis, Geological Institute "Str. Dimitrov", Bulgarian Academy of Sciences, Sofia, 339 pp. (in Bulgarian, unpublished).

Stoykova, K., Dinarès-Turell, J., Ivanov, M. 2006. Calcareous nannofossil record along the Paleocene-Eocene transition at Kladorub and Riben sections, Bulgaria. In: Climate and Biota of the Early Paleogene, Bilbao, 2006, Volume of Abstracts, p. 133.

Stoykova, K., Ivanov, M. 2005. First data on the presence of the Paleocene-Eocene Thermal Maximum in Bulgaria. Comptes rendus de l'Académie bulgare des Sciences 58 (3), 297-302.

Stoykova, K., Juranov, S., Ivanov, M. 2005. Calibration of calcareous nannofossils and planktonic foraminifers' zonation in the Lower and Middle Paleocene in Bulgaria. Geologica Balcanica 35 (1-2), 3-8.

Thibault, N. 2010a. Biometric analysis of the Arkhangelskiella group in the upper Campanian-Maastrichtian of the Stevns-1 borehole, Denmark: Taxonomic implications and evolutionary trends. Geobios 43, 639-652.

Thibault, N. 2010b. Calcareous nannofossils from the boreal Upper Campanian-Maastrichtian chalk of Denmark. Journal of Nannoplankton Research 31 (1), 39-56.

Thibault, N. 2016a. Calcareous nannofossil biostratigraphy and turnover dynamics in the late Campanian-Maastrichtian of the tropical South Atlantic. Revue de micropaléontologie 59, 57-69.

Thibault, N. 2016b. Reliability of calcareous nannofossil consistent last occurrences in the chalk: an example from the
Late Cretaceous of Denmark. New Mexico Museum of Natural History and Science Bulletin 71, 299-306.

Thibault, N., Harlou, R., Schovsbo, N., Schioler, P., Minoletti, F., Galbrun, B., Lauridsen, B.W., Sheldon, E., Stemmerik, L., Surlyk, F. 2012a. Upper Campanian-Maastrichtian nannofossil biostratigraphy and high-resolution carbon-isotope stratigraphy of the Danish Basin: Towards a standard $\delta^{13} \mathrm{C}$ curve for the Boreal Realm. Cretaceous Research 33, 72-90.

Thibault, N., Husson, D., Harlou, R., Gardin, S., Galbrun, B., Huret, E., Minoletti, F. 2012b. Astronomical calibration of upper Campanian-Maastrichtian carbon isotope events and calcareous plankton biostratigraphy in the Indian Ocean (ODP Hole 762C): Implication for the age of the Campanian-Maastrichtian boundary. Palaeogeography, Palaeoclimatology, Palaeoecology 337-338, 52-71.

Tzankov, Tz. 1961. Notes on the relations between the South Carpathians and the Balkanides in Northwest Bulgaria. Travaux sur la Géologie de Bulgarie, Série Stratigraphie et tectonique 3, 173-251 (in Bulgarian).

Tzankov, Tz. 1963a. The Maastrichtian near Ruzhintsi Village (Belogradchik District) and the question about the eastern limit of the South Carpathians in Bulgaria. Bulletin of the Geological Institute 12, 77-89.

Tzankov, Tz. 1963b. Stratigraphy of the Carpathian type Cretaceous in the area of Kula (Northwest Bulgaria). Bulletin de l'Association Géologique Carpato-Balkanique, V-ème Congrès, 1961, Bucarest, Communications Scientifiques, II-ème section: Stratigraphie 3 (2), 207-219 (in Russian).

Tzankov, Tz. 1964. Ammonites from the Maastrichtian near Kladorub Village, Belogradchik District (Northwest Bulgaria). Travaux sur la Géologie de Bulgarie, Série Palèontologie 6, 143-187 (in Bulgarian).

Tzankov, Tz. 1972. Jungalpidische Deformationen im KrajnaGebiet (Nordwestbulgarien). Geologie 21 (1), 24-60.

Tzankov, V. 1968. Upper Cretaceous. In: Tzankov, V., Spasov, H. (Eds), Stratigraphy of Bulgaria. "Nauka i izkustvo" Publishing House, Sofia, 253-294 (in Bulgarian).

Tzankov, V., Cheshitev, G., Dimitrova, N. 1963. Summary of the Upper Cretaceous stratigraphy in North Bulgaria. Bulletin de l'Association Géologique Carpato-Balkanique, V-ème Congrès, 1961, Bucarest, Communications Scientifiques, II-ème section: Stratigraphie 3 (2), 221-243 (in Russian).

Tzankov, V., Dimitrova, N., Stefanov, J., Vrablyanski, B. 1960. Stratigraphic studies on the Jurassic and Cretaceous Series in Northwest Bulgaria. Travaux sur la géologie de Bulgarie, série Stratigraphie et tectonique 1, 203-241 (in Bulgarian).

Valchev, B., Juranov, S. 2006. Planktic foraminiferal changes across the K/T boundary in the Carpathian type Upper Cretaceous and Paleocene near Kladorub Village, Vidin District (NW Bulgaria). Annual of the University of Geology and Mining 49 (1), 143-148.

Valkov, I. 1977. Report on the results of the Shishentsi-1 borehole drilled in 1974-1976. Ministry of Economy, National Geological Fund, 104 pp. (in Bulgarian, unpublished).

Vaptzarova, J. 1963. On the presence of the Danian Stage near the villages of Novo Selo and Koshava (Vidin District). Proceedings of the Bulgarian Geological Society 24 (1), 84-86 (in Bulgarian).

Vaptzarova, J. 1965a. Stratigraphy of the Upper Cretaceous in North and Northwest Bulgaria based on micropalaeontological data. Annual of the Head Office of Geology 15, 125-161 (in Bulgarian).

Vaptzarova, J. 1965b. Upper Cretaceous. In: Tzankov, V. (Ed.), Microfossil assemblages in Bulgaria. Principal Directorate of Geology, Sofia, 255-281 (in Bulgarian). 
Vaptzarova, J. 1974. Foraminifera from the Carpathian type Maastrichtian of Northwest Bulgaria. Proceedings of the Geological Institute, Series Palaeontology 23, 31-56 (in Bulgarian).

Vaptzarova, J. 1975a. Planktonic foraminifera and zonation of the Campanian in Northwest Bulgaria. Palaeontology, Stratigraphy and Lithology 1, 19-32 (in Bulgarian).

Vaptzarova, J. 1975b. Benthic foraminifera from the Campanian in Northwest Bulgaria. Palaeontology, Stratigraphy and Lithology 2, 57-72 (in Bulgarian).
Vaptzarova, J. 1976a. Zonation du Crétacé supérieur du type carpathique en Bulgarie du Nord-Ouest d'après les foraminifères planctoniques. Geologica Balcanica 6 (3), 93-109.

Vaptzarova, J. 1976b. Planktonic foraminifera from the Coniacian-Santonian of Northwest Bulgaria. Palaeontology, Stratigraphy and Lithology 4, 47-62 (in Bulgarian).

Vaptzarova, J. 1978. Foraminifera from the Coniacian-Santonian of Northwest Bulgaria. Palaeontology, Stratigraphy and Lithology 8, 55-68 (in Bulgarian). 